University of Wollongong

Research Online

Australian Institute for Innovative Materials -

Papers

Australian Institute for Innovative Materials

$1-1-2018$

Engineering of lithium-metal anodes towards a safe and stable battery

Lei Wang

Tianjin University

Ziyue Zhou

Tianjin University

Xiao Yan

Chinese Academy Of Sciences

Feng Hou

Tianjin University

Lei Wen

Chinese Academy Of Sciences

See next page for additional authors

Follow this and additional works at: https://ro.uow.edu.au/aiimpapers

Part of the Engineering Commons, and the Physical Sciences and Mathematics Commons

Research Online is the open access institutional repository for the University of Wollongong. For further information contact the UOW Library: research-pubs@uow.edu.au 


\title{
Engineering of lithium-metal anodes towards a safe and stable battery
}

\begin{abstract}
Currently, the state-of-the-art lithium-ion batteries (LIBs) are the most widely used energy storage devices and have brought a great impact on our daily life. However, even many strategies have been reported to improve the energy density, these LIBs still can not meet the rapidly growing demand from the many lately emerged devices. During the pursue of higher energy densities, lithium-metal batteries (LMBs) have been the most promising candidates of the next-generation energy storage devices. Unfortunately, the Li-metal anode usually induces severe safety concerns and inferior cycle performance, because of the dendrite growth, high reactivity, and infinite volume changes of Li metal. As a result, these problems limit the commercial application of LMBs and must be resolved prior to the practical deployment of LMBs. In this review, we will firstly discuss the failure mechanisms of Li-metal anodes and introduce latest characterization technologies to study dendritic Li formation. The advances to improve the safety and performance of Li metal anode through electrolyte modification, interfacial engineering, solid-state electrolyte incorporation, and host materials design will then be comprehensively summarized and discussed. Lastly, we will conclude by summarizing the challenges in the current research on LMBs and highlight the future perspectives as well. Through this review, we hope to present the latest developments of the Li metal anode materials for the readers, and also shed light on the possible solutions for the current issues in order to accelerate both fundamental research and practical deployment of the various LMBs.
\end{abstract}

\section{Disciplines}

Engineering | Physical Sciences and Mathematics

\section{Publication Details}

Wang, L., Zhou, Z., Yan, X., Hou, F., Wen, L., Luo, W., Liang, J. \& Dou, S. (2018). Engineering of lithium-metal anodes towards a safe and stable battery. Energy Storage Materials, 14 22-48.

\section{Authors}

Lei Wang, Ziyue Zhou, Xiao Yan, Feng Hou, Lei Wen, Wenbin Luo, Ji Liang, and Shi Xue Dou 


\title{
Engineering of Lithium-Metal Anodes towards a Safe and Stable Battery
}

\begin{abstract}
Currently, the state-of-the-art lithium-ion batteries (LIBs) are the most widely used energy storage devices and have brought a great impact on our daily life. However, even many strategies have been reported to improve the energy density, these LIBs still can not meet the rapidly growing demand from the many lately emerged devices. During the pursue of higher energy densities, lithium-metal batteries (LMBs) have been the most promising candidates of the next-generation energy storage devices. Unfortunately, the Li-metal anode usually induces severe safety concerns and inferior cycle performance, because of the dendrite growth, high reactivity, and infinite volume changes of Li metal. As a result, these problems limit the commercial application of LMBs and must be resolved prior to the practical deployment of LMBs. In this review, we will firstly discuss the failure mechanisms of Li-metal anodes and introduce latest characterization technologies to study dendritic Li formation. The advances to improve the safety and performance of Li metal anode through electrolyte modification, interfacial engineering, solid-state electrolyte incorporation, and host materials design will then be comprehensively summarized and discussed. Lastly, we will conclude by summarizing the challenges in the current research on LMBs and highlight the future perspectives as well. Through this review, we hope to present the latest developments of the Li metal anode materials for the readers, and also shed light on the possible solutions for the current issues in order to accelerate both fundamental research and practical deployment of the various LMBs.
\end{abstract}

Key Words: Li metal, surface protection, solid electrolyte, host materials, anode 


\section{Introduction}

Energy storage, especially with high density and low-cost, is always a hot spot in both research and industry communities. It is the fundamental requirement for the current and future energy strategies, for example, for the utilization of various clean energies (mostly harvested in the form of electricity), for the electrification of the transportation tools, as well as for the development of various smart devices. Lithium-ion batteries (LIBs) are one of the most successfully commercialized rechargeable batteries since the early 1990s, due to their high energy density and superior cycle stability, and have been widely deployed in various applications, including personal electronics and electric vehicles.[1]

For the LIBs, graphite is the most commonly used anode material, which can reversibly accommodate and release the $\mathrm{Li}$ ions that are extracted from the cathode materials $\left(\mathrm{LiMO}_{2}, \mathrm{M}=\mathrm{Ni}\right.$, Co, $\mathrm{Mn}$, etc., $\mathrm{LiFePO}_{4}$, and others) in its layered structure during charge/discharge processes (Figure 1a).[2] Unfortunately, even though many strategies, including modifying electrode materials and/or electrolytes, have been adopted to further improve the energy density of LIBs, they still can not meet the rapidly growing demands from the current devices, let alone the future ones, because these conventional battery materials are almost approaching their theoretical limitations.[2-4] Consequently, the next-generation energy storage materials/devices are in urgent needs of exploration. 


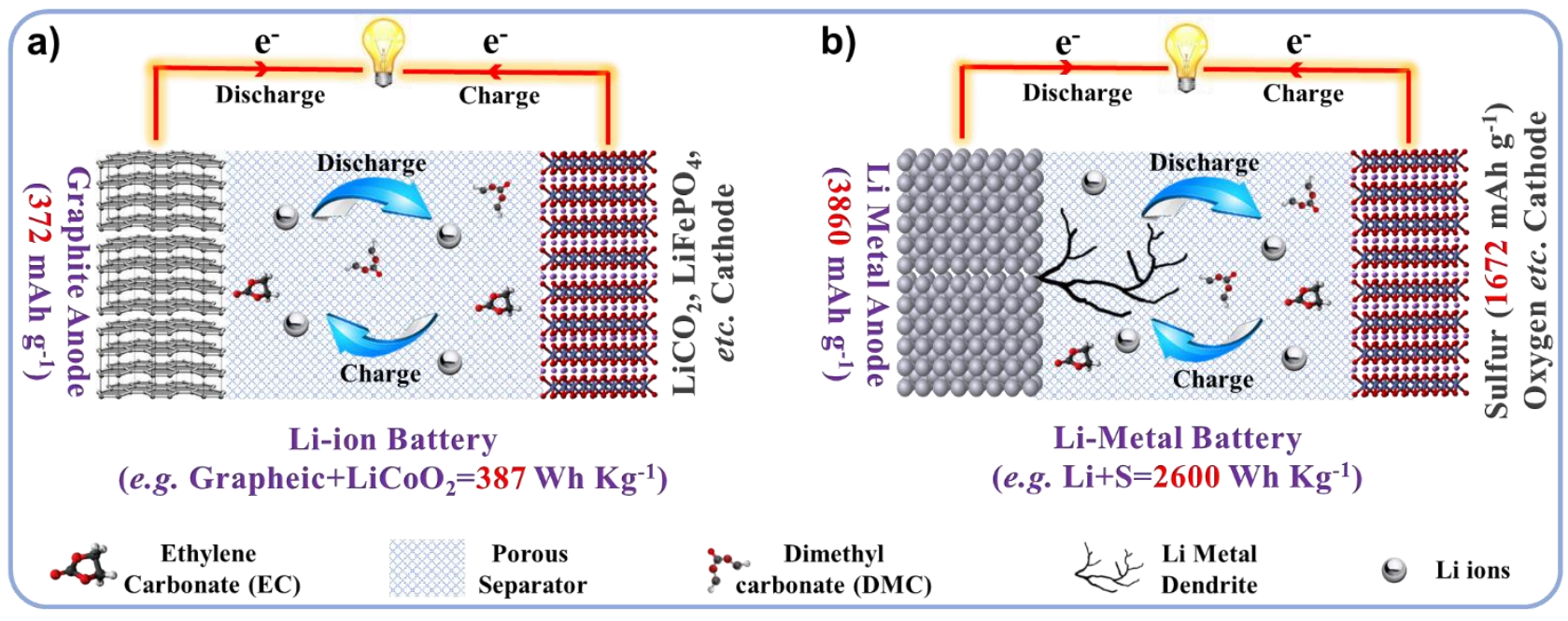

Figure 1. Operational principles of a) a Li-ion battery and b) a Li-metal battery.

With an extremely high capacity $\left(3860 \mathrm{mAh} \mathrm{g}^{-1}\right)$, low density $\left(0.59 \mathrm{~g} \mathrm{~cm}^{-3}\right)$, and very negative electrochemical potential $(-3.04 \mathrm{~V} v s$. the standard hydrogen electrode),[5] Li metal has been considered as an ideal anode material for the future Li-metal batteries (LMBs, Figure 1b).[6] By coupling it with the high capacity cathodes, the as-formed Li metal batteries (LMBs) possess exceptionally high energy densities compared to the traditional LIBs $\left(3500 \mathrm{Wh} \mathrm{kg}^{-1}\right.$ for $\mathrm{Li}-\mathrm{O}_{2}$ batteries and $2600 \mathrm{Wh} \mathrm{kg}^{-1}$ for Li-S batteries).[7-10]

In spite of these advantages, there are still some insurmountable obstacles that have greatly hindered the practical applications of these LMBs since their first emergence in 1970s.[11] Despite the different reaction mechanisms and respective cathode issues in these various LMBs (i.e. the sluggish oxygen reduction/evolution in $\mathrm{Li}-\mathrm{O}_{2}$ batteries or the irreversible polysulfide migration of in Li-S batteries), the challenges in the anode section of these battery systems are almost identical, which are closely related with the nature of Li metal and mainly include the dendrite growth, high reactivity, and infinite volume changes of metallic $\mathrm{Li}$ anode, which inevitably induces severe safety issues and inferior cycling performance/coulombic efficiency to the batteries.[12] 
Specifically, the growth of dendritic Li could cause the internal short circuit, generation of heat, or even explosion of battery. Moreover, it can also produce "dead Li" (i.e. the electrically detached Li dendrites),[13] which can compromise the coulombic efficiency and shorten the cycle life of LMBs. Meanwhile, Li metal is highly reactive, which will spontaneously react with the electrolytes, leading to uncontrollable solid electrolyte interface (SEI) formation on the surface of Li metal and resulting in the loss of active materials together with the increase of the cell impedance. Moreover, during the striping/plating of Li metal, the theoretically infinite volume change (i.e. ideally all the Li metal will be intercalated into the cathode upon fully discharge) would cause structural instability to the batteries, leading to a fast battery failure. Consequently, seeking effective strategies to stabilize the Li surface and bulky structure is essential to ameliorating the overall performance of LMBs for practical applications.

The nucleation and growth mechanism of Li dendrites have been continuously studied during the past 40 years. [14] Various approaches to stabilizing the Li metal surface have been proposed, which focus on protecting the Li surface via coating layers, optimizing the organic electrolytes, modifying the separators, and constructing novel host materials for Li-metal anodes.[4, 15, 16] Recently, to fundamentally resolve the thermodynamically unstable issue of the Li metal in conventional organic liquid electrolytes,[17] new battery configurations with solid-state electrolytes (SSEs) have also been adopted for the LMBs with the expectations to block Li dendrites, extend the cycle life of Li-metal batteries, as well as enhance the safety of the LMBs.[18, 19] 


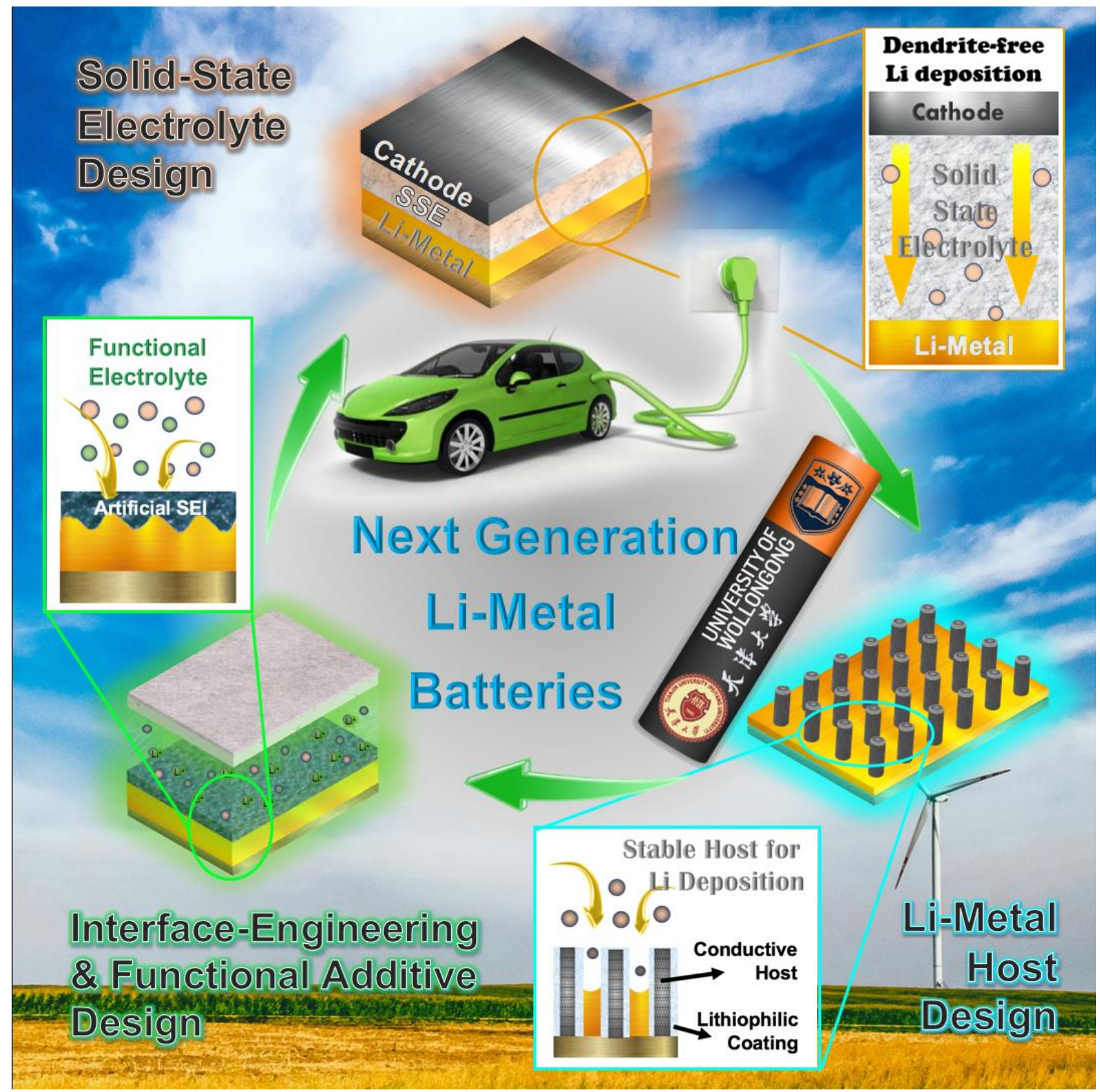

Scheme 1. Illustration of the strategies for constructing a safer Li metal anode.

In this review, we comprehensively summarize and discuss the recent scientific and technological strategies to construct the next generation Li-metal anodes for LMBs with higher energy density and better safety (Scheme 1). Firstly, we will firstly analyze the failure mechanisms of Li metal anodes and the fundamental principles for Li metal surface stabilization, and summarize the latest methodologies to achieve these in-depth understandings, such as cryo-electron microscopy technique. We will then follow up with the recent developments to enhance the safety and cycle 
performance of LMBs by designing the electrolytes and the associated functional additives. After that, we will focus on the current progress on the solid-state electrolytes and artificial interface engineering to overcome their shortcomings, such as high interfacial impedance and low ionic conductivity. Then, we also include the latest developments of the advanced host materials for $\mathrm{Li}$ metals with the purpose of resolving the above-mentioned issues. Finally, we will conclude with the remaining challenges and possible research opportunities to facilitate the practical applications of the Li-metal batteries. By doing so, we hope we can draw a road map of the latest developments of the lithium metal anode materials for the readers, and shed light on the possible solutions for the currently existing issues in order to accelerate both fundamental research and practical deployment of the various LMBs.

\section{The Failure Mechanisms of Li Metal Anodes}

Over the past few decades, extensive research has been conducted to gain a deep and fundamental understanding on the failure mechanisms of the Li metal anodes. Generally, the failure of a Li metal anode is recognized to originate from three aspects: a) the high reactivity of Li metal, b) the infinite volume variation during cycling, and c) the dendrite growth caused by the first two factors.

\subsection{High Reactivity of Li Metal}

$\mathrm{Li}$ is a kind of alkali metal element, which has the smallest ion radius among all metal species. This characteristic renders Li with an ultra-high capacity (3860 $\left.\mathrm{mAh} \mathrm{g}^{-1}\right),[6]$ and meanwhile, a very fast transfer kinetics. On the other hand, Li metal has the lowest potential among all the currently known electrode materials,[18] which also makes it highly reactive. Due to these characteristics, $\mathrm{Li}$ metal can easily react with various electrolytes (both the Li-containing salts e.g. $\mathrm{LiAsF}_{6}, \mathrm{LiBF}_{4}$, and 
$\mathrm{LiPF}_{6}$ and the organic solvents e.g. propylene carbonate and ethylene carbonate) to form the so-called solid electrolyte interface (SEI).[20, 21] If this SEI is not chemically stable or mechanically strong enough to prevent the Li metal surface against further reactions, the electrolyte will be continuously consumed and Li metal will be corroded as well, which inevitably leads to the draining of the battery, losing of the active material, and eventually, fast death of the battery.[4, 22 , 23]

\subsection{Infinite Volume Variation during Cycling}

Actually, almost all types of anode materials experience volume changes in case of being charged and discharged (Table 1). Compared to these materials, the direct plating/stripping of host-less Li metal, however, will result in an infinite volume change in an ideal condition that all the Li metal in the anode will be striped and intercalated into the cathode, which will initiate cracks on the SEI.[18, 24] This causes a very high structural instability for the anode, the SEI, and the whole battery configuration.

Table 1. Volume change during cycling of the different anode materials for Li-ion batteries

\begin{tabular}{ccc}
\hline Anode materials & $\begin{array}{c}\text { Volume change rate } \\
\text { during cycling }\end{array}$ & Ref \\
\hline Graphite & $c a .10 \%$ & {$[25]$} \\
Carbon nanotubes & $5 \%$ & {$[26]$} \\
$\mathrm{TiO}_{\mathrm{x}}$ & $2-5 \%$ & {$[27]$} \\
$\mathrm{Li}_{4} \mathrm{Ti}_{5} \mathrm{O}_{12}$ & $<1 \%$ & {$[28]$} \\
$\mathrm{Si}$ & $297-400 \%$ & {$[29,30]$} \\
$\mathrm{Ge}$ & $270-300 \%$ & {$[31]$} \\
$\mathrm{Sn}$ & $257-300 \%$ & {$[32,33]$} \\
$\mathrm{SnO}$ & $259 \%$ & {$[34]$} \\
$\mathrm{Al}$ & $100 \%$ & {$[35]$}
\end{tabular}


For example, in the practical applications, the minimum areal capacity of a single-sided commercial electrode is $3 \mathrm{mAh} \mathrm{cm}^{-2}$, corresponding to a thickness of $c a$. $14.6 \mu \mathrm{m}$ for $\mathrm{Li}$ metal anode.[36] In the case that this amount of $\mathrm{Li}$ is fully stripped (i.e. an infinite volume decrease to 0), a void of at least this thickness will appear between the anode current collector and the separator, which can cause the instability to the electrode structures and significantly alter the actual battery configuration.

\subsection{Dendrite Growth on Li Metal Surface}

Li dendrites are generated by the local polarization during Li plating on the surface of Li metal or current collectors, which impose two major concerns: 1) possible piercing of the separator and leading to internal short circuit of the cell, which makes the battery unsafe (e.g. the "Moli" battery accident), and 2) the formation of the "dead Li", which is caused by the detachment of Li dendrites from the substrate and will lead to the deterioration of battery capacity and efficiency.

Generally, it has been recognized that the formation of Li dendrites is closely related with the uneven surface electrical field during Li plating. Specifically, in this process, a cation concentration gradient is formed in the electrolyte between the two electrodes. When a critical current density $\left(J^{*}\right.$, which is the maximum current density that can be carried by a given electrolyte) is reached, the number of cations in the electrolyte decreases, leading to the disruption of the electrical neutrality around the electrode surface. This causes an unbalanced current distribution on the electrode surface,

resulting in the formation of Li metal dendrites at these sites. The concentration gradient $\left(\frac{\partial C}{\partial x}\right)$ and $J^{*}$ in the battery can be described using the following equations:[37, 38]

$$
\begin{aligned}
& \frac{\partial C}{\partial x}(x)=\frac{J \mu_{a}}{e D\left(\mu_{a}+\mu_{L i^{+}}\right)} \\
& J^{*}=\frac{2 e C_{0} D}{t_{a} L} \text { with } \mathrm{t}_{a}=\frac{\mu_{a}}{\mu_{a}+\mu_{L i^{+}}}
\end{aligned}
$$


where $C_{0}$ is the initial concentration, $L$ is the inter-electrode distance, $t_{a}$ is the anionic transport number, $\mu_{a}$ and $\mu_{L i}{ }^{+}$is the anionic and $\mathrm{Li}^{+}$nobilities, $J$ is the effective electrode current density, $D$ is the diffusion constant, $e$ is the electronic charge.

It has been observed that the growth of Li dendrites is self-enhanced, which means the large dendrites are more prone to become even larger compared with the smaller ones, due to the stronger electric field at the tip of large dendrites with larger curvatures.[35] Thus, Li metal deposits preferentially on the surface with larger curvatures (e.g. on the tip of an existing dendrite or on the cracks of the metal surface), causing the continuous growth of Li dendrites.[36]

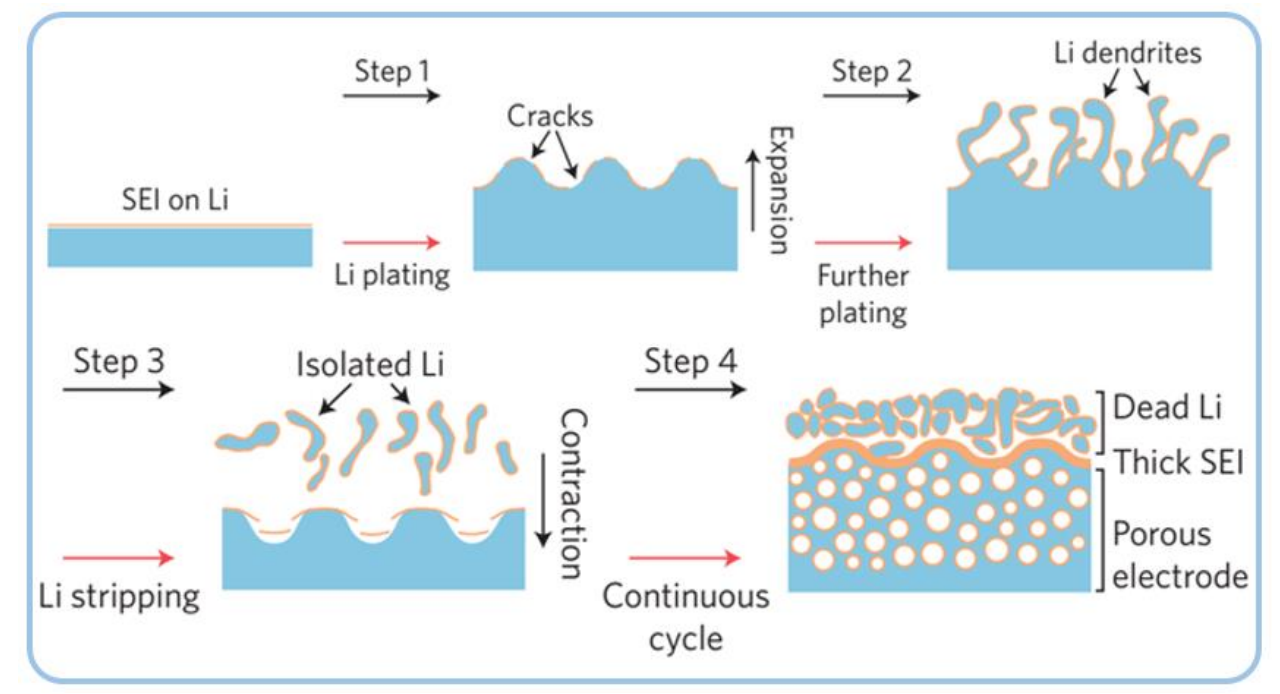

Figure 2. Correlations among the different challenges in the Li metal anode, originate from its high reactivity and infinite relative volume change.[36]

Specifically, during the repeated Li metal striping/plating as described in Figure 2, the volume changes of Li metal initiate cracks on the SEI film.[36] Then, Li starts to preferentially deposit on the cracks, where $\mathrm{Li}$ is unprotected and exposed to the electrolyte. Due to the curvature-induced self-enhancing effect of the Li dendrite growth, the dendrites keep growing at these sites and eventually detach from the substrate Li metal, to form "dead Li". After repeated cyclings, a porous 
Li metal electrode covered by a thick layer of accumulated dead Li will be formed, causing sluggish ion transportation and decreased capacity. To make it even worth, the long Li dendrites may penetrate through the separator and short-circuit the battery, causing severe safety issues, as mentioned above.

\subsection{Advanced Characterizations of Li Metal Anodes}

For a long time, it is a big challenge to study the actual growth process of the Li dendrites or to uncover their detailed formation mechanism during the operation of a LMB, especially at high charge-discharge rates, due to the high sensitivity of the battery components to air. To solve this issue, advanced characterization methods have been recently exploited, such as using a special system, which allowed the transfer of the unpackaged battery components from a glove box into the scanning electron microscopy (SEM) without exposure to ambient environments; [39, 40] directly observing the morphological change of Li metal surface using in-situ atomic force microscopy (AFM); $[41,42]$ and carrying out an in-situ charge-discharge of the electrodes inside the transmission electron microscopy (TEM).[43, 44] Such progresses in characterizing Li dendrites growth have greatly helped us better figure out the failure mechanisms of batteries. 


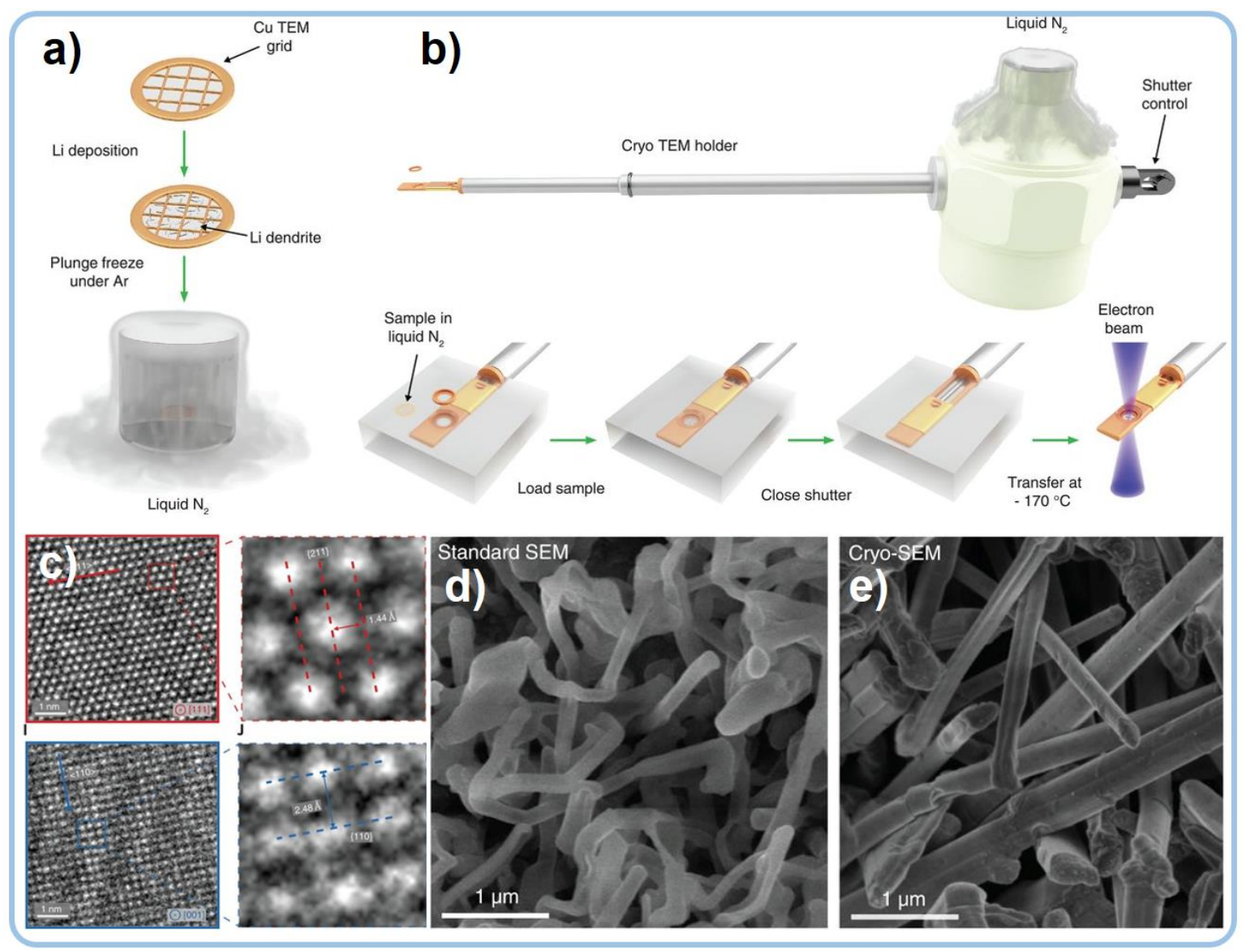

Figure 3. a) Schematic illustration of Li deposition on a copper grid and the freezing process by liquid $\mathrm{N}_{2}$; b) schematic illustration of placing the specimen onto a cryo-TEM holder; c) cryo-TEM images of $\mathrm{Li}$ metal dendrites growing along the $\langle 211\rangle$ (red) and $\langle 110\rangle$ (blue) directions and atomic-resolution TEM images resolving individual Li atoms along the [111] and [001] zone axes; d) standard SEM image of Li metal dendrites after brief exposure to air, with unobservable facets because of the oxidation/corrosion in air; e) cryo-SEM image of Li metal dendrites, showing the dendrite morphology preserved by the cryo-transfer method.[45]

For example, at ambient environments, Li metal can rapidly react with humid air. Besides, due to its low melting point and weak atomic bond, Li atoms are extremely unstable under electron beam irradiation, causing difficulties for characterizations and observations. To overcome these two challenges, researchers have developed a cryo-transfer method and successfully observed the Li 
metal in their native states from different facets at an atomic resolution under cryo-TEM (Figure 3a-c).[45] When conducting the cyro-SEM/TEM observation, Li dendrites are grown on a TEM copper grid directly and then plunged into liquid $\mathrm{N}_{2}$ under $\mathrm{Ar}$ atmosphere to achieve flash-freezing. The specimen is then placed onto the cryo-TEM holder, which is immersed in liquid nitrogen to maintain the delicate structure of $\mathrm{Li}$ dendrite. A closed shutter is employed to prevent $\mathrm{Li}$ from exposing to air and maintain the fine structure of Li dendrite. Only by using cryo-SEM/TEM, rather than the conventional ones, can we found the facets on the dendrites (Figure 3d, e),[45] which show the unit cell type of the dendrite and the crystal orientation of dendrite growth. The development of these novel characterization technologies has led to much more insightful understandings on the formation mechanisms of Li dendrite in LMBs, the actual Li striping/plating process on the Li metals, as well as many new and rational solutions to the above-mentioned issues associated with the Li metal anodes.

To briefly summarize, due to the high reactivity, the Li metal anode can react with the electrolyte to form SEI films on the metal surface, which can, to certain extend, protect the Li metal from being further consumed. On the other hand, the extremely large volume changes of the Li metal anode during cycling result in repeated breaking-apart and re-formation of SEI films, which consequently leads to continuous consumption of Li/electrolyte and uncontrollable $\mathrm{Li}$ dendrite growth during $\mathrm{Li}$ stripping and plating. These comprehensively make the LMBs have a poor performance in cycling stability, coulombic efficiency, and safety, which greatly limits their practical applications. To improve the performance of the Li metal anodes, scientists have conducted a wide range of research, including developing functional electrolyte and additives, synthesizing new solid electrolyte, developing artificial SEI films, and designing novel hosts or substrate materials. 


\section{Organic Liquid Electrolyte Engineering}

The SEI film on the surface of $\mathrm{Li}$, which is formed by the spontaneous reaction between $\mathrm{Li}$ metal and electrolytes upon direct contact or during the charge/discharge processes, determines the electrochemical performance of the Li metal anode to a large extent.[46, 47] Engineering of the electrolytes can directly affect the nature of the SEI and thus modify the Li anode/electrolyte interface, which can significantly influence the performance of the Li metal anode. In the electrolyte, the organic solvents and the Li-contained salts have been identified as the source of the organic species and inorganic species in the SEI film, respectively.[17, 20, 48] Tuning these components in the electrolyte can fundamentally manipulate the actual composition of the SEI. Consequently, in the past few decades, a variety of organic solvents and Li salts have been investigated, leading to a great improvement on the performance of Li metal anodes.

\subsection{Organic solvents design}

Organic solvents, which dissolve Li salts to form a liquid electrolyte, have significant impact on the solubility of the $\mathrm{Li}$ salts and the ionic conductivity of the electrolyte, which can further influence the battery cycling efficiency, the reversible capacity, and safety. Moreover, the organic solvents also determine the main content and the chemical/physical properties of the SEI film,[45] which usually is required to have high $\mathrm{Li}$ ion conductivity and good flexibility to adapt the volume changes during cycling to avoid dendrite growth. The electrolyte solvents studied so far mainly include carbonic ester, carboxylic ester, and alkyl carbonates, with $\mathrm{LiPF}_{6}, \mathrm{LiBF}_{4}, \mathrm{LiClO}_{4}$, $\mathrm{LiN}\left(\mathrm{SO}_{2} \mathrm{CF} 3\right)_{2}$ etc. as the Li salts.[49] The most commonly used solvents and the corresponding battery performances are summarized in Table 2 .

Table 2. Battery performance with electrolytes using different organic solvents. 


\begin{tabular}{|c|c|c|c|}
\hline Solvent & Li salt & $\begin{array}{l}\text { Coulombic } \\
\text { efficiency }\end{array}$ & Ref \\
\hline $\begin{array}{c}\text { Propylene Carbonate } \\
\text { (PC) }\end{array}$ & $\mathrm{LiClO}_{4}$. & 84 & {$[50]$} \\
\hline $\begin{array}{l}\text { Ethylene and dimethyl } \\
\text { carbonates (EC-DMC) }\end{array}$ & $\mathrm{LiPF}_{6}$ & 93 & [51] \\
\hline Tetrahydrofuran (THF) & $\mathrm{LiAsF}_{6}$ & 88 & {$[52]$} \\
\hline $\begin{array}{l}\text { 2-Methyltetrahydrofu } \\
\operatorname{ran}(2-\mathrm{MeTHF})\end{array}$ & $\mathrm{LiAsF}_{6}$ & 96 & [53] \\
\hline $\begin{array}{l}\text { Dimethoxymethane } \\
\text { (DMM) }\end{array}$ & $\mathrm{LiAsF}_{6}$ & 97 & [54] \\
\hline Diethyl ether & $\mathrm{LiAsF}_{6}$ & 98 & [55] \\
\hline
\end{tabular}

Since 1958, PC has been used as the solvent for Li battery electrolytes, due to its high dielectric constant.[21] However, investigation has revealed that the capacity of LMBs with PC-based electrolytes could fade rapidly during cycling,[56] which is because of the reaction between the PC-based solvents and the freshly deposited Li metal,[50] causing the non-uniform morphological change of the Li surface. The uneven growth of the electrodeposited Li will then result in dendrites to further deteriorate the overall battery performance, imposing the low coulombic efficiency and serious safety hazard. Because of this, ether-based electrolytes have been developed to improve the coulombic efficiency and partially alleviate the safety issue, since they can lead to a relatively uniform Li deposition with a smoother surface. The battery performance with various ether-based electrolytes (e.g. THF, 2-MeTHF, diethyl ether, and others) are listed and compared in Table 2. Compared with the PC-based electrolytes, higher coulombic efficiencies can be achieved on these ether-based electrolytes. 
However, the ether solvents still can not completely eliminate the dendrite growth and the capacity retention is usually unsatisfactory after a long cycling.[57] To make it even worse, the ether-based electrolytes tend to decompose at much lower potentials compared to PC (e.g. THF was found to be decomposed at $4.0 \mathrm{~V}$, while PC remained stable at up to $5.0 \mathrm{~V}$ ), which makes them unsuitable for the various newly emerged high-voltage cathode materials. In comparison, ethylene carbonate (EC), a kind of alkyl carbonate, can stably withstand a much wider potential range. By using EC as an additive, the electrolyte solutions can possess a higher ion conductivity and lower polarization. Comprehensively, these merits have made them a hotpot in the research and industry of Li batteries.

Nevertheless, the high melting point of the EC has limited its applications at low temperature. Since 1990s, a diverse range of co-solvents have been introduced into the EC-based electrolytes to expand the application EC-based electrolytes towards lower temperature, including diethoxyethane (DEE),[58] dimethoxyethane (DME),[58, 59] and 2-MeTHF.[60] But none of these solvents was satisfactory, due to the decomposition problem of these ethers as mentioned above.

In recent years, another type of linear carbonates has been developed as the co-solvents in EC-based electrolytes for LMBs. On the one hand, compared with the ether-based co-solvents, these linear carbonates can remain stable in a much wider potential range. On the other hand, compared with the cyclic carbonates, these linear carbonates have combined advantages of low boiling points and low viscosity.[61] Benefited from these, such linear carbonates can be mixed with EC at any ratio, which not only promotes the ionic transporting performance but also significantly extends the operation temperature window of the EC-based electrolytes.[21]

By far, a large number of linear carbonates has been used as the co-solvents, which includes 
dimethyl carbonate (DMC),[61, 62] diethyl carbonate (DEC), $[62,63]$ and ethylmethyl carbonate (EMC), etc.[64-66] Among these choices, ethylene and dimethyl carbonates (EC-DMC) have shown the greatest potential to be used in the LMBs.[17, 51] By adopting it into the electrolyte, the cycling life of a $\mathrm{Li}$ (anode) $\mid \mathrm{LiMn}_{1.9} \mathrm{Co}_{0.1} \mathrm{O}_{4}$ (cathode) battery could achieve 586 cycles, which is much superior compared to 216 cycles of the EC-DME electrolyte, 191 cycles of the EC-DEE electrolyte, and only 110 cycles for the EC-DEC electrolyte.[58] In addition to the various binary solvents, the ternary solvents have been found with a better overall performance, which may be the researching hot spot for the next few years.[67] Consequently, it is clearly shown that choosing suitable functional co-solvents is a very effective strategy to maintain the Li anode's reversibility and thus enhance the overall performance of a LMB.

\subsection{Li Salts Design for Electrolytes}

Besides the organic solvents of the electrolytes, the Li salts also have great impact on the performance of Li metal anode. Li salts can be generally divided into inorganic and organic types. The inorganic $\mathrm{Li}$ salts include $\mathrm{LiClO}_{4}, \mathrm{LiAsO}_{6}$, and $\mathrm{LiPF}_{6}$, while the organic ones include $\mathrm{LiN}\left(\mathrm{CF}_{3} \mathrm{SO}_{2}\right)_{2}$ (LiTFSI), $\mathrm{LiN}\left(\mathrm{SO}_{2} \mathrm{CF}_{3}\right), \mathrm{LiC}\left(\mathrm{SO}_{2} \mathrm{CF}_{3}\right)_{3}$, and others.[49, 68] According to their functions, as summarized in Table $\mathbf{3}$, these Li salts can also be classified as electrolyte solutes, such as $\mathrm{LiPF}_{6}$ [69] and LiTFSI,[70] which mainly provide ionic conductivity for the electrolytes; and electrolyte additives, such as $\mathrm{LiNO}_{3}[71]$ and $\mathrm{Li}_{x} \mathrm{~S}_{y},[72]$ which mainly help generate robust SEI films and further suppress the dendrite growth. In this part, the role of different Li salts will be summarized accordingly. 
Table 3. The specialties of common kinds of Li salts used in electrolyte.

\begin{tabular}{|c|c|c|c|c|}
\hline Function & Li salt & Specialty & $\begin{array}{c}\text { Main } \\
\text { component of } \\
\text { SEI }\end{array}$ & Ref \\
\hline \multirow{5}{*}{ Electrolyte solute } & $\mathrm{LiClO}_{4}$ & $\begin{array}{l}\text { Stable to ambient } \\
\text { moisture but strong } \\
\text { oxidizing }\end{array}$ & $\mathrm{LiCO}_{3}$ & {$[61]$} \\
\hline & $\mathrm{LiAsF}_{6}$ & $\begin{array}{l}\text { Of good cycling } \\
\text { stability but toxic }\end{array}$ & $\mathrm{LiCO}_{3}$ & [73] \\
\hline & $\mathrm{LiBF}_{4}$ & $\begin{array}{l}\text { Less toxic, but of } \\
\text { inferior ion } \\
\text { conductivity }\end{array}$ & $\mathrm{LiF}$ & {$[74]$} \\
\hline & $\mathrm{LiPF}_{6}$ & $\begin{array}{l}\text { Well-balanced } \\
\text { properties but of } \\
\text { thermal instability }\end{array}$ & $\mathrm{LiF}$ & {$[69,75]$} \\
\hline & LiTFSI & $\begin{array}{c}\text { Highly resistant to } \\
\text { oxidation and high } \\
\text { soluble but of moderate } \\
\text { ion conductivity }\end{array}$ & $\begin{array}{c}\mathrm{Li}_{2} \mathrm{~S}_{2} \mathrm{O}_{3} \text { and } \\
\mathrm{Li}_{3} \mathrm{~N}\end{array}$ & [76] \\
\hline \multirow{3}{*}{ Addictive } & $\mathrm{LiNO}_{3}$ & $\begin{array}{c}\text { Forming an even SEI to } \\
\text { protect the anode }\end{array}$ & $\mathrm{LiN}_{\mathrm{x}} \mathrm{O}_{\mathrm{y}}$ & {$[71,77]$} \\
\hline & $\mathrm{Li}_{\mathrm{x}} \mathrm{S}_{\mathrm{y}}$ & $\begin{array}{c}\text { Forming a more robust } \\
\text { and dense SEI }\end{array}$ & $\mathrm{Li}_{2} \mathrm{~S}$ and $\mathrm{Li}_{2} \mathrm{~S}_{2}$ & [71] \\
\hline & $\mathrm{LiNO}_{3}+\mathrm{Li}_{\mathrm{x}} \mathrm{S}_{\mathrm{y}}$ & $\begin{array}{l}\text { Forming a flat and } \\
\text { robust SEI which give } \\
\text { the anode a better } \\
\text { protection }\end{array}$ & $\begin{array}{c}\mathrm{LiN}_{\mathrm{x}} \mathrm{O}_{\mathrm{y}}, \mathrm{Li}_{2} \mathrm{~S} \\
\text { and } \mathrm{Li}_{2} \mathrm{~S}_{2}\end{array}$ & {$[72,78]$} \\
\hline
\end{tabular}




\subsubsection{Li Salts as Electrolyte Solutes}

Basically, Li salts have been used as electrolyte solutes to provide a high ionic conductivity to maintain the uninterruptedly carrying-on of the battery redox reactions.[61, 69, 73-76] The specific characteristics of these $\mathrm{Li}$ salts are summarized in Table 3. For LIBs, $\mathrm{LiClO}_{4}, \mathrm{LiAsF}_{6}, \mathrm{LiBF}_{4}$ and $\mathrm{LiPF}_{6}$ have been used as the electrolyte solutes. Among them, $\mathrm{LiPF}_{6}$ is the most widely used commercial electrolyte solute so far for its balanced properties and high ion mobility.[69] The problem of $\mathrm{LiPF}_{6}$ is the thermal instability. A strict upper temperature barrier $\left(\mathrm{ca} .50^{\circ} \mathrm{C}\right)$ has to be set during battery operation to avoid any possible reactions between $\mathrm{LiPF}_{6}$ and the solvents.[21] Based on $\mathrm{LiPF}_{6}, \mathrm{LiPF}_{6-\mathrm{n}}\left(\mathrm{CF}_{3}\right)_{\mathrm{n}}$ [79] and $\mathrm{LiPF}_{3}\left(\mathrm{C}_{2} \mathrm{~F}_{5}\right)_{3}[80]$ have been synthesized with a better thermal stability, as an alternative for $\mathrm{LiPF}_{6}$. Apart from $\mathrm{LIBs}$, these $\mathrm{Li}$ salts, such as $\mathrm{LiPF}_{6}$ and $\mathrm{LiBF}_{4}$, have also been widely used in the research of Li-S or Li-O $\mathrm{O}_{2}$ batteries. Especially for the Li-S batteries, LiTFSI has been frequently used as the electrolyte solute, which can prevent the formation of the soluble high-order polysulfides and so as to suppress the undesirable shuttling effect.[70]

\subsubsection{Li Salts as Electrolyte Additives}

Li salts used as additives mainly include $\mathrm{LiNO}_{3}, \mathrm{Li}_{\mathrm{x}} \mathrm{S}_{\mathrm{y}}$, and their combination. During the cycling, $\mathrm{LiNO}_{3}$ can be directly reduced to $\mathrm{LiN}_{x} \mathrm{O}_{y}$, which passivates the $\mathrm{Li}$ surface; while $\mathrm{Li}_{\mathrm{x}} \mathrm{S}_{\mathrm{y}}$ can form to $\mathrm{Li}_{2} \mathrm{~S}$ and $\mathrm{Li}_{2} \mathrm{~S}_{2}$, which can generate a dense film to suppress the Li dendrite growth. In the case of simultaneously using these two species, the synergistic effect can be achieved, resulting in a more effective protection for the $\mathrm{Li}$ anodes. To meet the specific requirements of the various battery systems, the careful selection and compositional design of the most suitable additives have to be carried out.

As mentioned, reduction of $\mathrm{LiNO}_{3}$ can form a protective film on the Li metal surface, which can 
passivate the Li surface and suppress the Li dendrite growth.[77, 81] During the reaction between $\mathrm{Li}$ and $\mathrm{LiNO}_{3}$, the formed SEI film is a mixture of the $\mathrm{LiN}_{\mathrm{x}} \mathrm{O}_{\mathrm{y}}, \mathrm{ROLi}$, and $\mathrm{ROCO}_{2} \mathrm{Li}$ (where $\mathrm{R}$ is the organic group such as $-\mathrm{CH}_{3}$ and others) with a uniform and smooth surface, leading to improved stability and cycling performance for the Li metal anodes.[71] Furthermore, in case of a Li-S battery, $\mathrm{LiNO}_{3}$ can also alleviate $\mathrm{Li}$ metal corrosion caused by the shuttle of polysulfides, because of the enhanced inertness of Li metal anode surface. Due to these merits of $\mathrm{LiNO}_{3}$, it has been widely used in various LMBs, resulting in a stable battery cycling performance and high coulombic efficiency.[77, 78]

On the other hand, the $\mathrm{Li}_{\mathrm{x}} \mathrm{S}_{\mathrm{y}}$ additives can also form a dense and thick SEI film on the Li metal surface. Therefore, they have also been considered ideal additives for the electrolytes. Different from the $\mathrm{LiNO}_{3}$-based additives, the $\mathrm{Li}_{\mathrm{x}} \mathrm{S}_{\mathrm{y}}$ reacts with the $\mathrm{Li}$ surface to form an insoluble $\mathrm{Li}_{2} \mathrm{~S}$ and $\mathrm{Li}_{2} \mathrm{~S}_{2}$ layer that is thick and mechanically hard and can thus physically suppress the dendrite growth; while $\mathrm{LiNO}_{3}$ restrains the $\mathrm{Li}$ dendrites by forming a passivation film that is less hard but able to effectively isolate the Li metal surface from contacting the electrolyte.[71, 77]
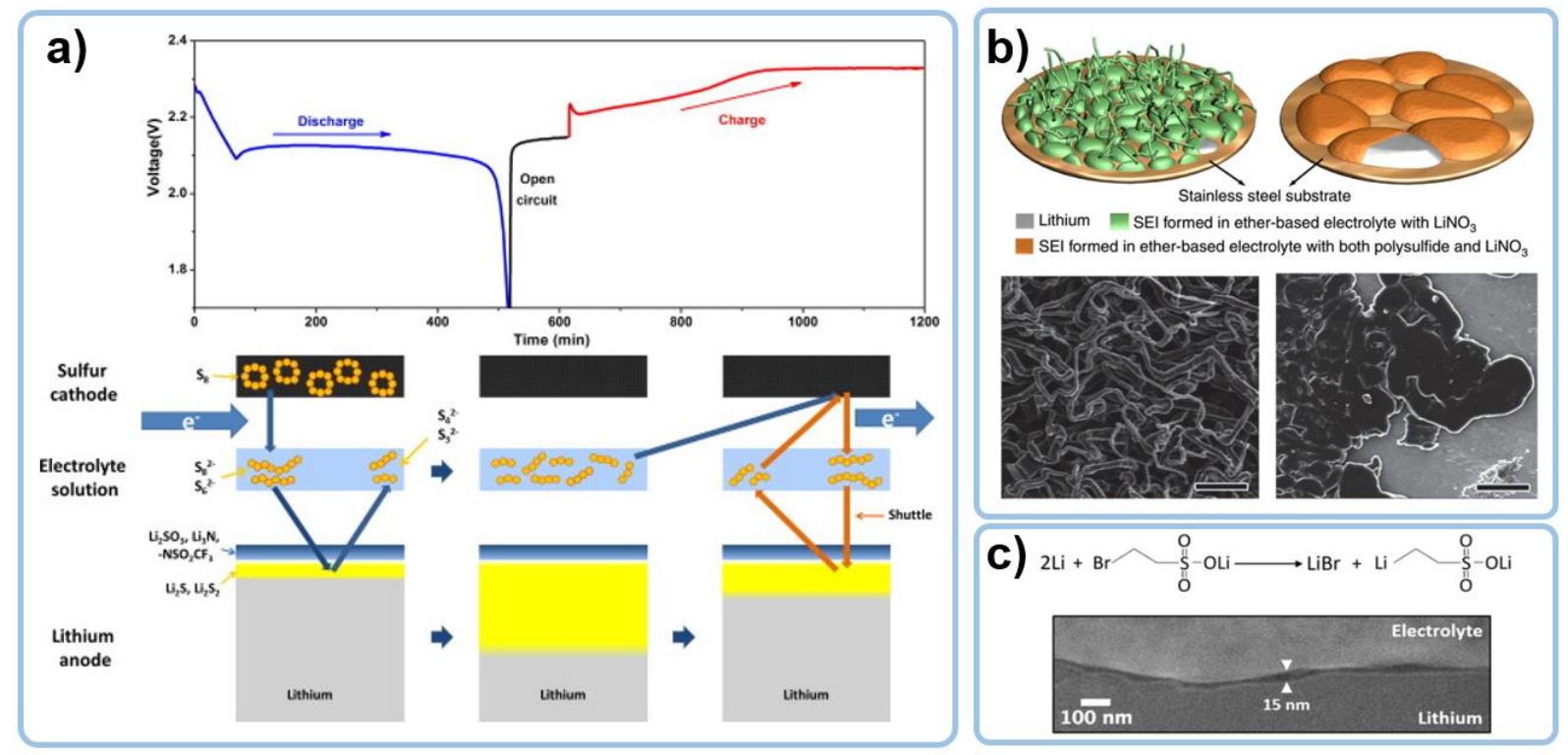

Figure 4. a) Schematic illustration of the surface film behavior on the Li anode in a Li-S battery;[76] 
b) schematic of the morphologies of Li metal surface deposited in different electrolytes without $\mathrm{Li}$ polysulfide and with Li polysulfide and the corresponding SEM images;[78] c) schematic for the reaction of lithium 2-bromoethanesulfonate with $\mathrm{Li}$ metal and the SEM image of the interfacial layer between an intact electrolyte and a Li anode.[82]

Recently, a novel electrolyte system with $\mathrm{Li}_{2} \mathrm{~S}_{6}$ in the LiTFSI-based electrolyte (in a 1,3-dioxolane (DOL) and 1,2-dimethoxyethane (DME, 1:1, v/v)) has been designed for Li-S batteries (Figure 4a).[76] With the addition of the $\mathrm{Li}_{2} \mathrm{~S}_{6}$, a double-layer and bifunctional SEI film was generated. The bottom layer is composed of $\mathrm{Li}$ (di)sulfides that can prevent the contact between LiTFSI and Li metal to avoid their reaction; while the top layer containing $\mathrm{Li}_{2} \mathrm{SO}_{3}$ can further protect the beneath Li (di)sulfides layer from being corroded by the soluble high-order polysulfides in the electrolyte. Therefore, an enhanced Li-S battery cycling stability could be achieved on this electrolyte system.

Moreover, the $\mathrm{LiNO}_{3}$ and $\mathrm{Li}_{\mathrm{x}} \mathrm{S}_{\mathrm{y}}$ additives can be simultaneously employed to obtain an even better synergistic effect to effectively protect the Li anode surface. In the presence of both additives, Li metal can be plated with a flat morphology and without any dendrites in the ether electrolyte, which can not be achieved with $\mathrm{LiNO}_{3}$ alone.[78, 83] The typical Li metal surface morphology obtained in the electrolytes with and without $\mathrm{Li}_{\mathrm{x}} \mathrm{S}_{\mathrm{y}}$ is illustrated in Figure 4b.[78] When merely using $\mathrm{LiNO}_{3}$ as the electrolyte additive, the resulted SEI film did not have sufficient strength to inhibit the growth of $\mathrm{Li}$ dendrites. Upon the addition of $\mathrm{Li}_{\mathrm{x}} \mathrm{S}_{\mathrm{y}}$, a dense SEI film could be formed that was able to prevent the $\mathrm{Li}$ dendrite growth. In this case, a flat $\mathrm{Li}$ deposition with a pancake-like morphology can be achieved, which is smooth and dendrite-free, leading to a high cycling stability of the LMBs. Due to these advantages, such $\mathrm{LiNO}_{3}$ and $\mathrm{Li}_{\mathrm{x}} \mathrm{S}_{\mathrm{y}}$ composite additive has been recently 
used in Li-S batteries.[72, 84] Compared with the sole $\mathrm{LiNO}_{3}$ additive, the SEI formed in the electrolyte with dual additives is not only stronger but also sulfurized, which can provide more pathways for Li ion transport. Under the protection of this sulfurized SEI film, the battery can cycle at a much higher coulombic efficiency of $98 \%$, compared to only $70 \%$ of the one with the routine electrolyte after 200 cycles.[84]

Besides the inorganic Li salts additives, some organic Li salts have also been added into the electrolytes to improve the performance of $\mathrm{Li}$ metal anode. For example, lithium 2-bromoethanesulfonate can react with Li metal surface to form a SEI that is composed of $\mathrm{LiBr}$ and other Li-based organometallic (Figure 4c), which is thin and uniform and can be utilized to stabilize the $\mathrm{Li}-\mathrm{O}_{2}$ batteries from two important aspects.[82] Firstly, it can provide an ideal protection for the Li anode, and secondly, it can also act as an effective redox mediator to lower the overpotential for the oxygen evolution reaction (OER) reaction at the $\mathrm{Li}-\mathrm{O}_{2}$ cathode.

\subsection{Non-Li Additives for Electrolyte Design}

To further modify the surface of Li metal, other functional additives, apart from the various Li salts, have been adopted into the electrolytes. These additives can decompose, polymerize, or adsorb on the surface of metallic Li to tune the properties of the SEI film, $[85,86]$ e.g. the intensity and uniformity, so as to improve the overall performance of LMBs. These additives include: inorganic binary oxides, fluorinated compounds, alkali metal cations, and other additives to be specified.

\subsubsection{Inorganic Binary Oxide Additives}

$\mathrm{CO}_{2}$ is an effective additive for LMBs and has been studied since the 1990 s. Adding $\mathrm{CO}_{2}$ into the electrolyte can generate a hard carbonate-based SEI film, e.g. $\mathrm{LiCO}_{3}$, on the surface of $\mathrm{Li}$ anode, 
which can inhibit the dendrite growth and thus improve the cycling stability of the LMBs.[87, 88] Apart from $\mathrm{CO}_{2}$, water is another widely studied additive to the electrolytes, which can well protect Li metal anode by forming an even and dense SEI film by promoting the hydrolysis of the Li salts or the in-situ formed SEI film.[89] For example, Togasaki and co-workers added a trace amount of water with $\mathrm{CO}_{2}$ into an electrolyte containing $1 \mathrm{M} \mathrm{LiPF}_{6}$ in $\mathrm{EC}$ and DEC.[90] On the one hand, this trace amount of water can promote the hydrolysis of the lithium alkyl carbonate $\left(\mathrm{ROCO}_{2} \mathrm{Li}\right) \mathrm{species}$ in the SEI film to form $\mathrm{LiCO}_{3}$. On the other hand, it can also react with $\mathrm{LiPF}_{6}$ to form $\mathrm{LiF}$ and $\mathrm{HF}$, which will further react with $\mathrm{ROCO}_{2} \mathrm{Li}, \mathrm{Li}_{2} \mathrm{CO}_{3}$, and $\mathrm{Li}_{2} \mathrm{O}$, through the reactions as followed:[91] $2 \mathrm{ROCO}_{2} \mathrm{Li}+\mathrm{H}_{2} \mathrm{O} \rightarrow \mathrm{Li}_{2} \mathrm{CO}_{3}+2 \mathrm{ROH}+\mathrm{CO}_{2}$

$\mathrm{LiPF}_{6}+\mathrm{H}_{2} \mathrm{O} \rightarrow \mathrm{LiF}+2 \mathrm{HF}+\mathrm{PF}_{3} \mathrm{O}$

$\mathrm{ROCO}_{2} \mathrm{Li}+\mathrm{HF} \rightarrow \mathrm{LiF}+\mathrm{ROH}+\mathrm{CO}_{2}$

$\mathrm{Li}_{2} \mathrm{CO}_{3}+2 \mathrm{HF} \rightarrow 2 \mathrm{LiF}+\mathrm{H}_{2} \mathrm{CO}_{3}$

$\mathrm{Li}_{2} \mathrm{O}+$ $2 \mathrm{HF} \rightarrow 2 \mathrm{LiF}+\mathrm{H}_{2} \mathrm{O}$

Compared with the native SEI formed without these additives, the as-formed SEI film containing an increased amount of $\mathrm{LiCO}_{3}$ and $\mathrm{LiF}$ is more uniform and stronger, resulting in an enhanced cycling stability. Specifically, with a suitable content of $\mathrm{H}_{2} \mathrm{O}$ in the electrolyte, the cycling 
performance could possess a maximum increase by $34.5 \%$ as compared to the pristine electrolyte. The coulombic efficiency could reach a maximum of $88.9 \%$ with certain trace amount of water.[91]

\subsubsection{Fluorinated Compound Additives}

Fluorinated compounds are another group of commonly used additives that can improve the performance of the $\mathrm{Li}$ anode by forming a LiF-riched SEI on its surface. LiF is widely acknowledged as an important component for stable and uniform SEI, which can suppress Li dendrite growth.[92, 93] On the one hand, $\mathrm{LiF}$ is electrically insulating and can effectively prevent electrons from passing through the SEI layer. On the other hand, LiF has low diffusion energy and high surface energy for $\mathrm{Li}$ ions so as to facilitate the surface diffusion of $\mathrm{Li}$ ions in the electrodeposition process in an uniform and non-dendritic manner.[92]

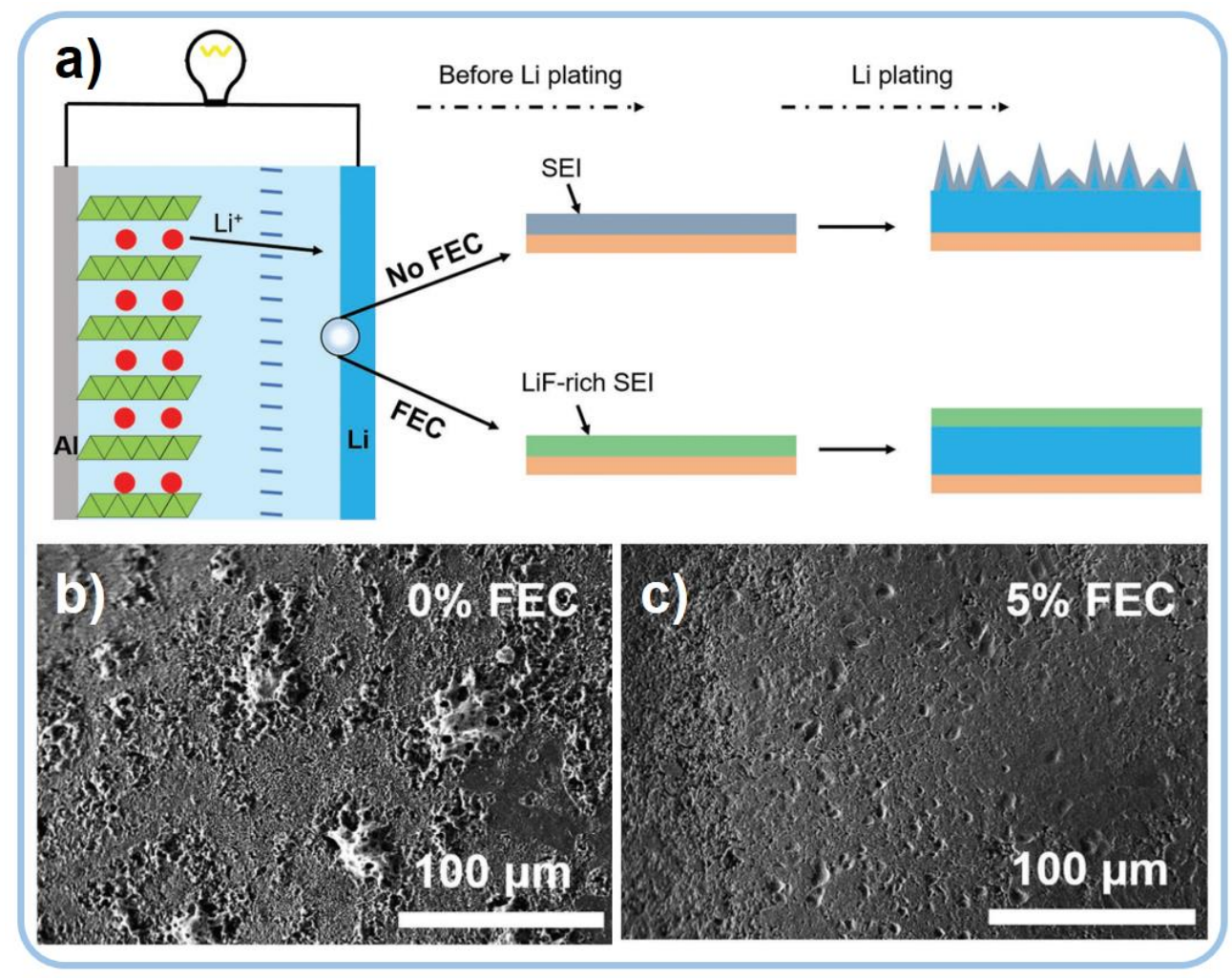

Figure 5. a) Schematic illustration of the effect of FEC additives on a Li metal anode, and the SEM of $\mathrm{Li}$ depositing morphology on $\mathrm{Cu}$ foils after 50 cycles with b) $0 \%$ and c) $5 \%$ FEC content.[94] 
Among the various fluorinated compounds, the fluoroethylene carbonate (FEC) is very attractive and promising as an ideal additive to suppress the Li dendrite formation.[95] Especially. FEC has a very low level of the lowest unoccupied molecular orbital (LUMO) $(-0.87 \mathrm{eV})$, which makes it very easy to be reduced on the surface of Li metal anode to form a LiF-based protective SEI for Li metal. Because of this advantage, it has been recently employed in a variety of LMBs. For example, in a LMB with a high-loading $\mathrm{LiNi}_{0.5} \mathrm{Co}_{0.2} \mathrm{Mn}_{0.3} \mathrm{O}_{2}$ (NMC) cathode, the addition of FEC could result in the formation of a hard and smooth LiF-rich SEI, which was compact and stable enough to keep a uniform and stable Li deposition and at the same time reduce the formation of dead $\mathrm{Li}$, whereas the bare electrolyte could merely yield an uneven surface Li metal surface (Figure 5).[94] Consequently, with this FEC additive, a high coulombic efficiency up to $98 \%$ and a high initial capacity of $154 \mathrm{mAh} \mathrm{g}^{-1}$ (i.e. $1.9 \mathrm{mAh} \mathrm{cm}^{-2}$ ) at $180.0 \mathrm{~mA} \mathrm{~g}^{-1}$ could be achieved.

\subsubsection{Alkali Metal Cations as Additives}

Lately, it has also been found that metal cation additives are capable of suppressing the $\mathrm{Li}$ dendrite growth, which function through a self-healing electrostatic shield mechanism.[96] Specifically, these metal cation additives, such as $\mathrm{Cs}^{+}$and $\mathrm{Rb}^{+}$ions, normally have a lower effective reduction potential than $\mathrm{Li}^{+}$. Therefore, such cations can only be actually adsorbed on the $\mathrm{Li}$ surface rather than being reduced during the battery cycling, forming a positively charged surface on Li metal that can repulse the $\mathrm{Li}^{+}$and generate a so-called electrostatic shield to result in a uniform plating of $\mathrm{Li}^{+}$(Figure 6).[96-98] 


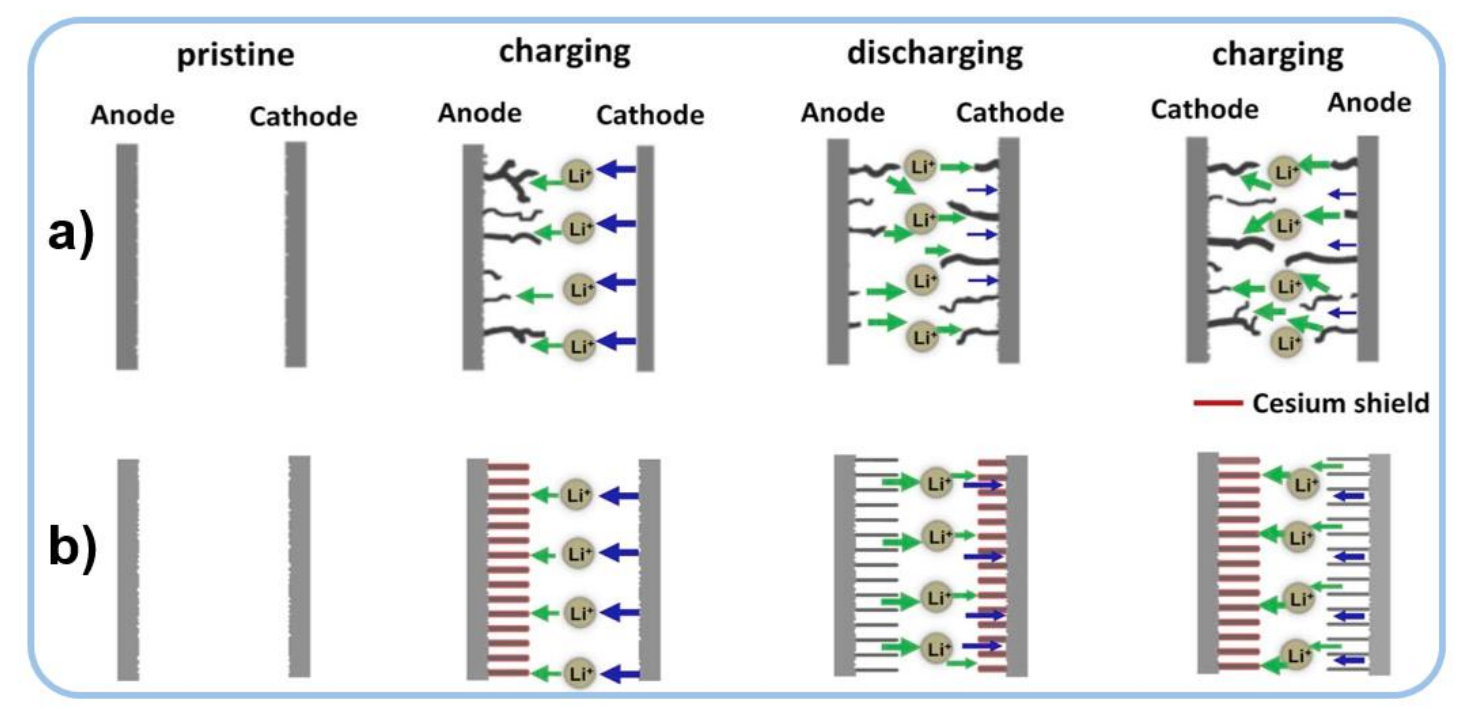

Figure 6. Li deposition models of batteries: a) without and b) with $\mathrm{Cs}^{+}$additives.[98]

Similarly, $\mathrm{K}^{+}$can also be employed for this task.[99, 100] For example, addition of $\mathrm{K}^{+}$into the electrolyte containing lithium oxalyldifluroborate (LiODFB) in EC-DMC can lead to a much more uniform Li deposition and improved cycling stability of the LMB.[99] Differing from the $\mathrm{Cs}^{+}(0.05$ $\mathrm{M})$, only a much lower dosage of $\mathrm{K}^{+}(10 \mathrm{mM})$ could effectively prevent the formation of large $\mathrm{Li}$ dendrites at a high current density of $0.5 \mathrm{~mA} \mathrm{~cm}^{-2}$, which also resulted in a reduced surface density of dendrites by at least 100 folds at a current density of $2.5 \mathrm{~mA} \mathrm{~cm}{ }^{-2}$, further enhancing the assembled LMBs .[100]

\subsubsection{Other Additives}

Apart from the aforementioned chemical methods for $\mathrm{Li}$ surface protection and dendrite prevention by reactively forming protective SEI layers, researchers have also been trying to protect the Li metal surface using inert additives. For example, nano diamonds (ND) have been composited with Li metal through a co-deposition process (Figure 7a).[48] In this study, the negatively charged ND particles can absorb the Li ions on their surface through electrostatic interaction and act as the seeds for Li plating on the substrate surface. The small size of the NDs resulted in homogeneous Li 
deposition, forming a much more homogeneous structure and flatter surface without any dendrites (Figure $\mathbf{7 b}, \mathbf{c})$. Consequently, The LMBs with this ND additives in electrolyte could catch a high coulombic efficiency of $96 \%$.

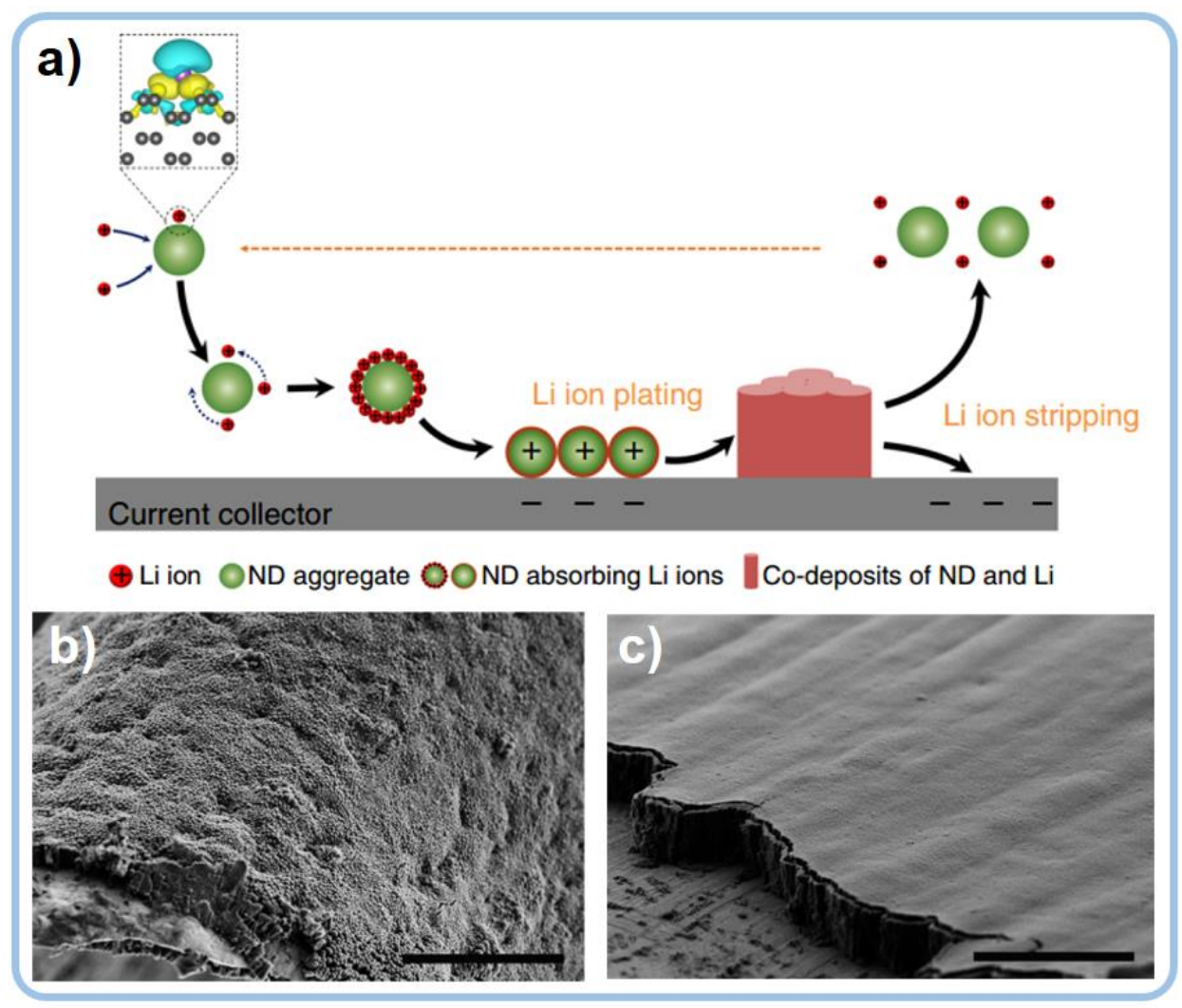

Figure 7. a) Schematic illustration of the co-deposition of Li ions on ND particles, growth of the columnar Li film and the stripping of Li deposits; b, c) SEM images from the cross-sectional views of the electrochemically deposited Li films in the electrolyte without and with ND additives.[48]

Briefly, the design of the liquid electrolytes towards better LMBs can be mainly divided into the above prospects. The EC based organic solvents are still the most widely used ones while the research towards the ether-based co-solvent is still rapidly going on. As for the many different functional additives for the electrolytes, the synergistic effect of $\mathrm{LiNO}_{3}$ and $\mathrm{Li}_{\mathrm{x}} \mathrm{S}_{\mathrm{y}}$ has been acknowledged and much appreciated due to its high effectiveness to protect the Li metal surface, that can greatly benefit different types of LMBs, especially the Li-S batteries. Other additives, such 
as inorganic oxides and fluorinated compounds, are also on quick development. Metal cations that function via a self-healing electrostatic shield mechanism have been proven very effective to form a dendrite-free and reversible $\mathrm{Li}$ metal anode. For a better performance, to consider the solvents, electrolyte solutes, and additives integrally will be essential for developing the next-generation liquid electrolytes for the LMBs.

\section{Solid-State Electrolytes}

Despite the rapid developments of the various liquid electrolytes for Li metal anodes, several major safety issues still remain, such as their high reactivity with Li metal, possible leakage, and high flammability. Even worse, these issues might be further worsened by the flammable nature of $\mathrm{Li}$ metal. Moreover, in these liquid electrolytes, the formation of Li dendrites can hardly be completely avoided. To address these concerns associated with the liquid electrolytes, solid-state electrolytes (SSEs) have been explored with expectations to block the dendrite growth by their high shear modulus and lower reactivity with the Li metal surfaces. Besides these merits, the SSEs are also promising for higher energy densities for Li-metal batteries by getting rid of the unnecessary solvents.[101] For the specific battery systems, the adoption of SSEs could also bring about extra bonus. For example, SSEs can fundamentally overcome the polysulfide shuttle effect in the Li-S batteries, because the SSEs only allow the transfer of Li ions and thus avoid the unwanted loss of both Li metal anode and sulfur cathode. Hence, a rapidly increasing amount of research has been conducted and reported since the SSEs were firstly incorporated into batteries in the 1960s.[102] 


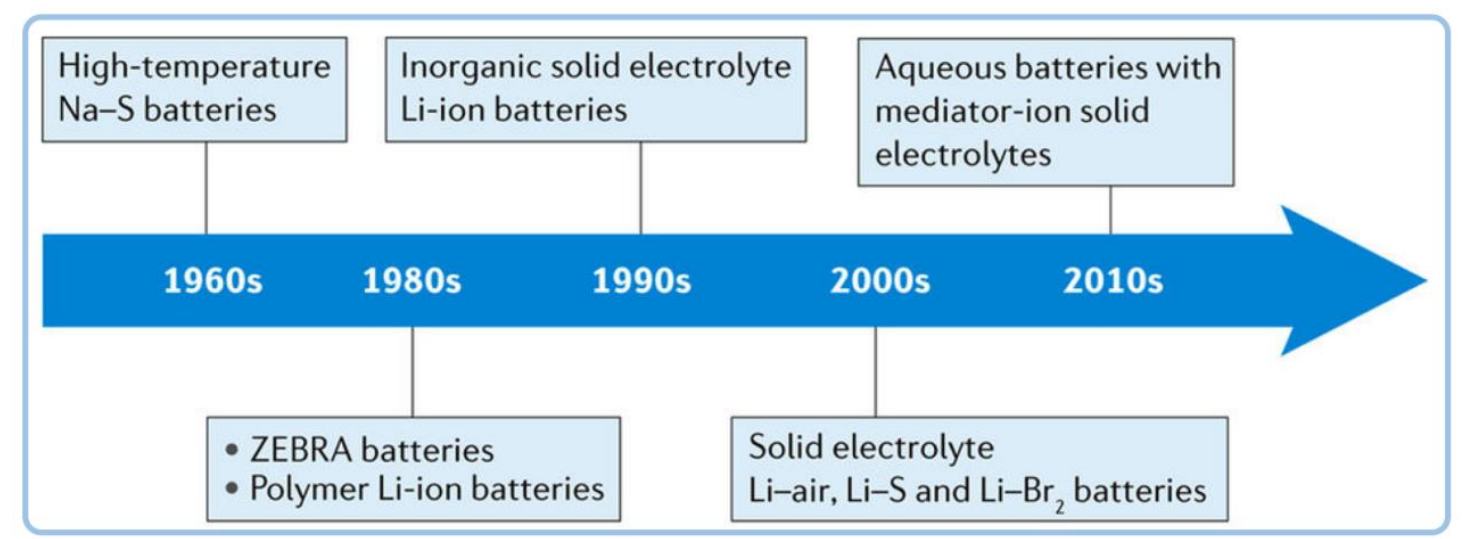

Figure 8. The diagram of historical development of SSEs and the related battery systems.[103]

The track of the developments of SSEs and the related battery systems can be illustrated in Figure 8.[103] Since 1980s, both inorganic and organic SSEs have received increasing attention. Along with the development of both materials and theories, the SSEs have been gradually incorporated into a wide range of electrochemical devices, such as sensors, supercapacitors, and batteries. Since the beginning of this century, researchers have been focusing on the in-depth study of ionic transport mechanism with advanced characterization methods, with the purposes of exploring novel super ionic conductors and extending new applications with these solid ion conducting materials.

However, for the practical application of SSEs, there are still some major obstacles to overcome, including their low intrinsic ionic conductivity at room temperatures and the unsatisfactory compatibility with Li metal anodes. Therefore, the rational design of SSEs should meet several key criteria, such as high ionic conductivity, low interfacial resistance with the electrode active materials, wide electrochemical stable window, good chemical compatibility with both the Li metal and various cathode materials, and low cost.[104, 105] In this section, we will summarize the latest development of the three main types of SSEs: the inorganic, polymeric, and inorganic/polymeric hybrid SSEs. The new methods to improve the ionic conductivity of SSEs will also be introduced. 


\subsection{Inorganic SSEs for LMBs}

Since the lithium phosphorus oxynitride (LiPON) material was for the first time successfully fabricated in 1992,[106] a wide range of Li ion conducting SSEs have been developed, including Na-superionic conductor (NASICON)-type,[107] perovskites-type,[108] sulfide-based,[109] and garnet-type materials [110].

Among the various Li ion-conducting materials, the sulfide-based glass materials frequently possess relatively high ionic conductivity for $\mathrm{Li}$ ions. For example, $\mathrm{Li}_{10} \mathrm{GeP}_{2} \mathrm{~S}_{12}$ is a typical ion conducting sulfide-based glass with outstandingly high Li ionic conductivity of $1.2 \times 10^{-2} \mathrm{~S} \mathrm{~cm}^{-1}$ at room temperature,[111] which was firstly synthesized in 2011 by a stoichiometric reaction of $\mathrm{Li}_{2} \mathrm{~S}$, $\mathrm{GeS}_{2}$, and $\mathrm{P}_{2} \mathrm{~S}_{5}$ at $550{ }^{\circ} \mathrm{C}$ under vacuum. Figure 9 presents and compares the ionic conductivity of the various SSEs and other electrolytes (including both the gel electrolytes and organic liquid electrolytes) as a function of temperatures for practical batteries.[111] It can be noted that even at very low temperatures $\left(\right.$ e.g. $\left.-30{ }^{\circ} \mathrm{C}\right)$, the $\mathrm{Li}_{10} \mathrm{GeP}_{2} \mathrm{~S}_{12}$ material still has a very high conductivity (ca. $1 \mathrm{mS} \mathrm{cm}^{-1}$ ). Nevertheless, in the practical batteries, the $\mathrm{Li}_{10} \mathrm{GeP}_{2} \mathrm{~S}_{12}$ material tends to be reduced by Li metal at low potentials and is commonly sensitive to moisture to generate toxic $\mathrm{H}_{2} \mathrm{~S}$ gas, leading to gassing or other issues for batteries, which needs to be addressed to more effectively utilize such sulfide-type SSE.[112] 


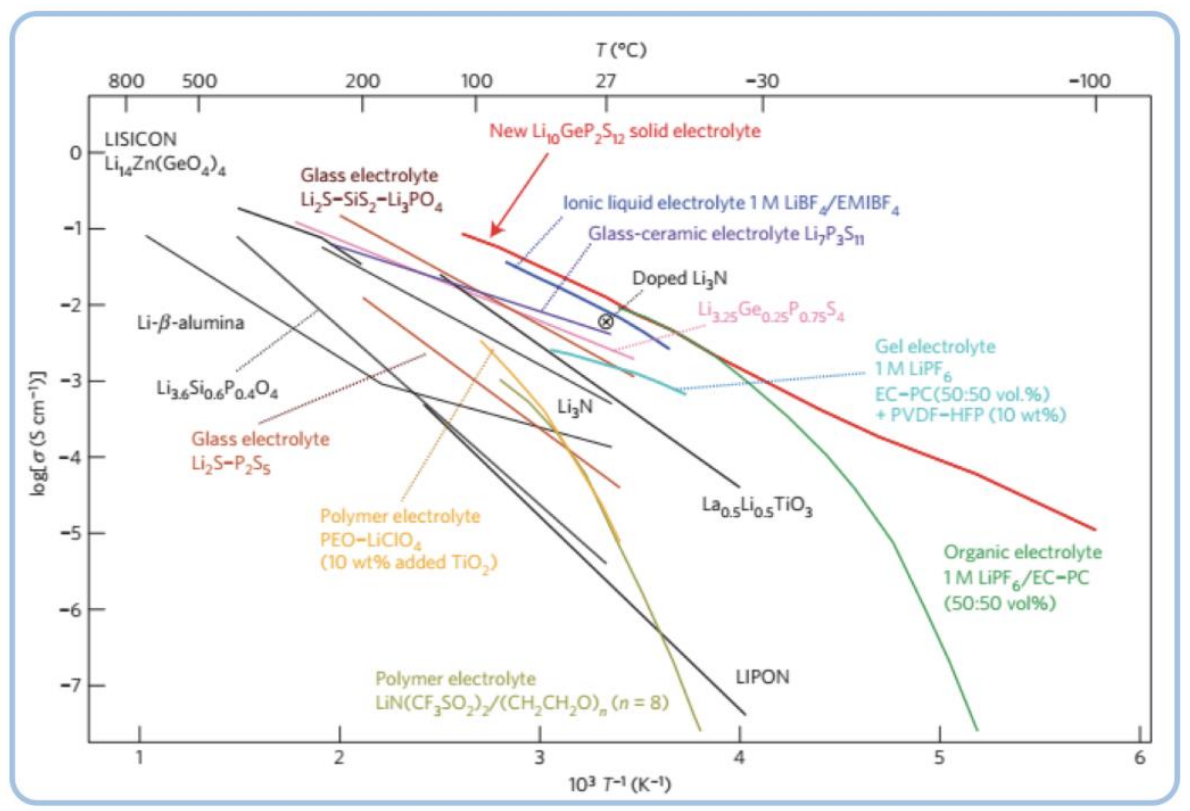

Figure 9. Thermal evolution of ionic conductivity of $\mathrm{Li}_{10} \mathrm{GeP}_{2} \mathrm{~S}_{12}$ phase together with those of other lithium solid electrolytes.[111]

Compared with the sulfide-based SSEs, the oxide solid electrolytes are more stable, free from toxic gas releasing even when exposed to ambient environment.[113] In these materials, the Perovskite $\mathrm{Li}_{3 x} \mathrm{La}_{2 / 3-x} \mathrm{TiO}_{3}$ (LLTO) has a high ionic conductivity up to $10^{-3} \mathrm{~S} \mathrm{~cm}^{-1}$ at room temperature.[114] However, the $\mathrm{Ti}^{4+}$ species in LLTO can be easily converted to $\mathrm{Ti}^{3+}$ or lower valences upon contact with metallic Li anode, which leads to a reactive interface with oxidation of Li metal, causing critical issues for LMBs.

Apart from the sulfide glasses, another frequently studied SSE is the oxide-based and NASICON-type Li ion conductors, which commonly have an $\mathrm{AM}_{2}\left(\mathrm{PO}_{4}\right)_{3}$ formula with the A site usually occupied by $\mathrm{Li}, \mathrm{Na}$, or $\mathrm{K}$ ions and the $\mathrm{M}$ site occupied by Ge, $\mathrm{Zr}$ or Ti ions.[115] In these NASICON-type ionic conductors, the $\mathrm{LiTi}_{2}\left(\mathrm{PO}_{4}\right)_{3}$ system is the representative one and has attracted much interest because of its excellent stability with both atmosphere and water as well as high ionic conductivity $\left(c a .7 \times 10^{-4} \mathrm{~S} \mathrm{~cm}^{-1}\right)$ at room temperature. Moreover, substituting the Ti sites with $\mathrm{Al}$, the $\mathrm{Li}_{1+\mathrm{x}} \mathrm{Al}_{\mathrm{x}} \mathrm{Ti}_{2-\mathrm{x}}\left(\mathrm{PO}_{4}\right)_{3}$ could obtain an even higher ionic conductivity up to $1.3 \times 10^{-3} \mathrm{~S} \mathrm{~cm}^{-1}$ and an 
excellent compatibility with various battery active materials.[116]

Apart from their low ionic conductivity, another typical issue for the SSEs for practical battery applications is their poor interfacial affinity with the electrode active materials. To minimize this interfacial impedance, the sputter-depositing technology has been the most frequently used method to load battery active materials onto the thin SSE films. Kim et al. constructed a $500 \mathrm{~nm}$-thick $\mathrm{LiCoO}_{2}$ (LCO) film by sputter-depositing on the NASICON-type $\mathrm{Li}_{2} \mathrm{O}-\mathrm{Al}_{2} \mathrm{O}_{3}-\mathrm{SiO}_{2}-\mathrm{P}_{2} \mathrm{O}_{5}-\mathrm{TiO}_{2}-\mathrm{GeO}_{2}$ (LICGC) SSEs and systematically studied the microstructural evolution of the LCO cathode and LCO/electrolyte interface in case of heat treatment at different temperatures.[117] It was found that the LCO film was fully crystallized after annealing at $500{ }^{\circ} \mathrm{C}$ and a sharp LCO/LICGC interface without any reaction phases was formed compared with that of $600{ }^{\circ} \mathrm{C}$, which was beneficial for the diffusion of Li ions (Figure 10a-c). The analysis has indicated that although the NACSICON-type electrolyte possessed a relatively lower ionic conductivity compared with the sulfide-based ones, it could still form a stable interface with the electrode active materials, which is essential to improving the cycle performance of LMBs.

Another intensively investigated oxide-based SSE is the garnet-type $\mathrm{Li}_{7} \mathrm{La}_{3} \mathrm{Zr}_{2} \mathrm{O}_{12}$ (LLZO), whose main advantage comes with their wide electrochemical window and excellent stability with Li metal. Similar to other inorganic SSEs, the low ionic conductivity and high interfacial resistance are the two main challenges of the LLZO materials for practical applications. To enhance the ionic conductivity of LLZO, a variety of efforts have been devoted, such as hetero elemental doping [118] or directly fabricating all-solid-state battery assembles on the LLZO solid electrolyte.[119] However, there is still very few satisfying achievements. Recently, minimizing the thickness of SSE has been found to be an effective and promising way that can possibly solve this problem. Yan et al. 
constructed a solid-state battery using a garnet-type LLZO nanoparticle slurry to form a solid electrolyte layer of only several micrometers,[120] two orders of magnitude thinner than those prepared by other methods. The assembled $\mathrm{Li} / \mathrm{LLZO} / \mathrm{LiFePO}_{4}$ cell exhibited an excellent cycle stability at both room temperature and $60{ }^{\circ} \mathrm{C}$ since the thin layer could greatly facilitate Li ion transfer in this battery configuration, thus showing the great potential of this strategy.
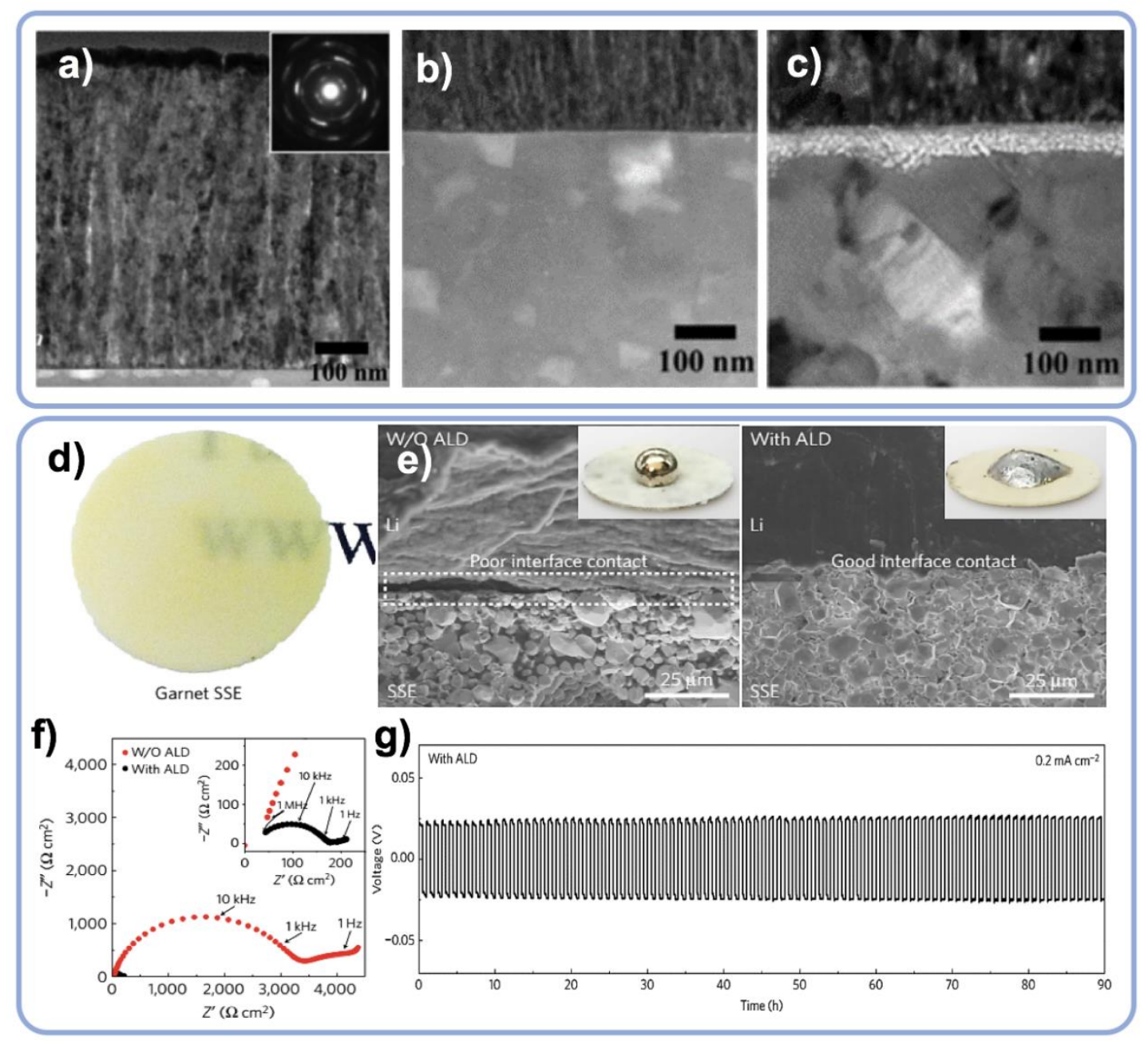

Figure 10. a) Cross-sectional TEM image and the corresponding electron diffraction pattern of LCO film after thermal treatment at $500{ }^{\circ} \mathrm{C}$; TEM images for the LCO/LICGC interface after annealing at b) $500{ }^{\circ} \mathrm{C}$ and c) $600{ }^{\circ} \mathrm{C}$; [117] d) optic image of the LLCZN pellet, e) SEM images of the garnet solid-state electrolyte/Li metal interface with and without ALD, f) EIS result of the symmetric Li/LLCZN/Li cells with and without ALD treatment, and g) galvanostatic cycling results 
of Li/ALD-treated LLCZN/Li cell at a current density of $0.2 \mathrm{~mA} \mathrm{~cm}^{-2}$.[121]

Another aspect that contributes to the high resistance of the LLZO comes from the interface, due to its poor wetting properties with the (molten) Li metal surface, because of the formation of $\mathrm{Li}_{2} \mathrm{CO}_{3}$ on the garnet surface.[122] To avoid it, many strategies have been developed to accelerate the interfacial ionic transfer of LLZO materials. For example, by finely polishing the LLZO surface, the interfacial area specific resistance (ASR) between LLZO and Li metal could be significantly reduced from 960 to $109 \Omega \mathrm{cm}^{2}$, resulting in a much more stable cycling performance than the unpolished LLZO in the same symmetric cell.[122] In another study, Han et al. innovatively applied an ultrathin $\mathrm{Al}_{2} \mathrm{O}_{3}$ coating on the garnet-type $\mathrm{Li}_{7} \mathrm{La}_{2.75} \mathrm{Ca}_{0.25} \mathrm{Zr}_{1.75} \mathrm{Nb}_{0.25} \mathrm{O}_{12}$ (LLCZN) SSE by atomic layer deposition (ALD) method to ameliorate its wettability to the electrode active materials (Figure 10d-e).[121] By doing so, significant enhancement on the interfacial contact between Li metal and the SSE could be achieved to reduce the interfacial ASR to $34 \Omega \mathrm{cm}^{2}$ from $1710 \Omega \mathrm{cm}^{2}$ on the bare garnet LLCNZ SSE. Based on this modified SSE, a symmetric Li/LLCNZ/Li cell has been fabricated with a much decreased impedance (Figure 10f). As a result, excellent cycling performance was achieved for Li plating/stripping at a current density of $0.2 \mathrm{~mA} \mathrm{~cm}^{-2}$ (Figure $\mathbf{1 0 g}$ ). The dramatic reduction in ASR demonstrates that the $\mathrm{ALD}-\mathrm{Al}_{2} \mathrm{O}_{3}$ coating can effectively eliminate the microscopic gaps between Li metal and garnet SSEs, thus providing a very convenient and feasible way to overcome the challenges of garnet-type SSEs.

For these SSEs, although great progress has been achieved so far, the interface problem between inorganic SSEs and Li metal is still significant. Moreover, development of facile and low-cost strategies to fabricate these inorganic SSEs with an outstanding mechanical flexibility and high ionic conductivity at room temperature is very necessary and desirable to make them comparable to the liquid electrolytes counterparts, which indeed requires further exploration. 


\subsection{Solid Polymer Electrolytes}

Compared with the above-mentioned inorganic SSEs, the solid polymer electrolytes (SPEs) have a much better flexibility and can form a more intimate contact with Li metal anodes or the various cathodes. Among all kinds of SPEs, the gel polymer electrolytes (GPEs), especially the poly(ethylene oxide) (PEO) ones, have received the most attention, due to their high solubility of the various Li salts.[123] Unfortunately, such PEO-based GPEs still exhibit a low ionic conductivity, because PEO tends to crystallize at room temperature, suppressing the transport of $\mathrm{Li}$ ions along the polymer chains.[124] This issue can be alleviated by elevating the temperature,[125] modifying the polymer chains to be aligned, or introducing plasticizers.[126, 127] These strategies can improve the conductivity of PEO-based SPEs, however, at the costs of their mechanical strength.

Polymer blending is an effective solution to increase the ionic conductivity of SPEs. This can be attributed to the integrated and comprehensive effects of several factors, including the reduction of crystallinity and the increase of micro ion transfer channels.[128] To achieve this, PEO and polymethyl methacrylate (PMMA) were blended with a poly(vinylidene) fluoride-hexafluoropropylene (PVDF-HFP) co-polymer to form a matrix for GPE (denoted as PE-PM-PVH).[129] Compared with the sole GPEs, this PE-PM-PVH GPE possessed a higher ionic conductivity of $0.81 \mathrm{mS} \mathrm{cm}-1$ at $25^{\circ} \mathrm{C}$ with a superior thermal stability and electrochemical stability. As a result, excellent battery performances (a full cell with Li metal anode and LCO cathode) have been achieved based on this blended GPS, with capacity retention of $98 \%$ after 100 cycles. Besides, Chen et al. reported another composite polymer electrolyte (CPE) membrane by adding an organic cage-type compound cucurbit[6]uril (CB[6]) into PEO based electrolytes via a solvent-free and hot-pressing method. In this $\mathrm{CPE}$, the $\mathrm{CB}[6]$ tends to homogenously disperse in the membrane, 
owing to its natural compatibility with PEO matrix. On the other hand, $\mathrm{CB}[6]$ has carbonyl groups at both ends, which can coordinate with $\mathrm{Li}^{+}$to form complexation and contribute to the uniform deposition of $\mathrm{Li}$ metal onto the electrode.[130] As a result, superior cycling performance was obtained on a LMB using electrolyte for over 200 cycles at $0.5 \mathrm{C}$, which ensured the safe operation of the all-solid-state LMBs.

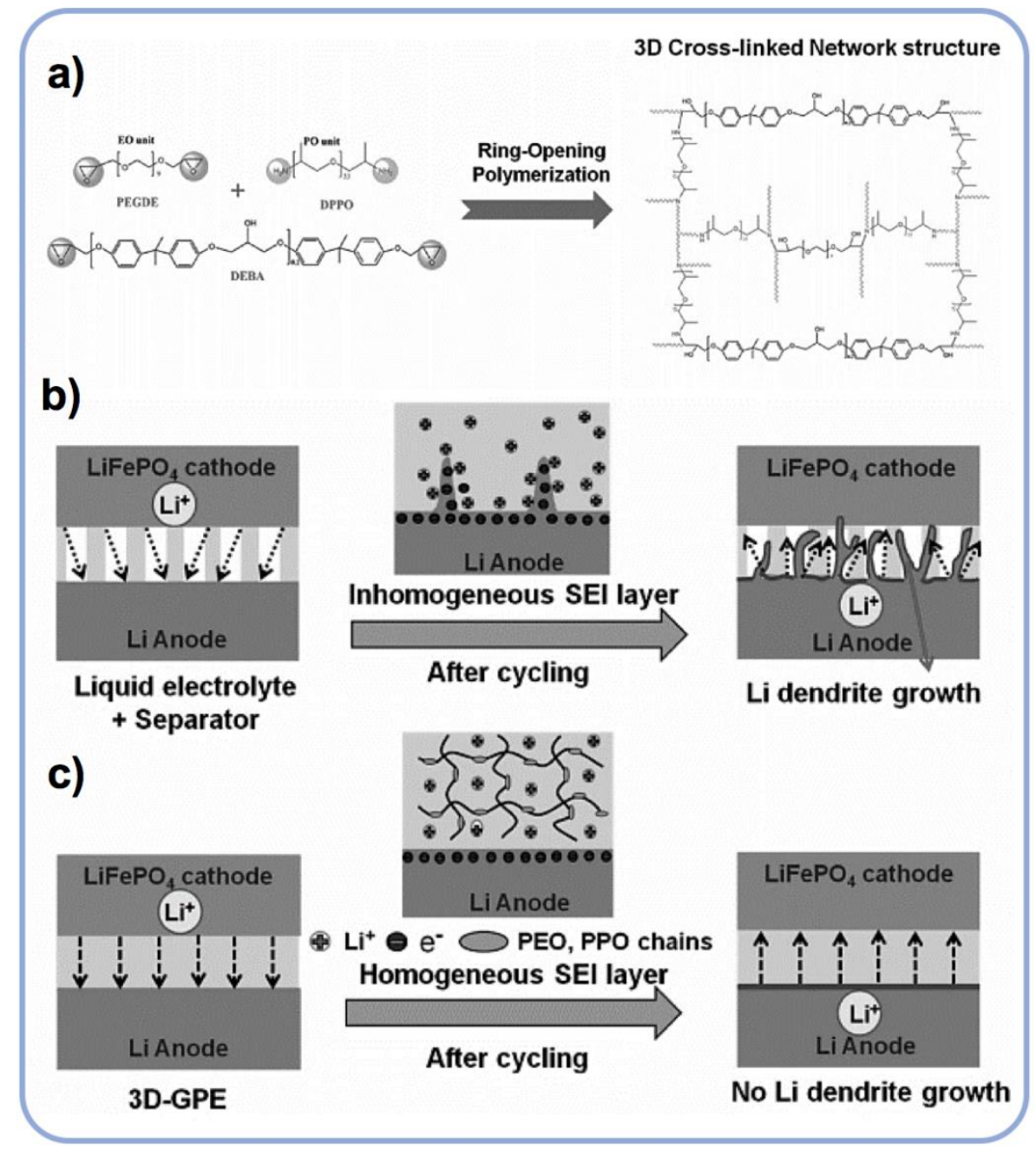

Figure 11. a) Schematic of the synthesis of the 3D-GPE; and the comparison of the Li plating/stripping process on b) liquid electrolyte and c) 3D-GPE.[131]

Apart from further increasing the ionic conductivity, there is also plenty of room to achieve a higher rigidity on these SPEs so as to physically mitigate the Li dendrite growth on the Li metal surface. Generally, incorporating cross-linked structures in the SPEs can lead to a better 
dimensional stability than the chain-like PEO molecules and thus achieve both high ionic conductivity and sufficient stiffness.[132] Based on this, a novel 3-dimensional network GPE (3D-GPE) was recently designed by a ring-opening polymerization reaction of poly(ethylene glycol) diglycidyl ether (PEGDE), diglycidyl ether of bisphenol-A (DEBA), and diamino-poly(propylene oxide) (DPPO) with penetrating linear molecule of poly(vinylidene fluoride-co-hexafluoropropylene) (PVDF-HFP) (Figure 11a).[131] In this process, DBEA served as the supporting framework, in which the PEGDE and DPPO were cross-linked with PVDF-HFP chains embedded in them. Consequently, this ring-opening polymerization method solely generated the cross-linked structure of PEGDE and DPPO, which can ensure a fast ion transport, without any other undesirable by-products. As a result, the obtained 3D-GPE membrane possessed an unprecedented high ionic conductivity of $2.36 \times 10^{-3} \mathrm{~S} \mathrm{~cm}^{-1}$ at $25{ }^{\circ} \mathrm{C}$, even much higher than that of a liquid electrolyte $\left(\mathrm{LiPF}_{6}\right.$ in EC/DMC) soaked in a commercial separator $\left(3.56 \times 10^{-4} \mathrm{~S} \mathrm{~cm}^{-1}\right.$ at $\left.25^{\circ} \mathrm{C}\right)$. Moreover, this compact structure can also promote the formation of a tough and homogeneous SEI layer on Li metal surface, thus effectively restraining the protrusion of Li dendrites as shown in Figure 11b, $\mathbf{c}$.

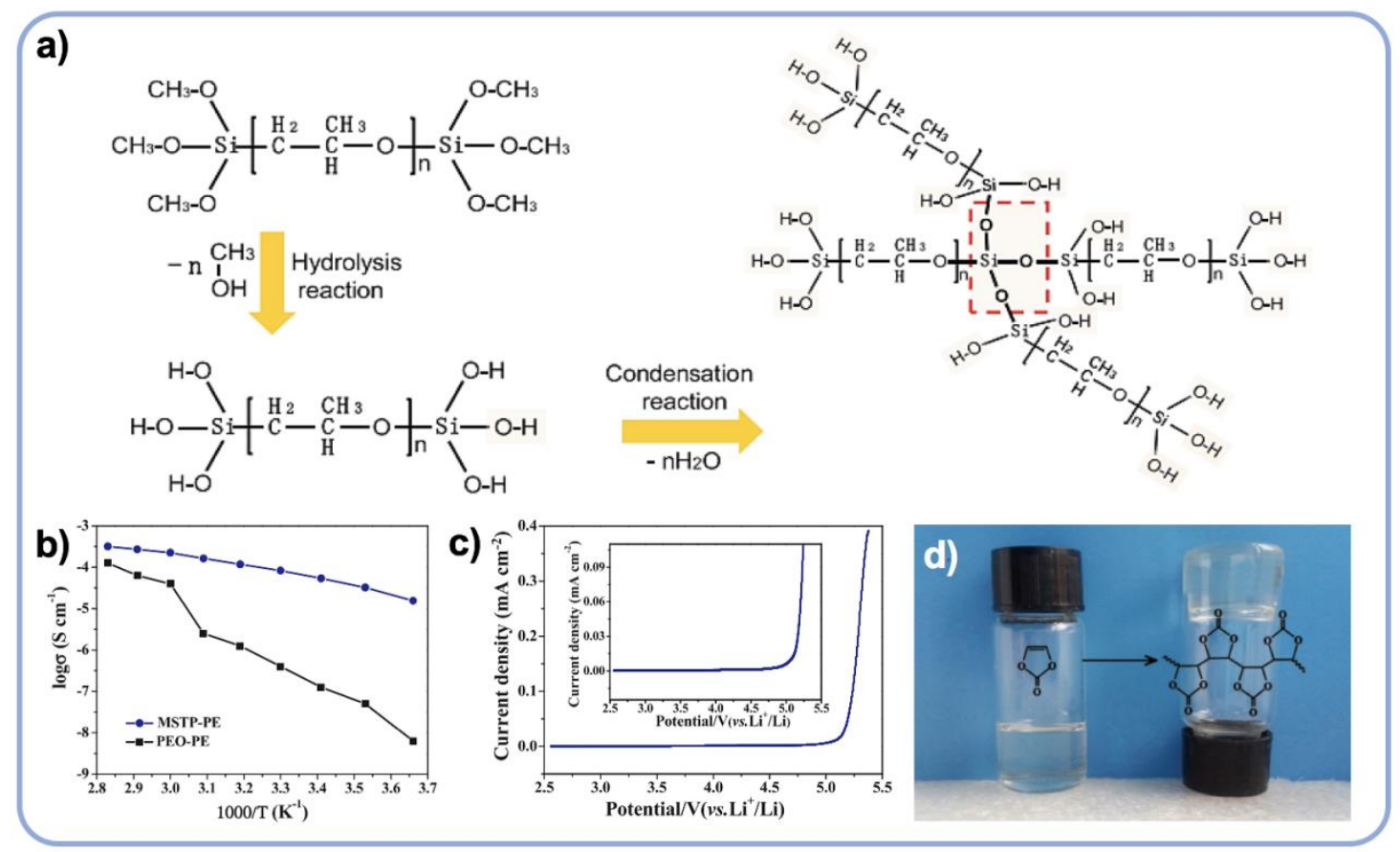


Figure 12. a) The molecular structures of the MSTP monomer and the polymerization process of MSTP-PE; b) ionic conductivities of PEO-PE and MSTP-PE with temperature changes; $\mathbf{c}$ ) linear sweep voltammetry profile of the MSTP-PE at a scan rate of $1 \mathrm{mV} \mathrm{s}^{-1}$. Insert is the local enlarged view for the current density within stable electrochemical window;[133] and d) Image of in-situ polymerization of liquid vinylene carbonate (VC) into PVCA after heating at $60{ }^{\circ} \mathrm{C}$ for 24 h.[134]

Compared with the PEO-based SPEs, the polysiloxane (PSI)-based ones have higher ionic conductivity, lower glass transition temperature $\left(\mathrm{T}_{\mathrm{g}}\right)$, as well as better flexibility, because of the long polysiloxane backbones that can supply adequate diffusion pathways for $\mathrm{Li}$ ions, and thus have been widely studied as the solid polymer electrolytes for LMBs.[19] A main problem of the conventional PSI-electrolyte is the difficulty in battery assembling due to its liquid/gelatinous state at room temperature. To deal with this issue, a novel modified silyl-terminated polyether (MSTP-PE) has been fabricated by using TEGDME as the plasticizing agent and LiTFSI as the Li salt (Figure 12a).[133] This novel MSTP-PE material exhibited a high ionic conductivity of $3.6 \times 10^{-4} \mathrm{~S} \mathrm{~cm}^{-1}$ at $25{ }^{\circ} \mathrm{C}$ (Figure 12b), stable electrochemical window of up to $5.0 \mathrm{~V}$ (Figure 12c), and superior thermal stability. The excellent characteristics of this MSTP-PE electrolyte can also be reflected from the very good battery performance. When it is assembled into a $\mathrm{LiFePO}_{4} / \mathrm{MSTP}-\mathrm{PE} / \mathrm{Li} \mathrm{LMB}$, a highly reversible specific capacity of $170 \mathrm{~mA} \mathrm{~h} \mathrm{~g}^{-1}$ at $1 \mathrm{C}$ and excellent thermal stability (126 and $154 \mathrm{mAh} \mathrm{g}^{-1}$ at $5{ }^{\circ} \mathrm{C}$ and $60{ }^{\circ} \mathrm{C}$, respectively) can be simultaneously achieved, demonstrating the application potential of this MSTP-PE electrolyte for high performance LMBs.

Moreover, some other rigid molecular species have also been introduced as the crosslinkers into the PSI-based SPEs for a better mechanical stability. For example, gallic acid molecules have been cross-linked with an ion conducting PSI derivative through a thiolene click reaction.[135] 
Because of the rigid aromatic rings in the gallic acid molecule, the resulted SPEs exhibited an enhanced mechanical stability as well as greater thermal stability up to $150{ }^{\circ} \mathrm{C}$ compared with the pristine PSI electrolytes that exploded after $15 \mathrm{~min}$ at $150{ }^{\circ} \mathrm{C}$ in a coin cell, which indicated that the Li dendrite growth was more effectively mitigated in this rigid and mechanically strong SPEs reinforced by the gallic acid molecules..

Another type of PSE is the polycarbonate (PC)-based ones, which have relatively higher ionic conductivity ( $c a$. the order of $10^{-5} \mathrm{~S} \mathrm{~cm}^{-1}$ ), moderate electrochemical stability window, and better compatibility towards the Li anode compared with the conventional PEO-based SPEs. Commonly, such PC-based PSEs are fabricated by a solution casting technique, which involves toxic solutions and is time-consuming. Aimed at this issue, novel facile in-situ polymerization methods have been developed. In this method, the liquid precursors are injected into the batteries followed by in-situ polymerization to directly fabricate the PC-based PSEs inside a battery.[136] For example, a poly(vinyl carbonate) (PVCA)-based SPE was fabricated through an in-situ radical polymerization process (Figure 12d).[134] This PVCA-SPE possessed a decent electrochemical stability window of $4.5 \mathrm{~V}$ and ionic conductivity of $9.82 \times 10^{-5} \mathrm{~S} \mathrm{~cm}^{-1}$ at $50{ }^{\circ} \mathrm{C}$. When assembled into LCO/PVCA-SPE/Li battery, a high specific capacity of $146 \mathrm{mAh} \mathrm{g}^{-1}$ at $0.1 \mathrm{C}$ at $50{ }^{\circ} \mathrm{C}$ was achieved, which maintained $99.3 \%$ after 150 cycles. Using similar radical-assisted and in-situ polymerization technique, a poly(diethylene glycol carbonate) dimethacrylat (PDEC-DMA) macromonomer was also synthesized, which was subsequently converted into an interpenetrating network polymer electrolyte (IPN-PDEC).[137] The obtained polymer electrolyte possessed a remarkable mechanical property (Young's modulus of $2.0 \mathrm{GPa}$ ), a high ionic conductivity of $1.64 \times 10^{-4} \mathrm{~S} \mathrm{~cm}^{-1}$, and a good electrochemical stability up to $4.5 \mathrm{~V}$. A $\mathrm{LiFePO}_{4} / \mathrm{IPN}-\mathrm{PDEC} / \mathrm{Li}$ coin cell could be stably cycled at 
$0.2 \mathrm{C}$ with a high specific capacity of $142 \mathrm{mAh} \mathrm{g}^{-1}$ and coulombic efficiency of $99 \%$ after 100 cycles. These successes thus demonstrate that the polycarbonate-based electrolytes have great potential, especially for the high-temperature LMBs.

These numerous endeavors discussed above have significantly increased the ionic conductivity through polymer blending or in-situ polymerization. The mechanical strength of SPEs can also be enhanced by introducing some rigid molecular species (e.g. gallic acid), which in turn improved the cycling and safety properties of the battery. However, their relatively low stability with Li metal and the inferior rigidity compared with the inorganic ones still require future studies and improvements for practical application in LMBs.

\subsection{Inorganic/Polymer Hybrid Electrolytes}

As discussed above, the specific types of electrolytes have the respective shortcomings, such as the low ionic conductivity and inferior mechanical modulus of polymer electrolytes and the poor adhesion of inorganic electrolytes towards Li anode. To remedy their individual defects, integrating the inorganic and polymer SSEs by introducing the strong ceramic fillers into the soft polymer matrix via different protocols seems a very promising method, with the advantages in multiple aspects. 

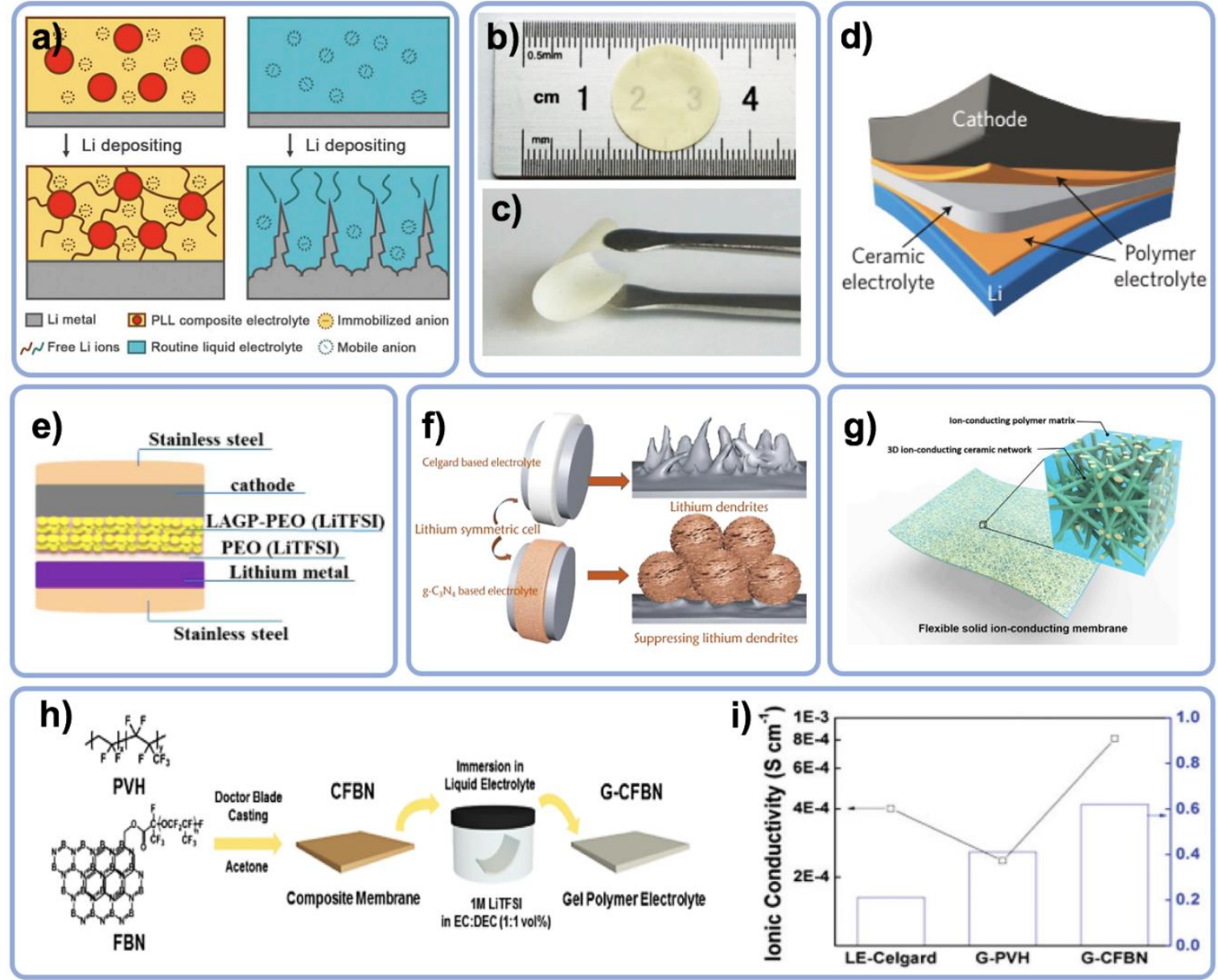

Figure 13. a) Schematic of Li depositing on Li metal anode with PLL electrode and routine liquid electrolyte, digital images of the b) flat and c) bended PLL electrolyte;[138] d) schematic diagram of PCPSE architecture in an all-solid-state battery;[139] e) illustration of an all-solid-state battery with LAGP-PEO (LiTFSI) hybrid electrolyte;[140] f) Li dendrite growth and suppression on symmetric cells with or without $\mathrm{g}-\mathrm{C}_{3} \mathrm{~N}_{4} ;[141] \mathrm{g}$ ) schematic of the $3 \mathrm{D}$ garnet-polymer composite architecture;[142] h) protocol for fabrication of gelatinous composite PFPE-functionalized BNNFs (G-CFBNs); i) ionic conductivity and $\mathrm{Li}^{+}$transference number of LE-Celgard, G-PVH, and G-CFBN at $25^{\circ} \mathrm{C} .[143]$

Firstly, introducing inorganic species into the polymer SSEs can effectively improve their mechanical strength while maintaining certain degree of flexibility. For example, a PEO-lithium bis(trifluoromethylsulphonyl)imide (LiTFSI)-LLZTO (PLL) composite electrolyte was fabricated 
by mixing the garnet-type electrolyte $\left(\mathrm{Li}_{7-\mathrm{x}} \mathrm{La}_{3} \mathrm{Zr}_{2-\mathrm{x}} \mathrm{Ta}_{\mathrm{x}} \mathrm{O}_{12}\right)$ particles with $\mathrm{Li}$ salt and cross-linked PEO substrate.[138] This PLL electrolyte was anion-immobilized, which contributed to the uniform $\mathrm{Li}$ ions distribution and effective dendrite inhibition (Figure 13a). On the one hand, the polymer-Li salt matrix in it ameliorated the interfacial wettability between the electrolyte and Li metal surface. On the other hand, the PLL's inherited high rigidity from the ceramic fillers could provide a strong suppression against dendrites growth.[144] Notably, this composite electrolyte was well freestanding but mechanically flexible (Figure 13b, c), advantageous to application on the flexible all-solid-state batteries. And these merits of the composite membrane were further confirmed by its excellent performance in an all-solid-state $\mathrm{LiFePO}_{4} / \mathrm{PLL} / \mathrm{Li}$ battery, which achieved a specific capacity of $155 \mathrm{mAh} \mathrm{g}^{-1}$ and coulombic efficiency of $99 \%\left(0.1 \mathrm{C}\right.$ at $\left.60{ }^{\circ} \mathrm{C}\right)$ with $87 \%$ capacity retention after 100 cycles.

Alternatively, apart from directly blending the polymer with the ceramic particles, a layer-structured polymer/ceramic/polymer sandwich electrolyte (PCPSE) composed of cross-linked poly(ethylene glycol) methyl ether acrylate (CPMEA) and $\mathrm{Li}_{1.3} \mathrm{Al}_{0.3} \mathrm{Ti}_{1.7}\left(\mathrm{PO}_{4}\right)_{3}$ (LATP) has been developed (Figure 13d).[139] In this architecture, the ceramic layer can decrease the interfacial double-layer electric field between the surface of Li metal and the polymer; while the soft polymer layer can promote the uniform diffusion of $\mathrm{Li}$ ions at the interface by providing an intimate contact between the electrolyte and the electrode surface. $\mathrm{LiFePO}_{4} / \mathrm{Li}$ all-solid-state cells were assembled with both CPMEA and PCPSE membranes and the obtained initial discharge capacities were close (around $130 \mathrm{mAh} \mathrm{g}^{-1}$ at $0.2 \mathrm{C}$ and $120 \mathrm{mAh} \mathrm{g}^{-1}$ at $0.5 \mathrm{C}$, respectively). However, the capacity of the $\mathrm{LiFePO}_{4} / \mathrm{CPMEA} / \mathrm{Li}$ cell faded rapidly to $70 \mathrm{mAh} \mathrm{g}^{-1}$ after 325 cycles, while the one using the PCPSE electrolyte still retained $102 \mathrm{mAh} \mathrm{g}^{-1}$ after 640 cycles with a high coulombic efficiency of nearly $100 \%$, verifying the better electrochemical stability of PCPSE. Similarly, a 
$\mathrm{Li}_{1.5} \mathrm{Al}_{0.5} \mathrm{Ge}_{1.5}\left(\mathrm{PO}_{4}\right)_{3}$-poly(ethylene oxide) (LAGP-PEO) composite solid electrolyte with a buffer layer of PEO (LiTFSI) has also been proposed, which also effectively balanced the modulus and surface adhesion (Figure 13e),[140] indicating that the PCPSE is feasible for high-performance LMBs.

Nanoscale inorganic fillers have relatively larger contact areas with polymer electrolytes and can effectively inhibit the crystallization of polymer phases, which can increase the transport routes for $\mathrm{Li}$ ions and thus are possibly beneficial to induce an extra ionic conductivity compared to the sole polymers.[145] In some cases, even some passive ceramic nanoparticles, such as $\mathrm{TiO}_{2}[146]$ and graphitic carbon nitride $\left(\mathrm{g}-\mathrm{C}_{3} \mathrm{~N}_{4}\right)$, [141] have been proven effective for this purpose. Moreover, these nanostructured inorganic electrolytes can also effectively suppress Li dendrite formation due to their high mechanical strength (Figure 13f).[141] However, these passive nanoparticles themselves are non-conductive for Li ions, which means the ionic migration pathways are only confined within the interphase regions between the particles and polymers, making the actual ionic conductivity lower than the ones using the ionic-conducting nanoparticles, such as $\mathrm{Li}_{6.4} \mathrm{La}_{3} \mathrm{Zr}_{1.4} \mathrm{Ta}_{0.6} \mathrm{O}_{12}$ (LLZTO),[147] $\mathrm{Li}_{1.3} \mathrm{Al}_{0.3} \mathrm{Ti}_{1.7}(\mathrm{PO} 4)_{3}$ (LATP),[148] and organic polymer nanoparticles [149]. Therefore, for example, the ion conductive $\mathrm{Li}_{1.3} \mathrm{Al}_{0.3} \mathrm{Ti}_{1.7}\left(\mathrm{PO}_{4}\right)_{3}$ (LATP) nanoparticles have been dispersed in a PEO matrix to enhance the ionic conductivity to $1.7 \times 10^{-4} \mathrm{~S} \mathrm{~cm}^{-1}$ at room temperature, which was 1 to 2 orders of magnitude higher than that of the PEO matrix.[150]

Other low-dimensional (1D nanofibers or 2D nanoflakes/sheets) inorganic SSE nanomaterials have also been developed, due to their larger surface area and better mechanical integrity in comparison with the $\mathrm{OD}$ ones, which contributes to a more efficient ionic conduction network in SPEs and is able to further enhance the ionic conductivity.[151] For example, the garnet-type 
$\mathrm{Li}_{6.4} \mathrm{La}_{3} \mathrm{Zr}_{2} \mathrm{Al}_{0.2} \mathrm{O}_{12}$ (LLZO) nanofibers were incorporated into a polymer electrolyte of PEO/Li salt to form a garnet-polymer composite electrolyte with a 3D network structure (Figure 13g).[142] This rationally designed hybrid structure provided both continuous Li ion transfer channels from the LLZO nanofibers and mechanical flexibility from the polymer matrix, resulting in a high ionic conductivity of $2.5 \times 10^{-4} \mathrm{~S} \mathrm{~cm}^{-1}$ at room temperature and superior structural strength to impede $\mathrm{Li}$ dendrites growth. Recently, Shim et al. reported another composite GPE, which was composed of perfluoropolyether (PFPE) functionalized boron nitride nanoflakes (BNNFs) in the P(VdF-co-HFP) (PVH) matrix (Figure 13h).[143] A very small amount of such BNNFs (0.5 wt.\%) in the composite GPE could induce a significantly enhanced performance, including a high ionic conductivity of $4 \times 10^{-4} \mathrm{~S} \mathrm{~cm}^{-1}$ at $25{ }^{\circ} \mathrm{C}$ (twice of the conventional liquid electrolyte with a commercialized separator as shown in Figure 13i), super high mechanical performance (Young's modulus of $110 \mathrm{GPa}$ and tensile strength of $53 \mathrm{MPa}$ ), and promising dendrite-inhibiting capability. Due to these advantages, the LMBs with this composite GPE showed both high capacity and good cycling stability that were even much superior to the one using conventional liquid electrolytes. This meritorious work thus rendered a highly desirable strategy that was very promising for commercial applications of LMBs in the future.

\section{Interfacial Engineering of Li Anode and Electrolyte/Separator}

As aforementioned, the SEI films can spontaneously form on the surface of Li metal anode through reactions between the Li metal surface and the electrolyte (i.e. the Li salt and/or the organic solvents), which to certain degree provides some protection to the Li metal surface and suppresses the dendrite growth. However, such spontaneously formed "native" SEI films are normally too thin and fragile for the long-term cycling. Consequently, to intentionally design artificial SEI films with 
better chemical stability and physical robustness is a more ideal solution to inhibit the problematic Li dendrite and unnecessary consumption of electrolytes.

According to the formation mechanisms, the artificial SEI films can be divided into the in-situ artificial SEI films that are directly formed on the Li metal surface, and the ex-situ artificial SEI films, which are individually fabricated and then transferred/attached onto the Li metal surface or generated via the pre-treatment of the Li metal anode before the initial operation of the battery. In addition to designing artificial SEI films on Li metal anode, modification of the separator interface can achieve similar effect and thus suppress the dendrite growth and improve the performance of the LMBs.

\subsection{In-situ Formed Artificial SEI Films}

The in-situ artificial SEI films are usually generated via reactions between the Li metal surface and the intentionally added additives in the electrolytes (as mentioned in 3.2.2, 3.3.1 and 3.3.2), such as Li salts, inorganic oxides, or fluorinated compounds. Compared with the native SEI films, these in-situ formed artificial SEI films are thicker and stronger, with a higher mechanical strength. A series of in-situ artificial SEI films with various compositions have been studied, including $\mathrm{Li}_{2} \mathrm{~S}_{\mathrm{x}}$-riched SEI film and LiF-riched SEI film, $[84,94]$ which have effectively improved the performance of the Li metal anode in different battery systems.

Among the many reported in-situ artificial SEI films, those organic-inorganic hybrid ones can more effectively improve the performance of Li metal anode. Particularly, the organic component can provide sufficient elasticity to cope with the volume changes during the cycling. For example, through the in-situ reaction between the Li metal foil and a mixture of 1-chlorodecane vapor and oxygen/carbon dioxide gas, a mechanically strong hybrid artificial SEI film containing $\mathrm{RCO}_{3} \mathrm{Li}$ and 
$\mathrm{LiCl}$ was generated on the surface of the Li metal anode.[152] This artificial SEI is thick (over 30 $\mu \mathrm{m})$ but conductive to Li ions, which can thus effectively suppress Li dendrite growth. The cycling life of the $\mathrm{Li}-\mathrm{O}_{2}$ batteries with this hybrid SEI film can be significantly enhanced to over 340 cycles, almost 7 folds higher compared with bare Li metal anode.

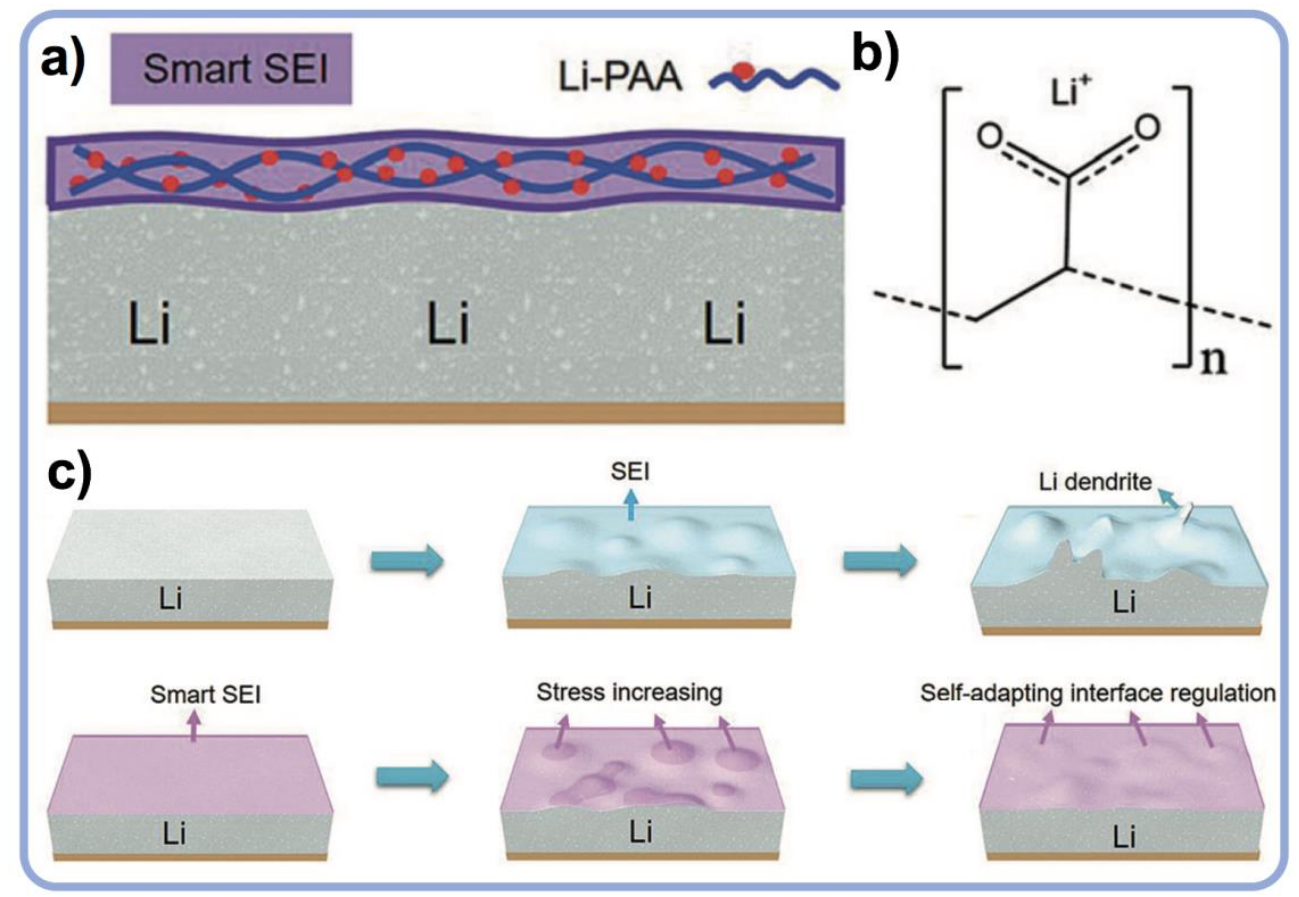

Figure 14. Schematic illustration of a) LiPAA SEI; b) structure of LiPAA polymer; and c) Li dendrites growth on Li metal without and with the protection of LiPAA.[153]

Apart from the higher mechanical strength, a self-adapting ability to the volume changes has also been integrated into the artificial SEIs. Li et.al. proposed a smart SEI film that was composed of lithium polyacrylic acid (LiPAA) prepared by a one-step reaction of Li metal and polyacrylic acid (PAA) (Figure 14a, b).[153] Due to its good stability and elasticity, this polymeric LiPAA-based SEI can spontaneously adjust itself in relation to the volume changes of the Li metal anode during the electrochemical striping/plating process. Therefore, a self-adapting interface regulation was achieved on this SEI film to reduce the cracking during battery operation and thus inhibit the detrimental dendritic $\mathrm{Li}$ formation (Figure 14c), to result in a very stable and reversible $\mathrm{Li}$ 
plating/stripping for $700 \mathrm{~h}$.

\subsection{Ex-situ Formed Artificial SEI Films}

Although the in-situ formed SEI films can provide protection for the Li metal anode, the formation process and the components are generally complicated, the mechanisms are therefore limitedly understood.[154] Moreover, because the microstructure and porosity of these SEI films are normally hard to control, their protection for the Li metal anode is thus difficult to be further optimized.[155] In this regard, the ex-situ artificial SEI films are developed in a more controllable manner with a larger thickness, higher hardness, and better flexibility.[156] Technologies for fabricating the ex-situ SEI films include magnetron sputtering,[157, 158] ALD,[159, 160] Langmuir-Blodgett scooping (LBS),[161] physical transplantation,[162] and so on.[163] In this section, the latest progress in the ex-situ artificial SEI films will be summarized in a sequence of their components.

\subsubsection{Li Ion Contained Ex-situ Artificial SEI Films}

The artificial SEI films should be endowed with high ionic conductivity to realize the fast, stable and uniform transport of $\mathrm{Li}$ ions over the whole electrode surface. Thus, $\mathrm{LiF}, \mathrm{Li}_{3} \mathrm{~N}$, and other $\mathrm{Li}$ compounds, which possess high Li ion conductivity would be suitable components for the SEI films. Studies have also shown that the common components in the in-situ artificial SEI films, such as $\mathrm{Li}_{3} \mathrm{PO}_{4}$, can also be utilized in the fabrication of $e x$-situ artificial SEI films on Li metal anodes due to their very high $\mathrm{Li}$ ion conductivity. 


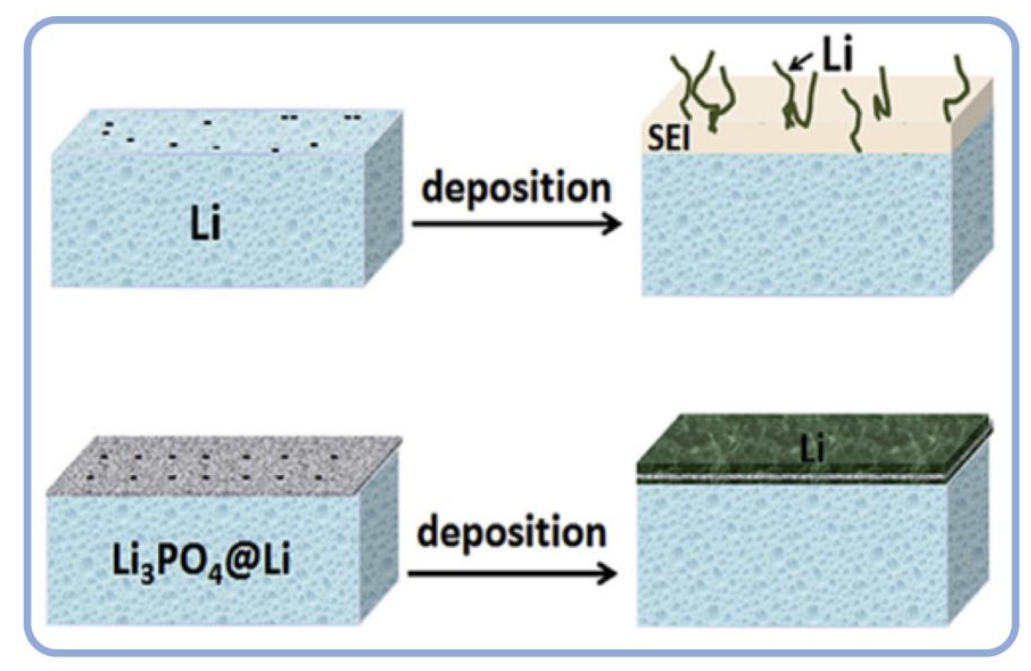

Figure 15. Schematic illustrations of the effect of the $\mathrm{Li}_{3} \mathrm{PO}_{4}$ coating on $\mathrm{Li}$ metal foils in suppressing the Li dendrite formation.[157]

The $\mathrm{Li}_{3} \mathrm{PO}_{4} \mathrm{SEI}$ film can bring in a number of benefits to the cycling behavior of the Li metal anodes. Firstly, the $\mathrm{Li}_{3} \mathrm{PO}_{4}$ film can act as a physical barrier between the $\mathrm{Li}$ metal and the electrolyte, preventing their contact/reaction and thus reducing the loss of Li metal. Secondly, the amorphous $\mathrm{Li}_{3} \mathrm{PO}_{4}$ can lead to a uniform current distribution, resulting in a layer-by-layer $\mathrm{Li}$ deposition manner rather than forming the needle-like dendrites (Figure 15).[157] To prepare a homogeneous and flat $\mathrm{Li}_{3} \mathrm{PO}_{4} \mathrm{SEI}$ film on the $\mathrm{Li}$ metal surface, the magnetron sputtering method is especially convenient, because of its characteristics to achieve a controllable thickness by simply adjusting the sputtering time.[157] Using this method, a homogeneous $\mathrm{Li}_{3} \mathrm{PO}_{4}$ thin film was deposited on a Li metal foil, which could significantly improve the anode performance due to its high chemical stability and the amorphous structure with the advantages of fewer grain boundaries that can provide a high ionic conductivity, a low surface energy, as well as an isotropy nature for a homogeneous current distribution. These merits thus comprehensively induced a uniform deposition of Li metal and the dendritic Li formation can be minimized. 


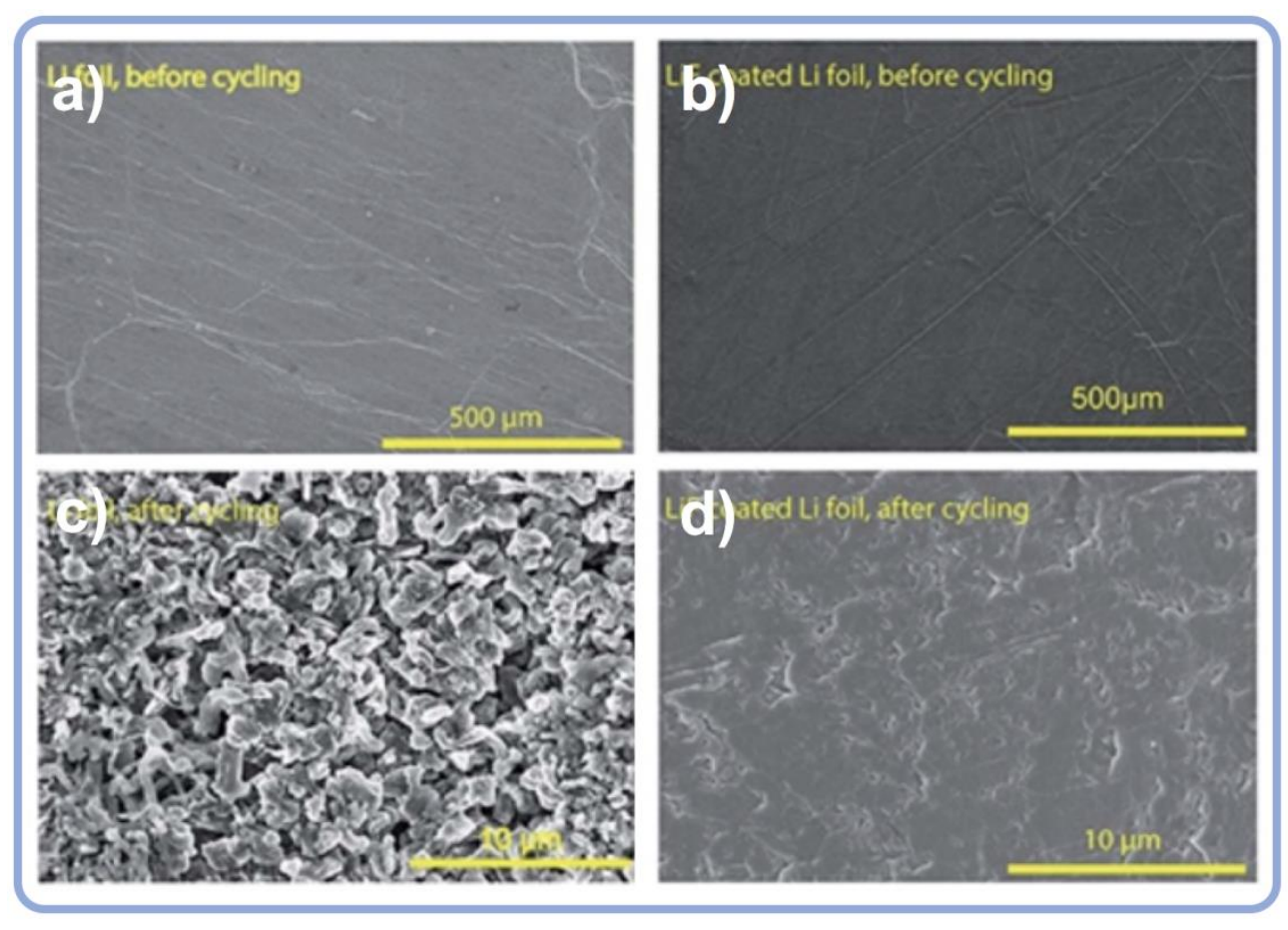

Figure 16. SEM analyses of bare Li metal anode and LiF-coated Li-metal anode generated via the magnetron sputtering.[158]

Using the same method, a LiF artificial SEI film can be generated on the surface of Li metal as well. Compared with other components for SEI, LiF has a lower energy barrier for the diffusion of Li ions and a higher surface energy. As a result, the LiF SEI films usually have a higher Li ion conductivity than the other SEIs. By comparing the bare and the LiF-coated Li metal anode before and after the electrochemical cycles, it is clear that the LiF SEI films can effectively inhibit dendrite formation (Figure 16).[158] Consequently, the assembled $\mathrm{Li}^{2} \mathrm{Li}_{4} \mathrm{Ti}_{5} \mathrm{O}_{12} \mathrm{LMB}$ with the LiF-coated Li metal anode exhibited a high discharge capacity of $135 \mathrm{mAh} \mathrm{g}^{-1}$ over 500 cycles; while the counterpart with a bare Li metal anode died after 350 cycles. 


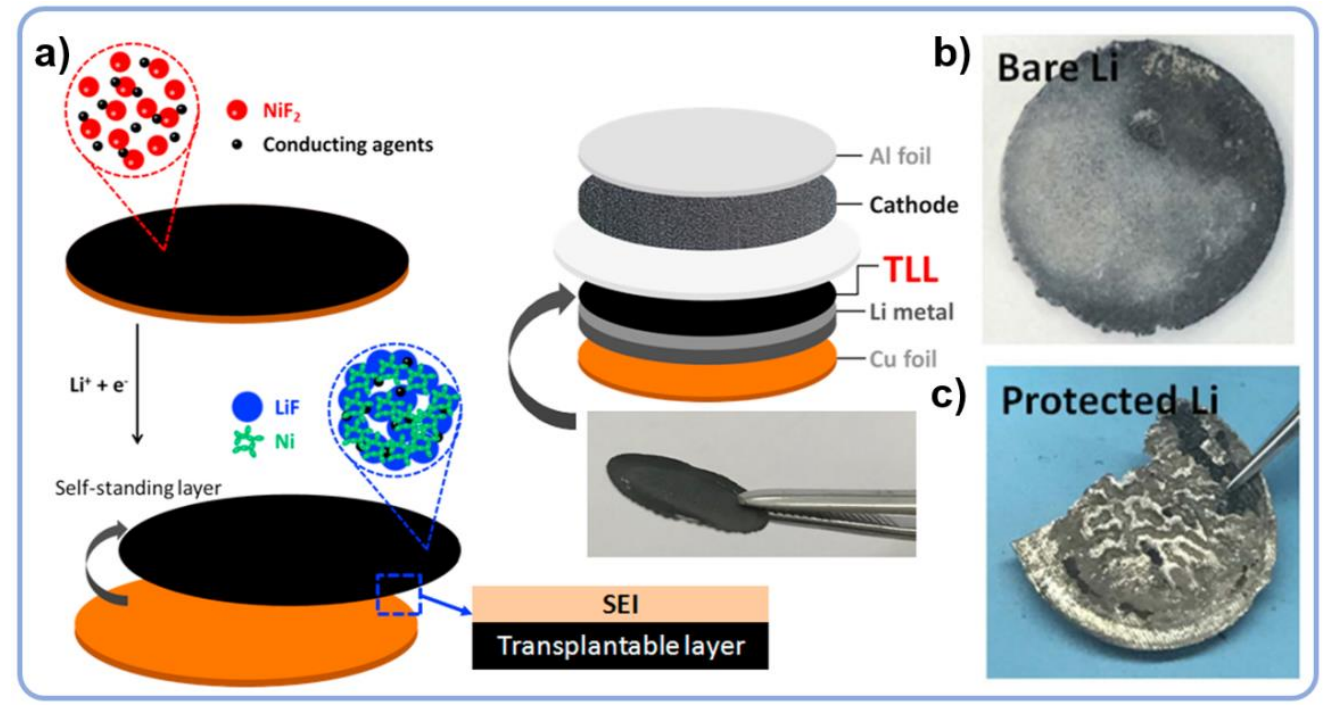

Figure 17. a) Schematic representation of the structural evolution of the nickel fluoride $\left(\mathrm{NiF}_{2}\right)$ during electrochemical reduction the as-formed TLL; and the optic photos of b) the bare Li foil after 650 cycles and c) the TLL protected $\mathrm{Li}$ foil after 1000 cycles in the $\mathrm{Li}-\mathrm{LiFePO}_{4}$ cells.[162]

Such LiF-based SEI films can also be prepared by an electrochemical reduction process. As shown in Figure 17a, a transplantable LiF-rich layer (TLL) composed of nanoscale LiF domains was pre-formed by electrochemically reducing and Li-intercalating of a $\mathrm{NiF}_{2}$-containing and conductive film, which could be directly used to cover the Li metal surface when assembling a LMB.[162] Compared with the SEI films prepared by magnetron sputtering, this TLL not only showed a higher nucleation-potential barrier for $\mathrm{Li}$ metal but also a better transferrable characteristic. In the assembled $\mathrm{Li}-\mathrm{LiFePO}_{4}$ cells, $\mathrm{Li}$ metal with this TLL layer still looked fresh even after 1000 cycles of charge-discharge; while the bare Li foil turned black after 500 cycles, indicating significant side reactions occurred without the protection of this TLL (Figure 17b, c). 


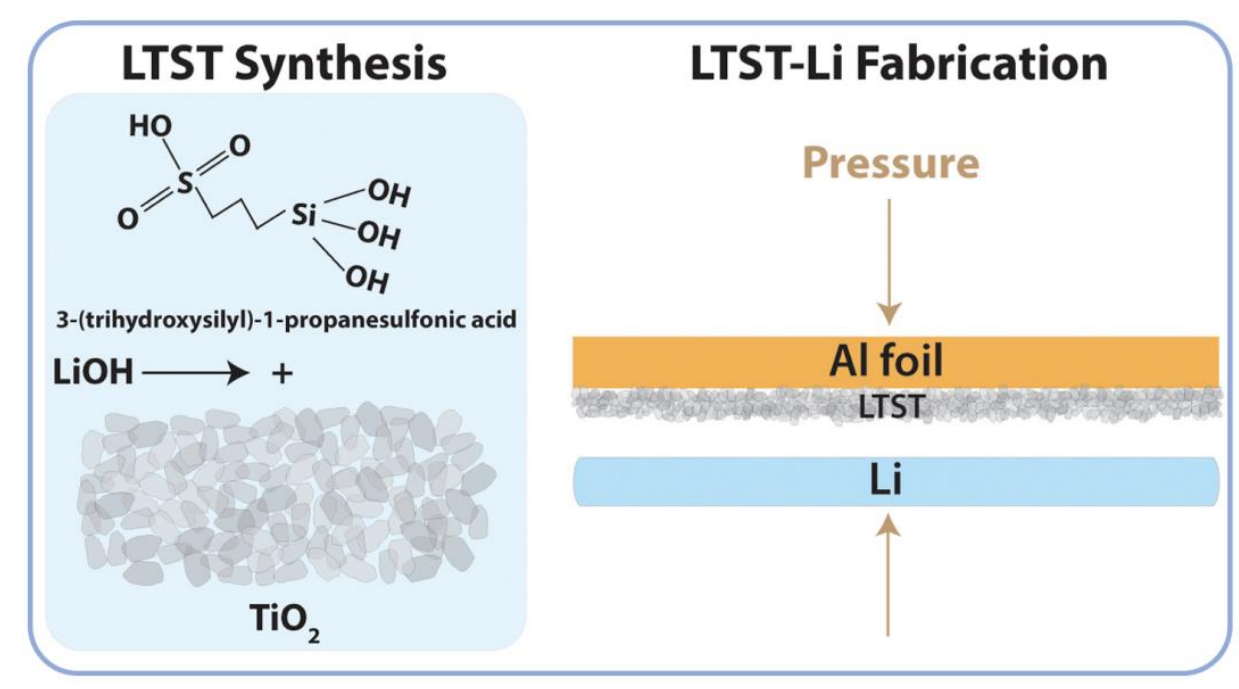

Figure 18. Schematic illustration showing ASEI designs for Li-SB electrodes, and their respective fabrication processes.[161]

Moreover, Langmuir-Blodgett scooping (LBS) is another physical method to directly prepare the ex-situ SEI film, which is able to form the film via a layer-by-layer manner with a highly precise thickness control.[164] For example, a thin artificial SEI film containing the lithium terminated sulfonated titania (LTST) nanoparticles was generated on the surface of Al foil via the LBS method from a suspension of LTST nanoparticles and was subsequently transferred onto Li metal surface by a simple roll-press process and used in a Li-S battery (Figure 18).[161] The SEI film formed with LTST nanoparticles possessed a high Li ionic conductivity and acted as an electrostatical shield to eliminate the agglomeration of the soluble anionic polysulfide components on the anode surface thus stabilizing Li metal surface.[165] Consequently, the coulombic efficiency of the Li-S cells with this LTST coated anode can be up to 90\%.[161]

\subsubsection{Al Ion Contained Ex-situ Artificial SEI Films}

$\mathrm{Al}_{2} \mathrm{O}_{3}$-based SEI film is frequently used as a protecting layer for the Li metal anode, due to its high mechanical strength that can effectively suppress the Li dendrite growth and inhibit the loss of 
the active Li metal. Commonly, $\mathrm{Al}_{2} \mathrm{O}_{3}$ protection layers of a few nanometers thick can be generated by the ALD or sputtering technique yet the process is too complex for practical applications at large scale.[159, 160, 166] To solve this issue, alternative Al-containing SEI layers as well as new methods have been recently reported with a better viability for large scale production.

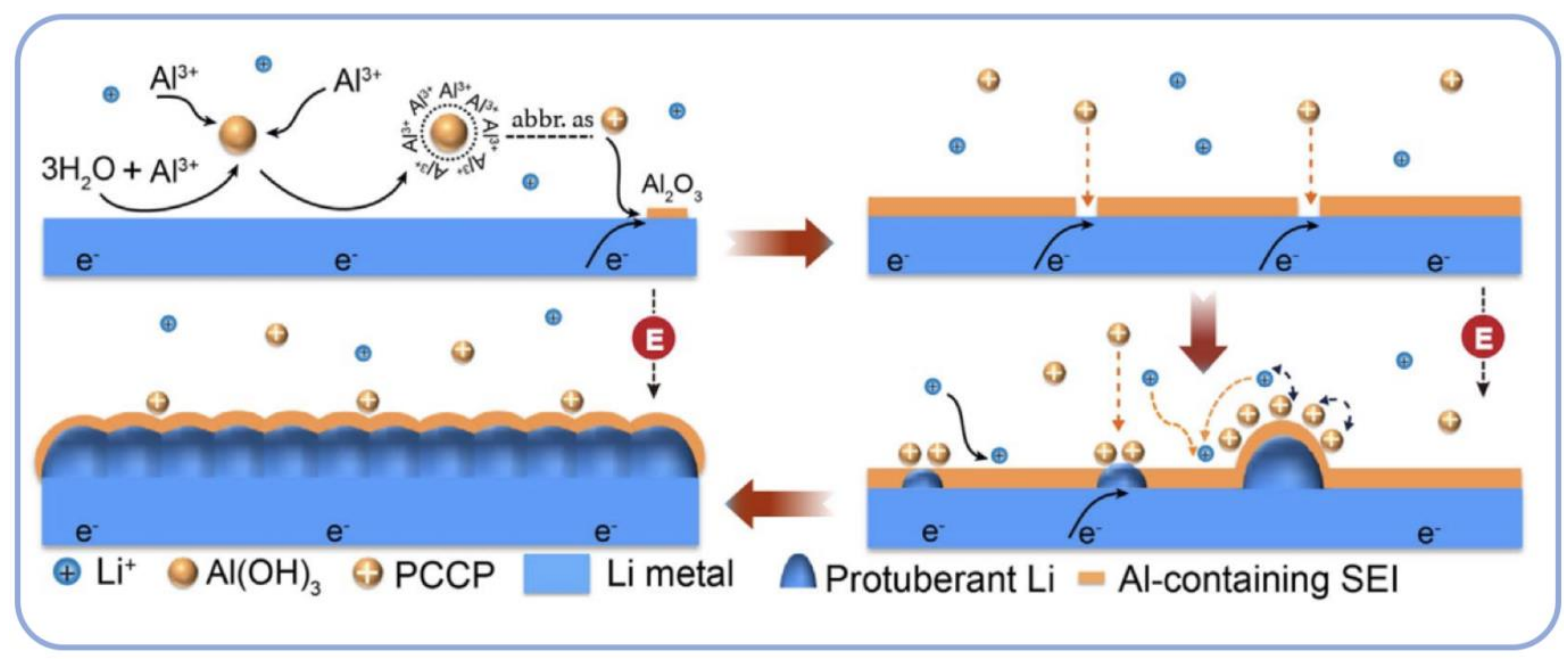

Figure 19. Schematic illustration of the Li plating process with the addition of $\mathrm{AlCl}_{3}$.[167]

Recently, a new approach to generating $\mathrm{Al}_{2} \mathrm{O}_{3}$ SEI artificial film from aluminum halides has been proposed. In this method, $\mathrm{AlCl}_{3}$ was used as an electrolyte additive, which can react with the trace amount of water in the electrolyte and form $\mathrm{Al}(\mathrm{OH})_{3}$ nanoparticles dispersed in the electrolyte (Figure 19).[167] These primary $\mathrm{Al}(\mathrm{OH})_{3}$ particles aggregated on the Li metal surface, further absorbed the rest $\mathrm{Al}^{3+}$ in the electrolyte, and finally formed a continuous $\mathrm{Al}_{2} \mathrm{O}_{3}$ SEI film that fully covered the surface of Li metal anode and enabled a uniform and dendrite-free Li morphology. Due to these excellent characteristics, the rechargeable LMB with this $\mathrm{Al}_{2} \mathrm{O}_{3}$ SEI film can reach a very long life of up to 500 cycles.

\subsubsection{Organic Polymer Contained Ex-situ Artificial SEI Films}

Polymers are electrically insulating and elastic. Therefore, they can be considered as a suitable matrix for artificial SEI films to adapt the large volume changes in electrochemical cycling, upon 
being modified to be able to conduct $\mathrm{Li}$ ions. For this purpose, various polymers have been explored and reported, including polyacetylene,[168] poly(vinylene carbonate-co-acrylonitrile) (P(VC-co-AN)),[169] poly(ethyl $\alpha$-cyanoacrylate) (PECA) [170] etc.

$\operatorname{Poly}((\mathrm{N}-2,2-d i m e t h y l-1,3-d i o x o l a n e-4-m e t h y l)-5-n o r b o r n e n e-e x o-2,3-d i c a r b o x i m i d e)$ is a kind of polymer that has been recently utilized as an implantable protecting layer on the Li metal anode.[170] Figure 20a shows the chemical structure of the polymer that was synthesized by ring-opening metathesis polymerization, and this polymer solution was directly coated on the surface of Li metal to form a protective film after the removal of the solvent. Under this polymeric skin, the Li metal plating/stripping could occur without the dendrite growth, protecting the anode from corrosion with the liquid electrolyte.
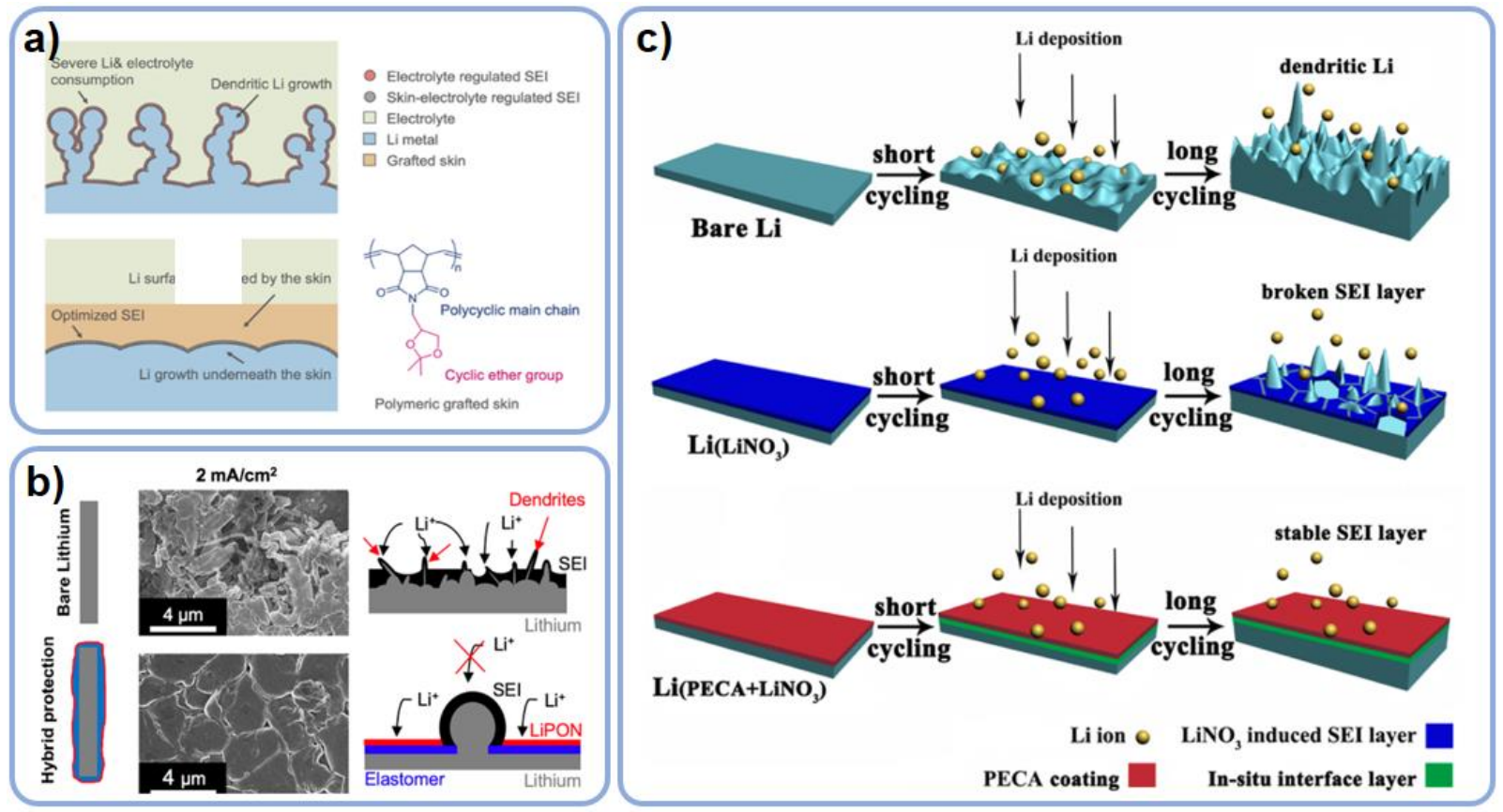

Figure 20. a) Illustration of the different interfacial chemistries of bare Li metal, Li metal with grafted skin in a carbonate electrolyte and chemical structure of the polymer skin, composed of a cyclic ether group (pink) and a polycyclic main chain (blue);[170] b) schematic illustration of the effect of two-layered hybrid artificial SEI film on Li metal and the SEM figures of the Li metal 
batteries with and without the artificial SEI film after cycling;[171] c) schematic illustration of Li deposition on the surface of bare $\mathrm{Li}, \mathrm{Li}\left(\mathrm{LiNO}_{3}\right)$, and $\mathrm{Li}\left(\mathrm{PECA}+\mathrm{LiNO}{ }_{3}\right)$ anode.[170]

The organic-inorganic hybrid ex-situ artificial SEI films, which possess the advantages of both inorganic and polymer layers, have also been developed to protect the Li metal surface. For example, a hybrid ex-situ SEI composed of an outer layer of LiPON and an inner layer of organic elastomeric layer has been designed (Figure 20b).[171] In this hybrid structure, the base elastomeric layer was formed by the polymerization of DOL solution, and the top LiPON layer was then deposited by ALD. In this composite film, the organic elastomeric layer provided mechanical stabilization that could confer mechanical flexibility; while the LiPON layer provided Li-ion conductivity and chemical protection by passivating the $\mathrm{Li}$ metal surface. By applying this LiPON/elastomeric polymer composite coating on the $\mathrm{Li}$ metal anode surface, a good cycling performance up to 300 cycles as well as the successful inhibition of the dendrite growth were achieved. Another type of organic-inorganic hybrid SEI film has also been reported with a high Young's modulus of over $25 \mathrm{GPa}$ to inhibit Li dendrite formation.[170] To prepare it, ethyl $\alpha$-cyanoacrylate (ECA) was used as the monomer, which was reduced by Li metal to form an inorganic nitride layer during cycling. And at the same time, these monomers could also polymerize to poly(ethyl $\alpha$-cyanoacrylate) to form a very smooth organic interface layer on top of Li metal surface. The nitride layer that was generated by the reduction of ECA and $\mathrm{LiNO}_{3}$, which possessed a high mechanical strength to suppress the formation of Li dendrite; while the organic SEI film contributed to a higher stability of the Li anode by separating it from the electrolyte (Figure 20c). These advantages could comprehensively contribute to a very high battery capacity retention up to $93 \%$ after 500 cycles that was much superior to the one with only $\mathrm{LiNO}_{3}$ additive $(43 \%$ after 500 
cycles).

\subsection{Separator Modification}

Considering the overall configuration of the LMBs, dendrite growth from the Li metal anode occurs at the interface between the anode and the separator.[172] Therefore, modifying the separator of LMBs is also an effective way to inhibit the dendritic Li growth, which can be commonly achieved by applying an additional functional coating layer. With this purpose, many organic or inorganic films have been attached onto the separator that can effectively improve the strength of the separator and suppress the Li dendrite growth. These efforts include polydopamine-coated separators,[173] ceramic particles coated separators,[174] and etc.

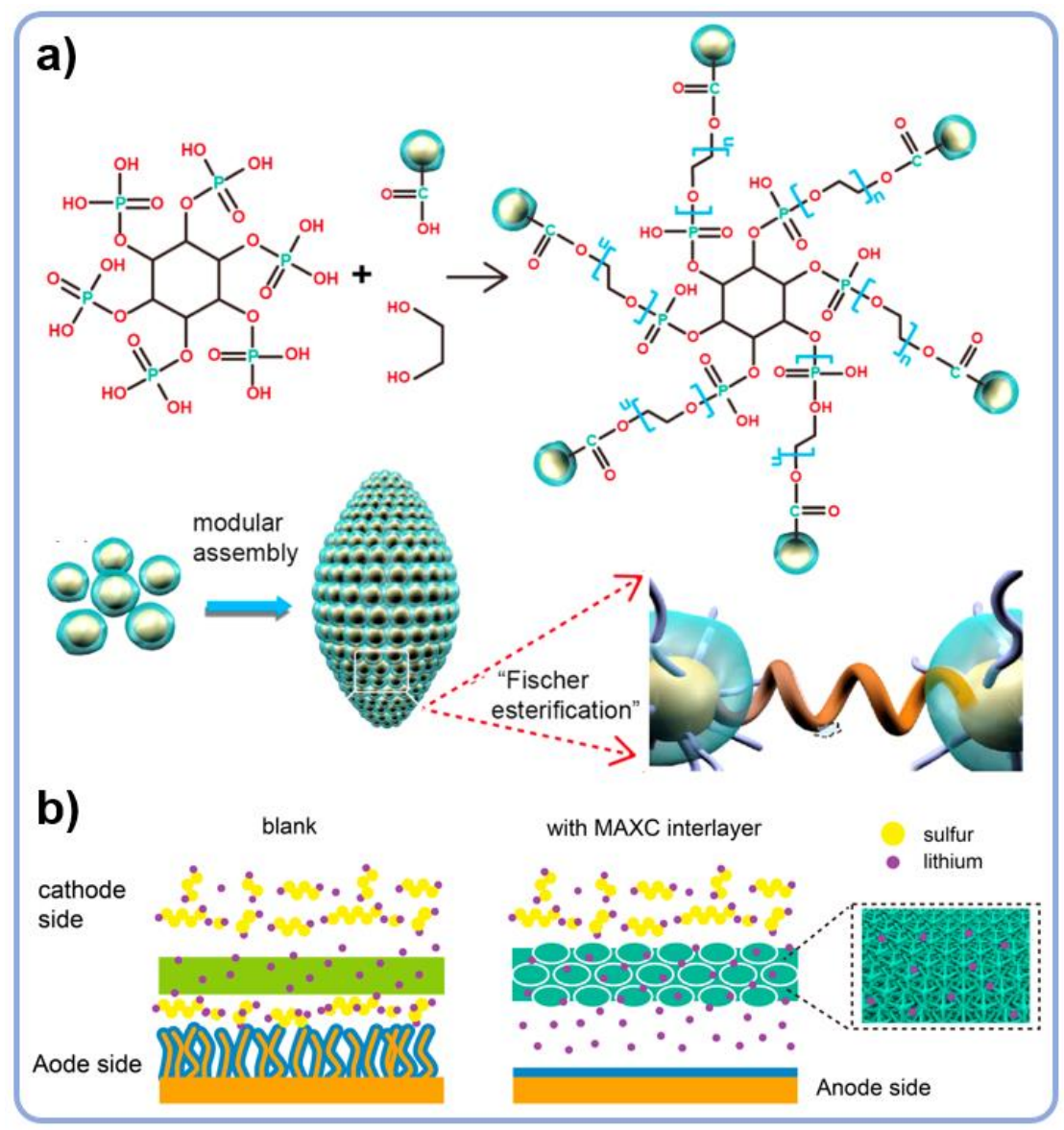

Figure 21. a) Schematic illustration of a molecular reaction om the Vulcan XC72 carbon black nanoparticles to MAXC superstructure and the modular assembly process; and b) schematic 
illustrations of Li-S cell prototypes without and with MAXC interlayer.[175]

Recently, an interesting modularly-assembled interlayer has been synthesized for separator modification for the rechargeable Li-S batteries, which can efficiently trap soluble intermediate polysulfides and keep Li anode intact and stable.[175] This modularly-assembled interlayer (denoted MAXC) was fabricated by agglomerating carbon black nanoparticles into a ellipsoidal superstructure through a double Fischer esterification reaction (Figure 21a). The as-formed MAXC interlayer could provide both the space for storing sufficient electrolyte and efficient immobilization of the soluble polysulfides, in order to prevent them from diffusing to the Li metal anode (Figure 21b). As a result, this MAXC modified separator significantly enhanced the efficiency of Li striping/plating by giving a much higher columbic efficiency of $98.8 \%$ than the bare cell.

Briefly, over the last decade, the interfacial engineering of the Li metal anodes has become a hotspot in the study of improving the stability and cycling life for the liquid-electrolyte LMBs, which can be achieved by the design of artificial SEI films on the surface of Li anode and the modification of the surface of the separators, to effectively suppress Li dendrite growth from different perspectives. Each kind of the in-situ/ex-situ formed SEI films and the modification coatings for the separators has its own particular characteristics that are especially suitable for specific applications, which need to be paid special attention to during their deployment.

\section{Stable Host and Substrate Materials for Li Metals}

As mentioned, the inhomogeneous deposition of $\mathrm{Li}$ ions on the surface of Li metal anode is the main contribution to the dendrite formation, resulting in low coulombic efficiency, shortened life-span, and quick death of LMBs.[176] Therefore, designing stable host and substrate materials to regulate $\mathrm{Li}$ deposition behavior and impede dendrites growth could be possibly effective to deal 
with these issues for Li metal anodes.

Nanoporous frameworks with excellent electrical conductivity and high specific surface area can confine the deposition of $\mathrm{Li}$ in a more homogeneous manner.[177] Benefited from the distinctive pore structure and the large surface area of these nanoscale framework, the electrode local effective current density $(J)$ of Li deposition can be significantly decreased, leading to a larger Sand's time $(\tau$, which means the time for the surface ion concentration on the anode to reduce to 0 , and after it, $\mathrm{Li}$ dendrites start to grow) and thus mitigating the Li dendrite growth.

\subsection{Carbon-based Substrate Materials}

Various carbon materials have been regarded as the suitable matrix to host Li metal because of their good electronic conductivity, high structural versatility, and excellent chemical stability. Carbon materials with various porosity and morphology have been explored for this purpose. For example, $3 \mathrm{D}$ carbon nanotube sponge (CNTS) was coated with an ALD-prepared $\mathrm{Al}_{2} \mathrm{O}_{3}$ layer as an artificial SEI film and was used as an electrode for Li metal deposition in a LMB.[178] Due to the material's high porosity $(>99 \%)$ and large surface area $\left(300-400 \mathrm{~m}^{2} \mathrm{~g}^{-1}\right)$, the obtained CNTS successfully facilitated a very homogenous Li nucleation and effectively suppressed dendrites growth. As a result, a high coulombic efficiency for Li striping/plating of $92.4 \%$ was achieved after 80 cycles, which was superior to the bare $\mathrm{Cu}$ electrode ( $40.2 \%$ at 50 th cycle). In another study, a highly conductive and flexible carbon fiber/carbon foam with a hierarchical structure was also used as a host material for Li metal deposition.[179] On its porous surface, Li metal was deposited in a conformal manner to form a highly stable Li/electrolyte interfaces, which led to high coulombic efficiencies of $98-99 \%$ at a high Li loading of 3-4 $\mathrm{mAh} \mathrm{cm}^{-2}$.

However, common techniques to load $\mathrm{Li}$ metal onto the various substrates, such as 
electrochemical deposition or ALD, are not viable for large-scale production due to their low yield. With the consideration of this, direct impregnation of Li metal into the host material is much more convenient. To achieve this, a carbon nanofiber textile with a surface graphitized layer on individual nanofibers was obtained by thermal treatment, which possessed an enhanced wettability with molten Li compared with the ones treated at lower temperatures.[180] Due to this advantage, this flexible substrate can be simply loaded with $\mathrm{Li}$ by putting them together at a temperature above the melting point of Li (Figure 22a). Even at a high current density of $3 \mathrm{~mA} \mathrm{~cm}^{-2}$, the obtained composite Li metal anode can be still stably cycled for over 100 cycles with a high coulombic efficiency close to $100 \%$.
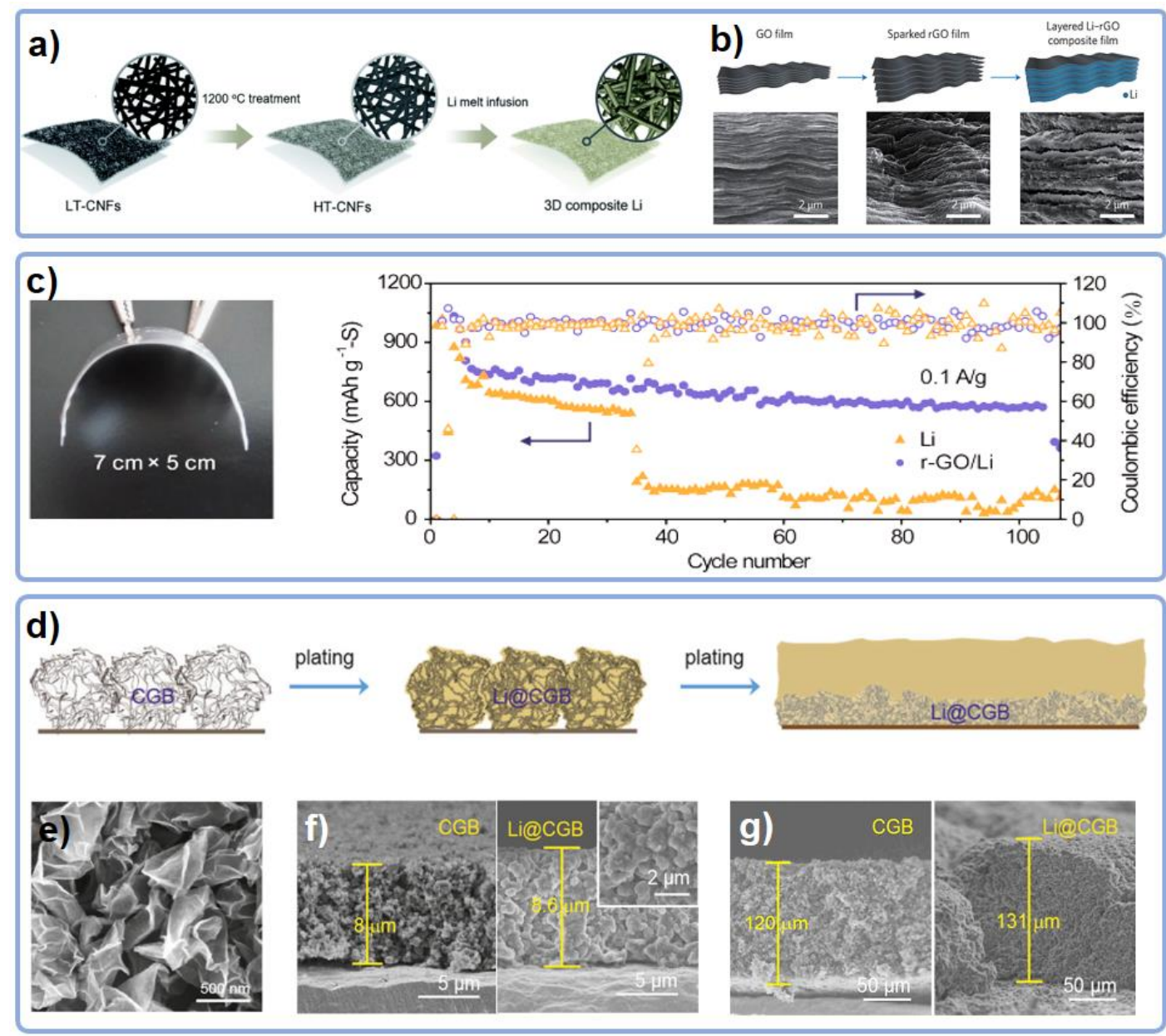

Figure 22. a) Schematic of the 3D composite Li anode formation with HT-CNFs;[180] b) synthesis 
process of Li-rGO composite film and corresponding SEM images;[181] c) photo and cycle performance of $\mathrm{rGO} / \mathrm{Li}$ anode in pouch cell $(7 \times 5 \mathrm{~cm})$ compared with pure $\mathrm{Li}$ anode;[182] d) schematic diagram; SEM images of e) crumpled graphene balls with high supporting for Li plating; f) cross-sectional SEM images of $8 \mu \mathrm{m}$ thick CGB before/after Li deposition of $0.75 \mathrm{mAh} \mathrm{cm}{ }^{-2}$; and g) $120 \mu \mathrm{m}$ thick CGB before/after Li deposition of $10 \mathrm{mAh} \mathrm{cm}^{-2} \cdot[183]$

Apart from the 1D CNTs or carbon fibers, the graphene nanosheet is another ideal matrix for Li deposition, owing to its $2 \mathrm{D}$ features that can effectively provide a large surface area for $\mathrm{Li}$ deposition to reduce the dendrites growth and at the same time achieve a better inter-sheet contact to reduce the interfacial resistance. For example, a nitrogen-doped graphene matrix, with a large pore volume $\left(1.93 \mathrm{~cm}^{3} \mathrm{~g}^{-1}\right)$ and surface area $\left(380.7 \mathrm{~m}^{2} \mathrm{~g}^{-1}\right)$, was designed to accommodate the $\mathrm{Li}$ metal.[178] On this material, the N-doping sites were found to be lithiophilic, which could lead to a more uniform Li metal deposition and thus impede the dendrite formation, resulting in a high coulombic efficiency for Li plating/striping of $98 \%$ for nearly 200 cycles. Innovatively, a more convenient and scalable method was proposed by directly impregnating the molten Li onto the porous and lithiophilic substrates utilizing capillary force.[181] In another example, reduced graphene oxide (rGO) was selected as the host material, and the abundant oxygen-containing surface functional groups could act as a lithiophilic substrate to host Li metal (Figure 22b).[181] Remarkably, by using this host material, a low volume variation (ca. 20\%) between charge and discharge was achieved, together with a good cycling stability and rate capability (ca.110 $\mathrm{mAh} \mathrm{g}^{-1}$ at $4 \mathrm{C}$ and $70 \mathrm{mAh} \mathrm{g}^{-1}$ at $10 \mathrm{C}$ in a full cell with a $\mathrm{LiCoO}_{2}$ cathode). Moreover, graphene can also work as a protective layer for other host materials for Li metal. In this case, a thin and paper-like current collector composed of copper nanowires (CuNWs) covered by a thin graphene layer was 
reported as the host for the dendrite-free Li metal deposition.[184] In this material, the CuNWs with large inter-wire space could accommodate a large amount of Li; while the graphene nanosheets on the outside acted as a high-strength interfacial protective layer to prevent the excessive deposition of Li. As a result, a high coulombic efficiency up to $97 \%$ could be maintained during the 200 cycles test.

By loading $\mathrm{Li}$ metal on certain functional host materials, additional bonus for improved mechanical properties might also be achieved in some cases. For instance, a bendable Li anode was fabricated by integrating Li metal onto a flexible rGO scaffold, which could effectively avoid the crack growth on Li metal upon bending (Figure 22c).[182] This rGO/Li composite anode material was coupled with a CNT/S cathode to form a bendable Li-S pouch cell, with excellent cycling stability for 100 cycles that was far superior to the pristine Li metal foil anode. Recently, Luo and co-workers developed a novel crumpled graphene ball (CGB) to host Li metal (Figure 22d, e).[183] Different from other typical graphene foams or films, this CGB material possessed a much higher resistance to stress and was able to buffer the detrimental volume changes of Li metal during striping and plating. When loaded with a high amount of Li metal up to $10 \mathrm{mAh} \mathrm{cm}^{-2}$, this CGB electrodes still showed a negligible change in volume or any observable dendrites (Figure 22f, $\mathbf{g}$ ). Due to this high structural stability, the CGB/Li composite electrode delivered a very good cycling stability of more than 700 cycles with a high coulombic efficiency of $97.5 \%$, which was much superior to the bare $\mathrm{Cu}$ electrode (below $60 \%$ after 300 cycles).

\subsection{Metal-based Substrate Materials}

Apart from the various carbon substrates, the metal-based matrix materials can also achieve a high affinity to Li metal and are capable to homogenize the Li deposition on their interior/exterior 
surface.[185] A number of studies have been reported on the modification of common $\mathrm{Cu}$ current collectors to better suit the LMBs. For example, on a novel porous $\mathrm{Cu}$ current collector with vertically aligned microchannels (VAMCs), it has been successfully realized the controllable deposition of Li ions into the channels to achieve dendrite-free Li metal anodes (Figure 23a).[186] The microchannels with high aspect ratio divided the bulk current collector into small and narrow regions. Thus, there was no uncontrollably detrimental interact of $\mathrm{Li}^{+}$flux in each single nanochannel, resulting in a relatively uniform Li deposition (Figure 23b, c). The modified anode delivered a great rate and cycle performance with capacity retention of $90 \%$ compared with planar $\mathrm{Cu}$ anode $(80 \%)$ after 100 cycles in a full cell with $\mathrm{LiFePO}_{4}$ cathode. However, the vertically aligned channels are generally fabricated by reactive-ion etching (RIE),[187] which is complicated and might not be suitable for practical applications at a large scale.
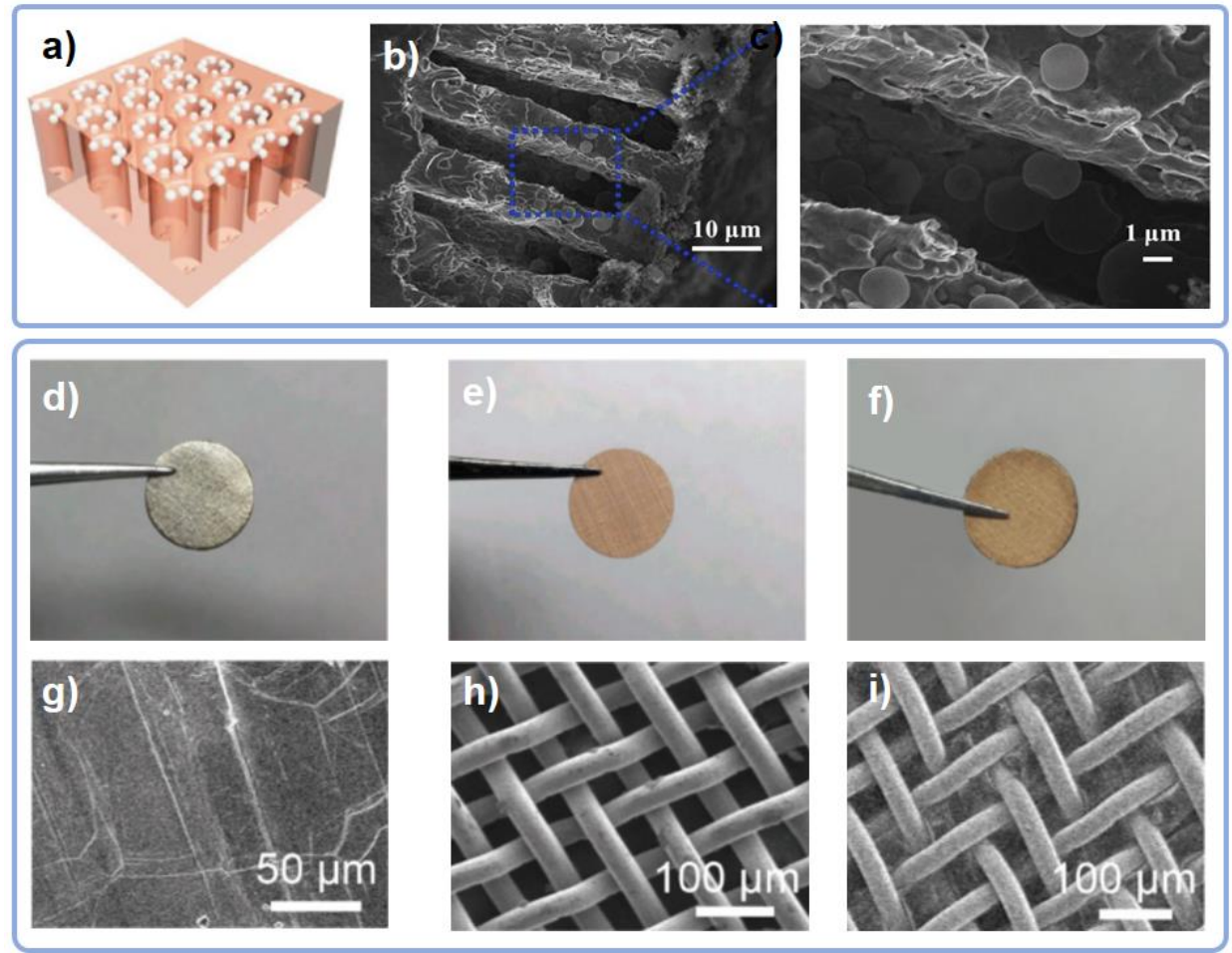

Figure 23. a) Schematic diagram of the preferential deposition of $\mathrm{Li}$ on the modified $\mathrm{Cu}$ current collector; b, c) cross-sectional SEM images of Li deposits on the composite collector at different 
magnifications;[186] and optic images of d) $\mathrm{Li}$ metal, e) $\mathrm{Cu}$ mesh, f) $3 \mathrm{D} \mathrm{Li-metal/Cu} \mathrm{mesh}$ composite, and; g-i) the corresponding SEM images of the above optic images.[188]

In another case, a $\mathrm{Cu}$ mesh/Li-metal composite anode was fabricated by simply embedding the $\mathrm{Cu}$ mesh into Li metal upon mechanical pressure (Figure 23d-i).[188] Compared with the bare Li foil, this 3D $\mathrm{Cu} / \mathrm{Li}$ composite electrode provided a larger surface area and volume to reduce the local current density and accommodate Li deposition during Li plating, thus a higher coulombic efficiency was obtained on this material than the bare Li metal (93.8\% in 100 cycles vs. $30.9 \%$ in 70 cycles). In order to facilitate the large-scale commercial applications, another $3 \mathrm{D} \mathrm{Cu}-\mathrm{Ni}$ current collector for Li metal anodes was recently reported by electrodepositing a porous $\mathrm{Ni}$ coating on $\mathrm{Cu}$ substrate via a one-step, rapid hydrogen bubble dynamic template (HBDT) electrodeposition technique (Figure 24a).[189] The honeycomb-like and porous Ni layer (overall porosity of $81.7 \%$ ) could fully accommodate Li deposition in its abundant pore structure, confining the volume expansion of electrode and significantly enhancing the cycling performance of Li plating/stripping (beyond 300 cycles at $1.0 \mathrm{~mA} \mathrm{~cm}{ }^{-2}$ with an aerial capacity of $0.5 \mathrm{mAh} \mathrm{cm}^{-2}$ ).

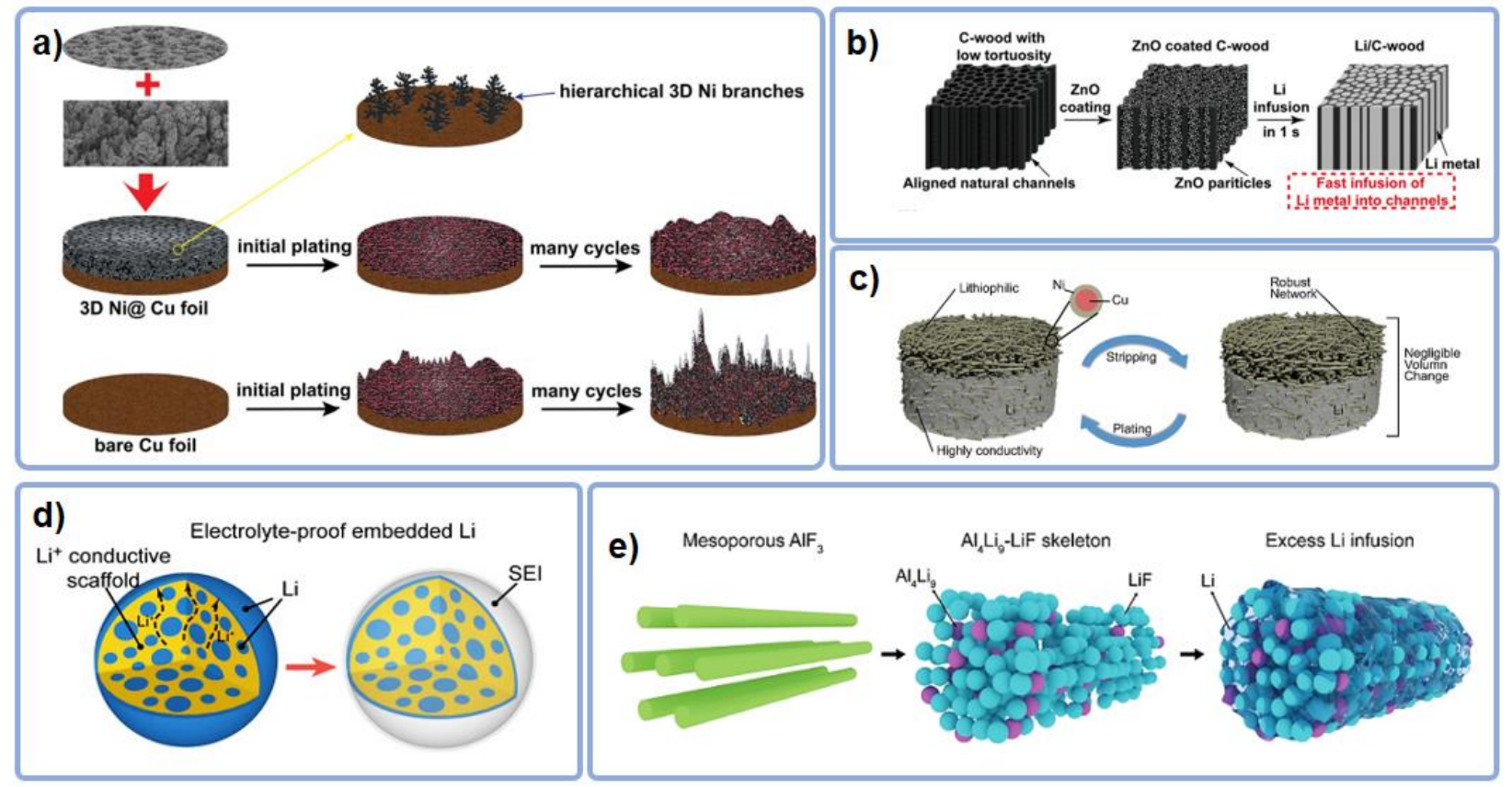


Figure 24. a) Comparison of the Li plating process on 3D Ni-Cu current collector and planar $\mathrm{Cu}$ foil;[189] b) schematic of $\mathrm{Li} / \mathrm{C}$-wood composite design with fast infusion of Li metal into channels;[190] c) design of porous $\mathrm{Li}-\mathrm{Cu}-\mathrm{Ni}$ anode to confine the volume change of electrode;[191] d) schematic of electrolyte-proof architecture;[192] and e) schematic illustration of mesoporous $\mathrm{AlF}_{3}$ framework, $\mathrm{Al}_{4} \mathrm{Li}_{9}-\mathrm{LiF}$ skeleton and LAFN.[193]

Pre-storing Li in host materials can effectively compensate the Li consumption resulted from the simultaneous decomposition of electrolytes. However, in the commonly used electroplating approach by packaging the host material and bare Li foil into a half cell, it is difficult to control the actual deposition behavior of Li. Moreover, the tedious and time-consuming assembling and disassembling process of the half cells to obtain and clean the $\mathrm{Li} /$ matrix composite severely limits its practical application.

In this case, Cui's group pioneered a melting strategy for infusing molten $\mathrm{Li}$ into a porous carbon fiber network matrix coated by a thin lithiophilic silicon layer, which had a very high wettability between matrix and Li metal.[185] Based on this achievement, a fabric polyimide (PI) matrix with ALD-coated $\mathrm{ZnO}$ layer on the PI fibers was also fabricated as a lithiophilic scaffold for molten Li.[181] Notably, the surface of this matrix material was insulating after Li stripping and thus the infused Li became the only electron conductive medium, which avoided the direct deposition of $\mathrm{Li}$ on the outer surface of the PI matrix and successfully confined the undesirable volume expansion during cycling. Similarly, carbonized-wood (C-wood) coated with a thin $\mathrm{ZnO}$ layer on the inner channel walls was fabricated to be a composite $\mathrm{Li} / \mathrm{C}$-wood anode by pre-infusing Li metal into its channels (Figure 24b),[190] and the Li stripping/plating process preferentially occurred in the channels and volume change of the electrode was effectively confined. As a result, the local current 
density was effectively minimized due to the unique channel structure and Li dendrite growth was mitigated. Similarly, such $\mathrm{ZnO} / \mathrm{C}$ composite can also be directly fabricated by annealing a precursor containing both $\mathrm{C}$ and $\mathrm{Zn}$, which achieved a similar enhancement effect.[194]

Nevertheless, these lithiophilic coating layers, especially the metal oxide ones, on these scaffolds would unavoidably increase the internal resistance. Therefore, searching appropriate matrix materials that have a conductive coating or even free of coating layers for Li infusion is necessary. With this aim, a Ni shell was coated on the surface of CuNWs by a very facile electrochemical deposition method, which also achieved a good affinity between $\mathrm{Cu}-\mathrm{Ni}$ nanowires and molten $\mathrm{Li}$ (Figure 24c).[191] Moreover, this $\mathrm{Cu}-\mathrm{Ni}$ scaffold had favorable conductivity and mechanical stability to accommodate the Li plating/stripping and maintained the dimension of anode during cycling. In a full cell using this $\mathrm{Li}-\mathrm{Cu}-\mathrm{Ni}$ anode and $\mathrm{LiCoO}_{2}$ cathode, a high rate capability (90 $\mathrm{mAh} \mathrm{g}^{-1}$ at $5 \mathrm{C}$ ) and cycle stability for 250 cycles were attained. Apart from coating Ni on CuNWs, the commercial Nil foam could directly serve as a stable host for Li infusion by simply dipping Ni foam into molten Li.[195] On the one hand, the porous nature of $\mathrm{Ni}$ foam provided abundant surface area for Li stripping/plating at a low local current density. On the other hand, the good wettability of $\mathrm{Ni}$ with molten Li greatly facilitated the molten Li infusion process. Moreover, the superior electric and ionic conductivity of $\mathrm{Ni}$ foam was also favorable for the rapid ion and electron transport during cycling. As a result of these many advantages, the Li/Ni foam composite electrode displayed a specific capacity of $125 \mathrm{mAh} \mathrm{g}^{-1}$ at $0.5 \mathrm{C}$ with coulombic efficiency of $90 \%$ for 100 cycles, much superior to the bare Li foil anode (30 $\mathrm{mAh} \mathrm{g}^{-1}$ with coulombic efficiency of $\left.72 \%\right)$, confirming an efficient utilization of active Li specie in this composite anode.

Apart from lowing the local current density of Li plating as was discussed above, another 
solution to prevent the $\mathrm{Li}$ dendrite growth or other side reactions, as mentioned in the previous sections, is to isolate the $\mathrm{Li}$ metal from contacting the liquid electrolytes. This principle is also transferrable to the design of the host matrices for $\mathrm{Li}$ metals. For example, a novel $\mathrm{Li}_{\mathrm{x}} \mathrm{Si}_{-}-\mathrm{Li}_{2} \mathrm{O}$ material was synthesized with over stoichiometric Li embedded into SiO (Figure 24d).[192] In this architecture, the active Li domains were dominantly wrapped inside the matrix without direct contact with the electrolyte, which enabled a highly stable and safe Li storage even at high currents. Similarly, another stable $\mathrm{Li} / \mathrm{Al}_{4} \mathrm{Li}_{9}-\mathrm{LiF}$ nanocomposite (LAFN) host was prepared by a one-step "overlithiation" method of mesoporous $\mathrm{AlF}_{3}$ substrate (Figure 24e).[193] The LiF layer generated from this lithiation process could effectively protect the $\mathrm{Li}$ metal and facilitate a uniform Li ion diffusion to hinder the dendrite formation. As a result, this LAFN composite anode could maintain a superior stability in a symmetric cell even at an ultrahigh current density of $20 \mathrm{~mA} \mathrm{~cm}$ for more than 100 cycles, greatly outperforming the conventional Li foil that failed rapidly in less than 20 cycles, giving this material a strong potential for practical high rate LMB applications. 

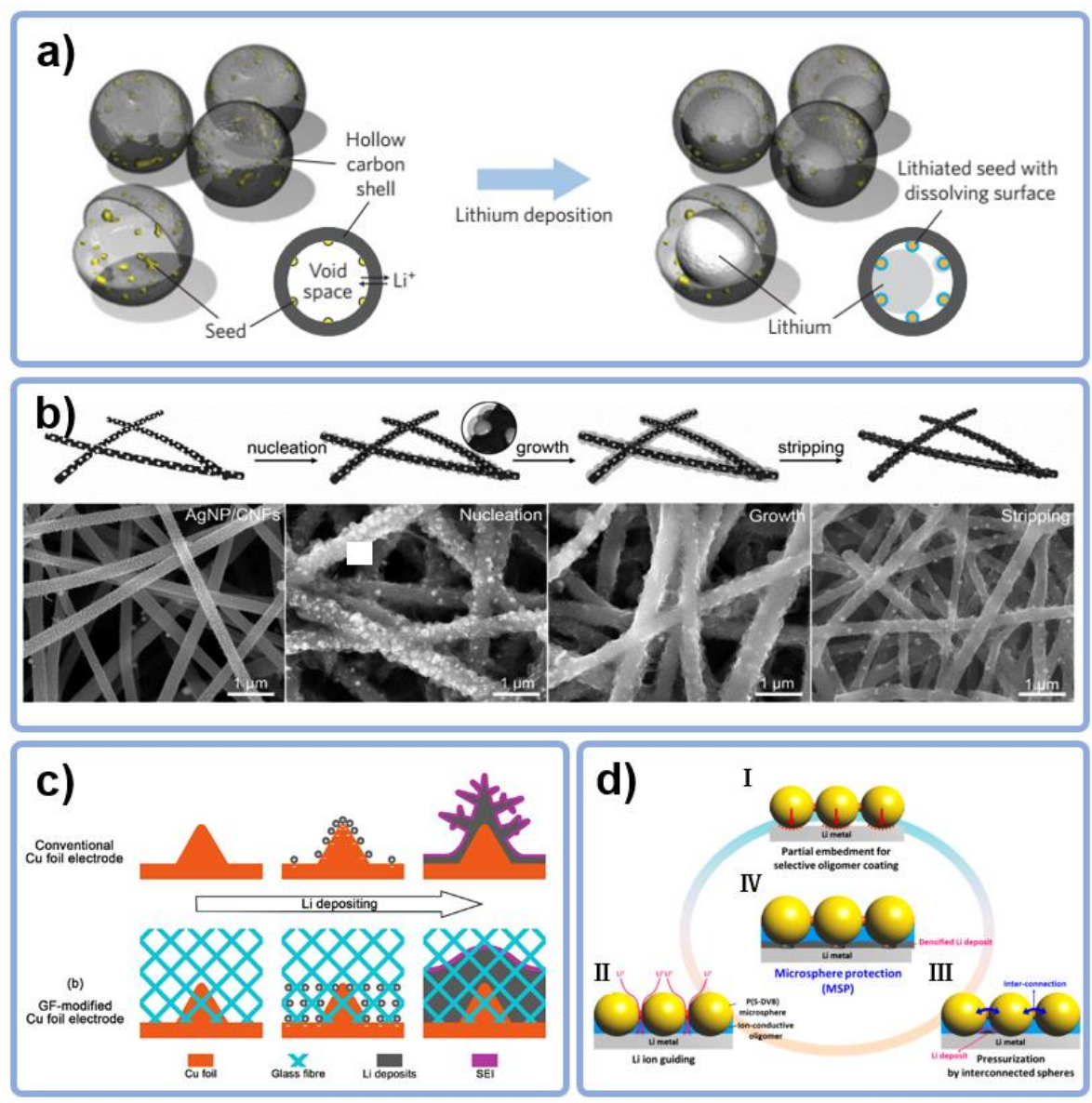

Figure 25. a) Schematic of Li metal nanocapsules;[196] b) schematics of Li nucleation and growth process with AgNP/CNFs seeds and corresponding SEM images;[197] c) schematics of Li plating on 2D Cu foil and GF-modified electrode;[198] and d) illustration of the mechanism of microsphere protection: I) selective oligomer coating induced by embedded microspheres, II) guided Li ions to preferential deposition on the oligomer coating, III) dendrites pressurization by interconnected microspheres, IV) enhanced Li metal anode by MSP.[199]

As discussed above, the various porous carbon nanomaterials could serve as stable hosts for Li metal due to the chemical stability and variability of their structures. Moreover, some metallic substrates (e.g. Ni) have shown good affinity with $\mathrm{Li}$ and can thus effectively attract molten $\mathrm{Li}$ metal with a high affinity. From this end, dispersing these Li-affinitive species on the carbon matrix may also realize selective deposition of Li metal onto these hosts. Cui and co-workers discovered 
that some materials (e.g. Au nanoparticles) possessed zero overpotential of Li nucleation and could serve as heterogeneous seeds to guide Li to selectively nucleate on them.[196] Using hollow carbon nanospheres to host the $\mathrm{Au}$ seeds, $\mathrm{Li}$ deposition can be reversible and preferentially carried out inside the sphere without forming large Li dendrites (Figure 25a), which resulted in a high coulombic efficiency of $98 \%$ over 300 cycles. In another study, ultrafine silver nanoparticles (AgNPs) were anchored uniformly on carbon nanofibers (CNFs) by a rapid high-temperature treatment, which showed a similar effect due to the high tendency of Ag to alloy with Li metal (Figure 25b).[197]

\subsection{Non-conductive Host Materials for Li Metal}

Non-conductive substrate materials, with a high content of polar functional groups (e.g. Si-O, $\mathrm{O}-\mathrm{H}, \mathrm{C} \equiv \mathrm{N}$ ), can also effectively control the deposition of Li. On these materials, the polarized functional groups act as the adhesion sites to adsorb the $\mathrm{Li}$ ions in electrolyte and initiate the subsequent Li plating on these spots. As a result, the distribution of $\mathrm{Li}$ ions is controlled by the dispersion uniformity of these functional groups and dendrites growth can be minimized.[177]

Based on this mechanism, a 3D glass fiber (GF) framework has been proposed for a dendrite-free Li anode (Figure 25c).[198] In this case, GFs were covered on a 2D Cu foil current collector to compensate the curvature-induced and inhomogeneous $\mathrm{Li}$ ion deposition on the bulges of the $\mathrm{Cu}$ foil surface, in order to prevent accumulation of $\mathrm{Li}$ ions and the associated possible dendrites formation.[200] In another study, the typical non-conductive scaffold of oxidized polyacrylonitrile (PAN) nanofiber was selected because of its surface polar groups with good affinity to the electrolytes.[201, 202] Specifically, a free-standing PAN-based insulating microfiber (IMF) matrix was fabricated by electrospinning. The stacked micrometer-sized fibers offered high 
surface affinity to Li ions as well as sufficient internal space for Li metal deposition to minimize the volume change of the electrode with different Li loading amounts even as high as $10 \mathrm{mAh}$ $\mathrm{cm}^{-2}$.[201] Moreover, Lang et al. also reported a PAN fiber array on $\mathrm{Cu}$ foam by a low-cost draw spinning method to guide homogeneous deposition of Li metal,[203] which showed negligible volume variation in the full cell test and a high coulombic efficiency of $97.4 \%$ for 250 cycles, much superior to bare $\mathrm{Cu}$ foam.

Compared to the arrays or microfibers, functional microspheres may as well suppress $\mathrm{Li}$ dendrites; and their high rigidity and regular structure could give extra bonus for this task. Recently, a microsphere-protected $\mathrm{Li}$ metal anode was reported using polystyrene (PS) microspheres copolymerized by divinylbenzene (DVB).[199] As shown in Figure 25d, the regularly arrayed microspheres generated an oligomer coating in the selected areas, guiding a preferential $\mathrm{Li}^{+}$ deposition on the composite anode. As a result, the full cell with such Li metal anode delivered an excellent cycling stability for 190 cycles with $90 \%$ retention of initial specific capacity.

In summary, the purpose of designing porous host materials for $\mathrm{Li}$ metal anodes is mainly reflected in three aspects: i) supplying adequate surface areas for Li ions deposition; ii) minimizing the local current density to alleviate dendrites growth; and iii) providing a strong integration with the $\mathrm{Li}$ ions in the electrolytes to guide the $\mathrm{Li}$ plating. Therefore, seeking appropriate methods to simultaneously obtain these aforementioned functions could be a very attractive and effective way to achieve a Li metal anode with a satisfactory safety and reversibility.

\section{Conclusion and Perspective}

As the core component for the next generation high-energy LMBs, Li metal anodes have attracted a rapidly growing amount of attention due to their exceptional advantages, including low 
density, high theoretical capacity, and low electrochemical potential. However, the high reactivity and spontaneous dendritic Li formation associated with the traditional organic electrolytes are still essential.

With the advance of novel characterization technologies (e.g. Cryo-SEM), deeper understandings about the mechanism of dendrites formation and the interfacial evolution during the operation of LMB have been obtained. Accordingly, numerous efforts have been devoted in the design of novel functional electrolytes, in the modification for a more stable and reliable interface, and in the construction of a composite Li anode.

Although selecting appropriate solutes and functional additives for liquid electrolytes have significantly improved interfacial stability and coulombic efficiency, the possible leakage and flammability nature of liquid electrolytes may still cause serious safety concerns on the LMBs. On the other hand, the solid-state electrolytes have a high mechanical strength that can effectively suppress the dendritic Li formation, especially for inorganic/polymer hybrid SSEs, which are able to simultaneously achieve both high ionic conductivity and mechanical strength/flexibility. However, the poor interfacial wettability and non-negligible resistance between electrolyte and $\mathrm{Li}$ metal require further investigation.

For the interface modification, forming artificial SEI film by in-situ/ex-situ routes and modifying the separator can effectively suppress the Li dendrite. To design artificial SEI films with suitable mechanical strength and chemical stability, the inorganic and organic hybrid SEI films can be a good choice and the possible research direction in the further. In addition to the artificial SEI films, modifying the separator with various functional polymers or ceramic particles is also an important method to improve the stability and the security of the LMBs. 
Constructing porous and stable frameworks to form $3 \mathrm{D}$ composite $\mathrm{Li}$ anodes has been a promising strategy for high performance LMBs in recent years. The nano-/micro-structures serve as stable hosts for Li striping/plating, successfully mitigating the dendrites growth as well as minimizing the volumetric variation of $\mathrm{Li}$ anode. Therefore, exploration of more facile and economical methods to prepare these ideal Li metal anode host are required to be with higher scalability and economical viability so as to be better utilized in LMBs.

Despite the remarkable progress in the last few decades in the development of Li metal anodes for the many types of LMBs, however, a number of remaining challenges still exist at present and continuous explorations are still necessary for the practically usable Li metal anodes. In the many above-summarized strategies, we believe that modification of solid state electrolytes and the corresponding design of novel battery configurations as well as the rational design of the stable host materials for Li metal are two very promising directions that could possibly mitigate these issues in the future. Moreover, the combination of different approaches for commercial application of LMBs is imperative.

\section{Acknowledgements}

This work was supported by Australian Research Council (ARC) through Discovery Early Career Researcher Award (DECRA, No. DE170100871) program, the National Natural Science Foundation of China (No. 51072130 and 51502045), Guangzhou Science and Technology Program (2014Y2-00173), Guangdong Science and Technology Program(2015B020215002), and the open fund of Guangdong Key Laboratory of Membrane Materials and Membrane Separation (No. GIAT2016032101).

\section{Reference}

1. Dunn, B., H. Kamath, and J.M. Tarascon, Electrical Energy Storage for the Grid: A Battery of Choices. Science, 2011. 334(6058): p. 928-935.

2. Evarts, E.C., Lithium Batteries To the Limits of Lithium. Nature, 2015. 526(7575): p. S93-S95.

3. Guo, Y.P., H.Q. Li, and T.Y. Zhai, Reviving Lithium-Metal Anodes for Next-Generation 
High-Energy Batteries. Advanced Materials, 2017. 29(29).

4. Lei, W., et al., Novel Surface Coating Strategies for Better Battery Materials. Surface Innovations, 2018. 6(1-2): p. 13-18.

5. Xu, W., et al., Lithium Metal Anodes for Rechargeable Batteries. Energy \& Environmental Science, 2014. 7(2): p. 513-537.

6. Whittingham, M.S., History, Evolution, and Future Status of Energy Storage. Proceedings of the Leee, 2012. 100: p. 1518-1534.

7. Bruce, P.G., et al., $\mathrm{Li}_{-} \mathrm{O}_{2}$ and Li-S Batteries with High Energy Storage. Nature Materials, 2012. 11(1): p. 19-29.

8. Liang, J., et al., Carbon Materials for Li-S Batteries: Functional Evolution and Performance Improvement. Energy Storage Materials, 2016. 2: p. 76-106.

9. Shen, X., et al., Beyond Lithium Ion Batteries: Higher Energy Density Battery Systems Based on Lithium Metal Anodes. Energy Storage Materials, 2018. 12: p. 161-175.

10. Kong, L., et al., Review of Nanostructured Current Collectors in Lithium-Sulfur Batteries. Nano Research, 2017. 10(12): p. 4027-4054.

11. Whittingham, M.S., Electrical Energy-Storage and Intercalation Chemistry. Science, 1976. 192(4244): p. 1126.

12. Harry, K.J., et al., Detection of Subsurface Structures underneath Dendrites Formed on Cycled Lithium Metal Electrodes. Nature Materials, 2014. 13(1): p. 69-73.

13. Yang, C.P., et al., Protected Lithium-Metal Anodes in Batteries: From Liquid to Solid. Advanced Materials, 2017. 29(36).

14. J. Zhang, W.X., W. A. Henderson, Lithium Metal Anodes and Rechargeable Lithium Metal Batteries. 2016: Springer.

15. Zhang, K., et al., Recent Developments of the Lithium Metal Anode for Rechargeable Non-Aqueous Batteries. Advanced Energy Materials, 2016. 6(20).

16. Xu, R., et al., Artificial Soft-Rigid Protective Layer for Dendrite-Free Lithium Metal Anode. Advanced Functional Materials: p. 1705838.

17. Aurbach, D., et al., A Short Review of Failure Mechanisms of Lithium Metal and Lithiated Graphite Anodes in Liquid Electrolyte Solutions. Solid State Ionics, 2002. 148(3-4): p. 405-416.

18. Cheng, X.B., et al., Toward Safe Lithium Metal Anode in Rechargeable Batteries: A Review. Chemical Reviews, 2017. 117(15): p. 10403-10473.

19. Sun, C.W., et al., Recent Advances in All-Solid-State Rechargeable Lithium Batteries. Nano Energy, 2017. 33: p. 363-386.

20. D., A., Identification of Surface Films Formed on Lithium in Propylene Carbonate Solutions. Journal of the Electrochemical Society, 1987. 134(7): p. 1611-1620.

21. Xu, K., Nonaqueous Liquid Electrolytes for Lithium-Based Rechargeable Batteries. Chemical Reviews, 2004. 104(10): p. 4303-4417.

22. Gao, Y., R. Yi, and Y.C. Li, A General Method of Manipulating Formation, Composition, and Morphology of Solid-Electrolyte Interphases for Stable Li-Alloy Anodes. Journal of the American Chemical Society, 2017. 129(48):p. 28359-17367

23. Chen, X., et al., Ion-Solvent Complexes Promote Gas Evolution from Electrolytes on a Sodium Metal Anode. Angewandte Chemie International Edition, 2018. 57(3): p. 734-737.

24. Cohen, Y.S., Y. Cohen, and D. Aurbach, Micromorphological Studies of LIthium Electrodes in Alkyl Carbonate Solutions Using In Situ Atomic Force Microscopy. Journal of Physical Chemistry B, 2000. 104(51): p. 12282-12291. 
25. Qi, Y., et al., Threefold Increase in the Young's Modulus of Graphite Negative Electrode during Lithium Intercalation. Journal of the Electrochemical Society, 2010. 157(5): p. A558-A566.

26. de las Casas, C. and W.Z. Li, A Review of Application of Carbon Nanotubes for Lithium Ion Battery Anode Material. Journal of Power Sources, 2012. 208: p. 74-85.

27. Goriparti, S., et al., Review on Recent Progress of Nanostructured Anode Materials for Li-Ion Batteries. Journal of Power Sources, 2014. 257: p. 421-443.

28. T., O., U. A., and Y. N., Zero-Strain Insertion Material of $\mathrm{Li}_{[}\left[\mathrm{Li}_{1 / 3} \mathrm{Ti}_{5 / 3} \mathrm{Ti}_{5 / 3}\right] \mathrm{O}_{4}$ for Rechargeable Lithium Cells. Journal of the Electrochemical Society, 1995. 142(5): p. 1431-1435.

29. Zhou, X.S., et al., Spin-Coated Silicon Nanoparticle/Graphene Electrode as a Binder-Free Anode for High-Performance Lithium-Ion Batteries. Nano Research, 2012. 5(12): p. 845-853.

30. Choi, N.S., et al., Recent Progress on Polymeric Binders for Silicon Anodes in Lithium-Ion Batteries. Journal of Electrochemical Science and Technology, 2015. 6(2): p. 35-49.

31. Tian, H., et al., High Capacity Group-IV Elements (Si, Ge, Sn) Based Anodes for Lithium-Ion Batteries. Journal of Materiomics, 2015. 1(3): p. 153-169.

32. Zhao, Y., et al., Significant Impact of 2D Graphene Nanosheets on Large Volume Change Tin-Based Anodes in Lithium-Ion Batteries: A Review. Journal of Power Sources, 2015. 274: p. 869-884.

33. Zhang, F., et al., Effects of the Volume Changes and Elastic-Strain Energies on the Phase Transition in the Li-Sn Battery. Journal of Power Sources, 2016. 330: p. 111-119.

34. Jiang, Y., et al., Electrostatic Spray Deposition of Porous $\mathrm{SnO}_{2} /$ Graphene Anode Films and their Enhanced Lithium-Storage Properties. ACS Applied Materials \& Interfaces, 2012. 4(11): p. 6216-6220.

35. Zhou, W., S. Upreti, and M.S. Whittingham, Electrochemical Performance of Al-Si-Graphite Composite as Anode for Lithium-Ion Batteries. Electrochemistry Communications, 2011. 13(2): p. 158-161.

36. Lin, D.C., Y.Y. Liu, and Y. Cui, Reviving the Lithium Metal Anode for High-Energy Batteries. Nature Nanotechnology, 2017. 12(3): p. 194-206.

37. Brissot, C., et al., Dendritic Growth Mechanisms in Lithium/Polymer Cells. Journal of Power Sources, 1999. 81: p. 925-929.

38. J., C., Electrochemical Aspects of the Generation of Ramified Metallic Electrodeposits. Physical Review A Atomic Molecular \& Optical Physics, 1990. 42(12): p. 7355-7367.

39. Orsini, F., et al., In Situ Scanning Electron Microscopy (SEM) Observation of Interfaces within Plastic Lithium Batteries. Journal of Power Sources, 1998. 76(1): p. 19-29.

40. Stark, J.K., Y. Ding, and P.A. Kohl, Dendrite-Free Electrodeposition and Reoxidation of Lithium-Sodium Alloy for Metal-Anode Battery. Journal of the Electrochemical Society, 2011. 158(10): p. A1100-A1105.

41. Morigaki, K., Analysis of the Interface between Lithium and Organic Electrolyte Solution. Journal of Power Sources, 2002. 104(1): p. 13-23.

42. Morigaki, K. and A. Ohta, Analysis of the Surface of Lithium in Organic Electrolyte by Atomic Force Microscopy, Fourier Transform Infrared Spectroscopy and Scanning Auger Electron Microscopy. Journal of Power Sources, 1998. 76(2): p. 159-166.

43. Ghassemi, H., et al., Real-Time Observation of Lithium Fibers Growth inside a Nanoscale Lithium-Ion Battery. Applied Physics Letters, 2011. 99(12).

44. Liu, X.H., et al., Lithium Fiber Growth on the Anode in a Nanowire Lithium Ion Battery during Charging. Applied Physics Letters, 2011. 98(18). 
45. Li, Y., et al., Atomic Structure of Sensitive Battery Materials and Interfaces Revealed by Cryo-Electron Microscopy. Science, 2017: p. 506-510.

46. Peled, E., The Electrochemical Behavior of Alkali and Alkaline Earth Metals in Nonaqueous Battery Systems-The Solid Electrolyte Interphase Model. Journal of the Electrochemical Society, 1979. 126: p. 2047-2051.

47. Cheng, X.-B., et al., The Gap Between Long Lifespan Li-S Coin and Pouch Cells: The Importance of Lithium Metal Anode Protection. Energy Storage Materials, 2017. 6: p. 18-25.

48. Chen, X., et al., Towards Stable lithium-Sulfur Batteries: Mechanistic Insights into Electrolyte Decomposition on Lithium Metal Anode. Energy Storage Materials, 2017. 8: p. 194-201.

49. Aurbach, D., et al., Recent Studies of the Lithium-Liquid Electrolyte Interface Electrochemical, Morphological and Spectral Studies of a few Important Systems. Journal of Power Sources, 1995. 54(1): p. 76-84.

50. Rauh, R.D., T.F. Reise, and S.B. Brummer, Efficiencies of Cycling Lithium on a Lithium Substrate in Propylene Carbonate. Journal of the Electrochemical Society, 1978. 125: p. 186-190.

51. Aurbach, D., et al., A Comparative Study of Synthetic Graphite and Li Electrodes in Electrolyte Solutions Based on Ethylene Carbonate-Dimethyl Carbonate Mixtures. Journal of the Electrochemical Society, 1996. 143(12): p. 3809-3820.

52. Koch, V.R. and J.H. Young, The Stability of the Secondary Lithium Electrode in Tetrahydrofuran - Based Electrolytes. Journal of the Electrochemical Society, 1978. 125: p. 1371-1377.

53. Goldman, J.L., et al., Structure - Reactivity Relationships of Methylated Tetrahydrofurans with Lithium. Journal of the Electrochemical Society, 1980. 127: p. 1461-1467.

54. Foos, J.S. and J. McVeigh, Lithium Cycling in Polymethoxymethane Solvents. Journal of the Electrochemical Society, 1983. 130(3): p. 628-630.

55. Koch, V.R., et al., Specular Lithium Deposits from Lithium Hexafluoroarsenate-Diethyl Ether Electrolytes. Journal of the Electrochemical Society, 1982. 129: p. 1-4.

56. Selim, R. and P. Bro, Some Observations on Rechargeable Lithium Electrodes in a Propylene Carbonate Electrolyte. Journal of the Electrochemical Society, 1974. 121: p. 1457-1459.

57. Geronov, Y., et al., Lithium Electrode Morphology during Cycling in Lithium Cells. Journal of the Electrochemical Society, 1988. 135(10): p. 2422-2427.

58. Hayashi, K., et al., Mixed Solvent Electrolyte for High Voltage Lithium Metal Secondary Cells. Electrochimica Acta, 1999. 44(14): p. 2337-2344.

59. Hayashi, K., Y. Nemoto, and S. Tobishima, Dependence of Lithium Metal Secondary Cell Performance on Lithium Cation Solvation State. Journal of Power Sources, 1999. 81: p. 782-785

60. Tobishima, S., et al., Cycling Performance and Safety of Rechargeable Lithium Cells with Binary and Ternary Mixed Solvent Electrolytes. Journal of Applied Electrochemistry, 1999. 29(7): p. 789-796.

61. Tarascon, J.M. and D. Guyomard, New Electrolyte Compositions Stable over the 0 to $5 \mathrm{~V}$ Voltage Range and Compatible with the $\mathrm{Li}_{1+x} \mathrm{Mn}_{2} \mathrm{O}_{4} /$ Carbon Li-Ion Cells. Solid State Ionics, 1994. 69: p. 293-305.

62. Bieker, G., M. Winter, and P. Bieker, Electrochemical In Situ Investigations of SEI and Dendrite Formation on the Lithium Metal Anode. Physical Chemistry Chemical Physics, 2015. 17(14): p. 8670-8679.

63. Peled, E., et al., An Advanced Tool for the Selection of Electrolyte Components for Rechargeable Lithium Batteries. Journal of the Electrochemical Society, 1998. 145: p. 3482-3486. 
64. Ein-Eli, Y., et al., Ethylmethylcarbonate, a Promising Solvent for Li - Ion Rechargeable Batteries. Journal of the Electrochemical Society, 2012. 143: p. L273-L277.

65. Neuhold, S., D.J. Schroeder, and J.T. Vaughey, Effect of Surface Preparation and R-group Size on the Stabilization of Lithium Metal Anodes with Silanes. Journal of Power Sources, 2012. 206: p. 295-300.

66. Takei, Y., et al., Effects of Nonaqueous Electrolyte Solutions Mixed with Carbonate-Modified Siloxane on Charge-Discharge Performance of Negative Electrodes for Secondary Lithium Batteries. Journal of Power Sources, 2013. 228: p. 32-38.

67. Zhou, H., Z. Fang, and J. Li, LiPF 6 and Lithium Difluoro(oxalato)borate/Ethylene Carbonate+Dimethyl Carbonate+Ethyl(methyl)carbonate Electrolyte for $\mathrm{Li}_{4} \mathrm{Ti}_{5} \mathrm{O}_{12}$ Anode. Journal of Power Sources, 2013. 230(5): p. 148-154.

68. Aurbach, D. and Y. Gofer, The Correlation Between Surface Chemistry, Surface Morphology, and Cycling Efficiency of Lithium Electrodes in a few Polar Aprotic Systems. Journal of the Electrochemical Society, 1989. 136: p. 3198-3205.

69. Nanjundiah, C., et al., Electrochemical Stability of $\operatorname{LiMF}_{6}(M=P, A s, S b)$ in Tetrahydrofuran and Sulfolane. Journal of the Electrochemical Society, 1988. 135: p. 2914-2917.

70. Ishikawa, M., et al., Charge/Discharge Characteristics of Carbon Fiber with Graphite Structure in Organic Electrolytes Containing Lithium Trifluoromethanesulfate and Lithium Hexafluorophosphate. Journal of the Electrochemical Society, 1994. 141(5): p. 1105-1108.

71. Zhang, S.S., Role of $\mathrm{LiNO}_{3}$ in Rechargeable Lithium/Sulfur Battery. Electrochimica Acta, 2012. 70: p. 344-348.

72. Zhao, C.-Z., et al., Li $\mathrm{i}_{2} \mathrm{~S}_{5}$ Based Ternary-Salt Electrolyte for Robust Lithium Metal Anode. Energy Storage Materials, 2016. 3: p. 77-84.

73. Aurbach, D., et al., The Study of Electrolyte Solutions Based on Ethylene and Diethyl Carbonates for Rechargeable Li Batteries: II . Graphite Electrodes. Journal of the Electrochemical Society, 1995. 142(9): p. 2882-2890.

74. Ue, M., Mobility and Ionic Association of Lithium Salts in a Propylene Carbonate-Ethyl Methyl Carbonate Mixed Solvent. Journal of the Electrochemical Society, 1995. 142: p. 2577-2581.

75. Tikekar, M.D., L.A. Archer, and D.L. Koch, Stability Analysis of Electrodeposition across a Structured Electrolyte with Immobilized Anions. Journal of the Electrochemical Society, 2014. 161: p. A847-855.

76. Xiong, S., et al., On the Role of Polysulfides for a Stable Solid Electrolyte Interphase on the Lithium Anode Cycled in Lithium-Sulfur Batteries. Journal of Power Sources, 2013. 236: p. 181-187.

77. Aurbach, D., et al., On the Surface Chemical Aspects of Very High Energy Density, Rechargeable Li-Sulfur Batteries. Journal of the Electrochemical Society, 2009. 156: p. A694.

78. Li, W.Y., et al., The Synergetic Effect of Lithium Polysulfide and Lithium Nitrate to Prevent Lithium Dendrite Growth. Nature Communications, 2015. 6.

79. Kita, F., H. Sakata, and A. Kawakami, Electronic Structures and Electrochemical Properties of $\operatorname{LiPF}_{6-n}\left(C F_{3}\right)_{n}$. Journal of Power Sources, 2001. 97: p. 581-583.

80. Aurbach, D., et al., Vinylene Carbonate and Li Salicylatoborate as Additives in $\mathrm{LiPF}_{3}\left(\mathrm{CF}_{2} \mathrm{CF}_{3}\right)_{3}$ Solutions for Rechargeable Li-Ion Batteries. Journal of the Electrochemical Society, 2004. 151(1): p. A23-A30.

81. Xiong, S., et al., Properties of Surface Film on Lithium Anode with $\mathrm{LiNO}_{3}$ as Lithium Salt in Electrolyte Solution for Lithium-Sulfur Batteries. Electrochimica Acta, 2012. 83: p. 78-86. 
82. Choudhury, S., et al., Designer Interphases For the Lithium-Oxygen Electrochemical Cell. Science Advances, 2017. 3(4).

83. Zhang, L., et al., The Synergetic Interaction between $\mathrm{LiNO}_{3}$ and Lithium Polysulfides for Suppressing Shuttle Effect of Lithium-Sulfur Batteries. Energy Storage Materials, 2018. 11: p. 24-29.

84. Cheng, X.-B., et al., Sulfurized Solid Electrolyte Interphases with a Rapid $\mathrm{Li}^{+}$Diffusion on Dendrite-Free Li Metal Anodes. Energy Storage Materials, 2018. 10: p. 199-205.

85. Kim, H., et al., Metallic Anodes for Next Generation Secondary Batteries. Chemical Society Reviews, 2013. 42(23): p. 9011-9034.

86. Lin, D., Y. Liu, and Y. Cui, Reviving the Lithium Metal Anode for High-Energy Batteries. Nature Nanotechnology, 2017. 12(3): p. 194-206.

87. Osaka, T., et al., Surface Characterization of Electrodeposited Lithium Anode with Enhanced Cycleability Obtained by $\mathrm{CO}_{2}$ Addition. Journal of the Electrochemical Society, 1997. 144: p. 1709-1713.

88. Besenhard, J., et al., Inorganic Film-Forming Electrolyte Additives Improving the Cycling Behaviour of Metallic Lithium Electrodes and the Self-Discharge of Carbon-Lithium Electrodes. Journal of Power Sources, 1993. 44: p. 413-420.

89. Koshikawa, H., et al., Effects of Contaminant Water on Coulombic Efficiency of Lithium Deposition/Dissolution Reactions in Tetraglyme-Based Electrolytes. Journal of Power Sources, 2017. 350: p. 73-79.

90. Togasaki, N., T. Momma, and T. Osaka, Enhancement Effect of Trace $\mathrm{H}_{2} \mathrm{O}$ on the Charge-Discharge Cycling Performance of a Li Metal Anode. Journal of Power Sources, 2014. 261: p. 23-27.

91. Ota, H., et al., Characterization of Lithium Electrode in Lithium Imides/Ethylene Carbonate and Cyclic Ether Electrolytes: II. Surface Chemistry Journal of the Electrochemical Society, 2004. 151: p. A437-A446.

92. Lu, Y., Z. Tu, and L.A. Archer, Stable Lithium Electrodeposition in Liquid and Nanoporous Solid Electrolytes. Nature Materials, 2014. 13(10): p. 961-969.

93. Liu, Q.C., et al., Artificial Protection Film on Lithium Metal Anode toward Long-Cycle-Life Lithium-Oxygen Batteries. Advanced Materials, 2015. 27(35): p. 5241-5147.

94. Zhang, X.Q., et al., Fluoroethylene Carbonate Additives to Render Uniform Li Deposits in Lithium Metal Batteries. Advanced Functional Materials, 2017. 27(10).

95. Mogi, R., et al., Effects of Some Organic Additives on Lithium Deposition in Propylene Carbonate. Journal of the Electrochemical Society, 2002. 149(12): p. A1578-A1583.

96. Ding, F., et al., Dendrite-Free Lithium Deposition via Self-Healing Electrostatic Shield Mechanism. Journal of the American Chemical Society, 2013. 135(11): p. 4450-4456.

97. Ding, F., et al., Effects of Cesium Cations in Lithium Deposition via Self-Healing Electrostatic Shield Mechanism. The Journal of Physical Chemistry C, 2014. 118(8): p. 4043-4049.

98. Hu, J.Z., et al., In Situ $7 \mathrm{Li}$ and 133 Cs Nuclear Magnetic Resonance Investigations on the Role of $\mathrm{Cs}^{+}$Additive in Lithium-Metal Deposition Process. Journal of Power Sources, 2016. 304: p. 51-59.

99. Xu, Q., Y. Yang, and H. Shao, Enhanced Cycleability and Dendrite-Free Lithium Deposition by Adding Potassium Ion to the Electrolyte for Lithium Metal Batteries. Electrochimica Acta, 2016. 212: p. $758-766$.

100. Wood, S.M., et al., $K^{+}$Reduces Lithium Dendrite Growth by Forming a Thin, Less-Resistive 
Solid Electrolyte Interphase. ACS Energy Letters, 2016. 1(2): p. 414-419.

101. Janek, J. and W.G. Zeier, A Solid Future for Battery Development. Nature Energy, 2016. 1: p. 16141.

102. Kummer, J.T., A. Arbor, and N. Weber, Thermo-Electric Generator. US patent 3, 1969. 458: p. 356.

103. Manthiram, A., X. Yu, and S. Wang, Lithium battery Chemistries Enabled by Solid-State Electrolytes. Nature Reviews Materials, 2017. 2(4): p. 16103.

104. Hu, Y.S., Batteries: Getting Solid. Nature Energy, 2016. 1: p. 16042.

105. Quartarone, E. and P. Mustarelli, Electrolytes for Solid-State Lithium Rechargeable Batteries: Recent Advances and Perspectives. Chemical Society Reviews, 2011. 40: p. 2525-2540.

106. J.B.Bates, et al., Electrical Properties of Amorphous Lithium Electrolyte Thin Films. Solid State Ionics, 1992. 53-56: p. 647-654.

107. Feng, J.K., et al., All Solid State Lithium Ion Rechargeable Batteries Using NASICON Structured Electrolyte. Materials Technology, 2013. 28(5): p. 276-279.

108. Ihlefeld, J.F., et al., Fast Lithium-Ion Conducting Thin-Film Electrolytes Integrated Directly on Flexible Substrates for High-Power Solid-State Batteries. Advanced Materials, 2011. 23(47): p. 5663-5667.

109. Kennedy, J.H., et al., Preparation and Conductivity Measurements of SiS $\mathrm{SL}_{2} \mathrm{~S}$ Glasses Doped with $\mathrm{LiBr}$ and $\mathrm{LiCl}$. Solid State Ionics, 1986. 18-19: p. 368-371.

110. Cussen, E.J., The Structure of Lithium Garnets: Cation Disorder and Clustering in a New Family of Fast $\mathrm{Li}^{+}$Conductors. Chemical Communications, 2006: p. 412-413.

111. Kamaya, N., et al., A Lithium Superionic Conductor. Nature Materials, 2011. 10(9): p. 682-686. 112. Shin, B.R., et al., Comparative Study of TiS $2 / L i$-In All-Solid-State Lithium Batteries Using Glass-Ceramic $\mathrm{Li}_{3} \mathrm{PS}_{4}$ and $\mathrm{Li}_{10} \mathrm{GeP}_{2} \mathrm{~S}_{12}$ Solid Electrolytes. Electrochimica Acta, 2014. 146: p. 395-402.

113. Chen, R., et al., The Pursuit of Solidstate Electrolytes for Lithium Batteries: From Comprehensive Insight to Emerging Horizons. Materials Horizons, 2016. 3: p. 487-516.

114. Inaguma, Y., et al., High Ionic Conductivity in Lithium Lanthanum Titanate. Solid State Communications, 1993. 86: p. 689-693.

115. Thangadurai, V. and W. Weppner, Recent Progress in Solid Oxide and Lithium Ion Conducting Electrolytes Research. Ionics, 2006. 12: p. 81-92.

116. Jie, F., Superionic Conductivity of Glass-Ceramics in the System $\mathrm{Li}_{2} \mathrm{O}-\mathrm{Al}_{2} \mathrm{O}_{3}-\mathrm{TiO}_{2}-\mathrm{P}_{2} \mathrm{O}_{5}$. Solid State Ionics, 1997. 96: p. 195-200.

117. Kim, H.S., et al., Characterization of Sputter-Deposited $\mathrm{LiCoO}_{2}$ Thin Film Grown on NASICON-type Electrolyte for Application in All-Solid-State Rechargeable Lithium Battery. ACS Applied Materials \& Interfaces, 2017. 9(19): p. 16063-16070.

118. Bernuy-Lopez, C., et al., Atmosphere Controlled Processing of Ga-Substituted Garnets for High Li-Ion Conductivity Ceramics. Chemistry of Materials, 2014. 26(12): p. 3610-3617.

119. Du, F.M., et al., All Solid State Lithium Batteries Based on Lamellar Garnet-Type Ceramic Electrolytes. Journal of Power Sources, 2015. 300: p. 24-28.

120. Kim, J.G., et al., A Review of Lithium and Non-Lithium Based Solid State Batteries. Journal of Power Sources, 2015. 282: p. 299-322.

121. Han, X., et al., Negating Interfacial Impedance in Garnet-Based Solid-State Li Metal Batteries. Nature Materials, 2017. 16(5): p. 572-579.

122. Cheng, L., et al., The Origin of High Electrolyte-Electrode Interfacial Resistances in Lithium 
Cells Containing Garnet Type Solid Electrolytes. Physical Chemistry Chemical Physics, 2014. 16(34): p. 18294-18300.

123. Kelly, I.E., J.R. Owen, and B.C.H. Steele, Poly(ethylene oxide) Electrolytes for Operation at near Room Temperature. Journal of Power Sources, 1985. 14: p. 13-21.

124. Sumathipala, H.H., et al., High Performance PEO-Based Polymer Electrolytes and their Application in Rechargeable Lithium Polymer Batteries. Ionics, 2007. 13(5): p. 281-286.

125. Xue, Z.G., D. He, and X.L. Xie, Poly(ethylene oxide)-Based Electrolytes for Lithium-Ion Batteries. Journal of Materials Chemistry A, 2015. 3(38): p. 19218-19253.

126. Kim, D.G., et al., Preparation of Solid-State Composite Electrolytes Based on Organic/Inorganic Hybrid Star-Shaped Polymer and PEG-Functionalized POSS for All-Solid-State Lithium Battery Applications. Polymer, 2013. 54(21): p. 5812-5820.

127. Kim, S.K., et al., Organic/Inorganic Hybrid Block Copolymer Electrolytes with Nanoscale Ion-Conducting Channels for Lithium Ion Batteries. Macromolecules, 2012. 45(23): p. 9347-9356.

128. Michael, M.S. and S.R.S. Prabaharan, Rechargeable Lithium Battery Employing a New Ambient Temperature Hybrid Polymer Electrolyte Based on PVK plus PVdF-HFP (copolymer). Journal of Power Sources, 2004. 136(2): p. 408-415.

129. Shi, J., Y.F. Yang, and H.X. Shao, Co-polymerization and Blending Based PEO/PMMA/P(VDF-HFP) Gel Polymer Electrolyte for Rechargeable Lithium Metal Batteries. Journal of Membrane Science, 2018. 547: p. 1-10.

130. Chen, L., Y. Liu, and L.-Z. Fan, Enhanced Interface Stability of Polymer Electrolytes Using Organic Cage-Type Cucurbit[6]uril for Lithium Metal Batteries. Journal of the Electrochemical Society, 2017. 164: p. A1834-A1840.

131. Lu, Q., et al., Dendrite-Free, High-Rate, Long-Life Lithium Metal Batteries with a 3D Cross-Linked Network Polymer Electrolyte. Advanced Materials, 2017. 29(13).

132. Goujon, L.J., et al., Flexible Solid Polymer Electrolytes Based on Nitrile Butadiene Rubber/Poly(ethylene oxide) Interpenetrating Polymer Networks Containing Either LiTFSI or EMITFSI. Macromolecules, 2011. 44(24): p. 9683-9691.

133. Lin, Z., X. Guo, and H. Yu, Amorphous Modified Silyl-Terminated 3D Polymer Electrolyte for High-Performance Lithium Metal Battery. Nano Energy, 2017. 41: p. 646-653.

134. Chai, J., et al., In Situ Generation of Poly (Vinylene Carbonate) Based Solid Electrolyte with Interfacial Stability for $\mathrm{LiCoO}_{2}$ Lithium Batteries. Advanced Science (Weinh), 2017. 4(2): p. 1600377.

135. Shim, J., et al., All-Solid-State Lithium Metal Battery with Solid Polymer Electrolytes Based on Polysiloxane Crosslinked by Modified Natural Gallic Acid. Polymer, 2017. 122: p. 222-231.

136. Zhou, D., et al., Investigation of Cyano Resin-Based Gel Polymer Electrolyte: In Situ Gelation Mechanism and Electrode-Electrolyte Interfacial Fabrication in Lithium-Ion Battery. Journal of Materials Chemistry A, 2014. 2(47): p. 20059-20066.

137. Liu, X., et al., An Interpenetrating Network Poly(diethylene glycol carbonate)-Based Polymer Electrolyte for Solid State Lithium Batteries. Journal of Materials Chemistry A, 2017. 5(22): p. 11124-11130.

138. Zhao, C.Z., et al., An Anion-Immobilized Composite Electrolyte for Dendrite-Free Lithium Metal Anodes. Proceedings of the National Academy of Sciences of the United States of America, 2017. 114(42): p. 11069-11074.

139. Zhou, W., et al., Plating a Dendrite-Free Lithium Anode with a Polymer/Ceramic/Polymer Sandwich Electrolyte. Journal of America Chemistry Society, 2016. 138(30): p. 9385-9388. 
140. Wang, C., et al., Suppression of Lithium Dendrite Formation by Using LAGP-PEO (LiTFSI) Composite Solid Electrolyte and Lithium Metal Anode Modified by PEO (LiTFSI) in All-Solid-State Lithium Batteries. ACS Applied Materials \& Interfaces, 2017. 9(15): p. 13694-13702.

141. Hu, J., J. Tian, and C. Li, Nanostructured Carbon Nitride Polymer-Reinforced Electrolyte to Enable Dendrite-Suppressed Lithium Metal Batteries. ACS Applied Materials \& Interfaces, 2017. 9(13): p. 11615-11625.

142. Fu, K.K., et al., Flexible, Solid-State, Ion-Conducting Membrane with 3D Garnet Nanofiber Networks for Lithium Batteries. Proceedings of the National Academy of Sciences of the United States of America, 2016. 113: p. 7094-7099.

143. Shim, J., et al., 2D Boron Nitride Nanoflakes as a Multifunctional Additive in Gel Polymer Electrolytes for Safe, Long Cycle Life and High Rate Lithium Metal Batteries. Energy \& Environmental Science, 2017. 10(9): p. 1911-1916.

144. Li, Y., et al., Hybrid Polymer/Garnet Electrolyte with a Small Interfacial Resistance for Lithium-Ion Batteries. Angewandte Chemie International Edition in English, 2017. 56(3): p. 753-756.

145. Gurevitch, I., et al., Nanocomposites of Titanium Dioxide and Polystyrene-Poly(ethylene oxide) Block Copolymer as Solid-State Electrolytes for Lithium Metal Batteries. Journal of the Electrochemical Society, 2013. 160(9): p. A1611-A1617.

146. Amiri, H. and M. Mohsennia, Impedance Study of PVA/PEG/LiClO $/ \mathrm{TiO}_{2}$ Nanocomposite Solid Polymer Blend Electrolyte. Journal of Materials Science: Materials in Electronics, 2017. 28(6): p. 4586-4592.

147. Zhang, J.X., et al., Flexible and Ion-Conducting Membrane Electrolytes for Solid-State Lithium Batteries: Dispersion of Garnet Nanoparticles in Insulating Polyethylene Oxide. Nano Energy, 2016. 28: p. 447-454.

148. Wang, Y.J., Y. Pan, and D. Kim, Conductivity Studies on Ceramic $\mathrm{Li}_{1.3} \mathrm{Al}_{0.3} \mathrm{Ti}_{1.7}\left(\mathrm{PO}_{4}\right)_{(3)}$-Filled PEO-Based Solid Composite Polymer Electrolytes. Journal of Power Sources, 2006. 159(1): p. 690-701.

149. Zhou, W.D., H.C. Gao, and J.B. Goodenough, Low-Cost Hollow Mesoporous Polymer Spheres and All-Solid-State Lithium, Sodium Batteries. Advanced Energy Materials, 2016. 6(1).

150. Wang, W., et al., Lithium Ion Conducting Poly(ethylene oxide)-Based Solid Electrolytes Containing Active or Passive Ceramic Nanoparticles. The Journal of Physical Chemistry C, 2017. 121(5): p. 2563-2573.

151. Liu, W., et al., Ionic Conductivity Enhancement of Polymer Electrolytes with Ceramic Nanowire Fillers. Nano Letters, 2015. 15(4): p. 2740-2745.

152. Zhu, J., et al., A Stable Organic-Inorganic Hybrid Layer Protected Lithium Metal Anode for Long-Cycle Lithium-Oxygen Batteries. Journal of Power Sources, 2017. 366: p. 265-269.

153. Li, N.W., et al., Smart Solid Electrolyte Interphase Layer for Long Life Lithium Metal Anodes. Angewandte Chemie International Edition in English, 2017.

154. Gauthier, M., et al., Electrode-Electrolyte Interface in Li-Ion Batteries: Current Understanding and New Insights. Journal of Physical Chemistry Letters, 2015. 6(22): p. 4653-4672.

155. Wu, M., et al., Electrochemical Behaviors of a Li $i_{3} N$ Modified Li Metal Electrode in Secondary Lithium Batteries. Journal of Power Sources, 2011. 196(19): p. 8091-8097.

156. Guo, Y., H. Li, and T. Zhai, Reviving Lithium-Metal Anodes for Next-Generation High-Energy Batteries. Advanced Materials, 2017. 29(29).

157. Wang, L., et al., Li metal Coated with Amorphous $\mathrm{Li}_{3} \mathrm{PO}_{4}$ via Magnetron Sputtering for Stable 
and Long-Cycle Life Lithium Metal Batteries. Journal of Power Sources, 2017. 342: p. 175-182.

158. Fan, L., et al., Regulating Li Deposition at Artificial Solid Electrolyte Interphases. Journal of Materials Chemistry A, 2017. 5(7): p. 3483-3492.

159. A.C. Kozen, et al., Next-Generation Lithium Metal Anode Engineering via Atomic Layer Deposition. ACS Nano, 2015. 9: p. 5884-5892.

160. Kazyak, E., K.N. Wood, and N.P. Dasgupta, Improved Cycle Life and Stability of Lithium Metal Anodes through Ultrathin Atomic Layer Deposition Surface Treatments. Chemistry of Materials, 2015. 27(18): p. 6457-6462.

161. Kim, M.S., et al., Designing Solid-Electrolyte Interphases for Lithium Sulfur Electrodes Using Ionic Shields. Nano Energy, 2017. 41: p. 573-582.

162. Peng, Z., et al., Stabilizing Li/Electrolyte Interface with a Transplantable Protective Layer Based on Nanoscale LiF Domains. Nano Energy, 2017. 39: p. 662-672.

163. Lu, Y., et al., Pre-Modified $\mathrm{Li}_{3} \mathrm{PS}_{4}$ Based Interphase for Lithium Anode towards High-Performance Li-S Battery. Energy Storage Materials, 2018. 11: p. 16-23.

164. Kim, M.S., et al., Fabricating Multifunctional Nanoparticle Membranes by a Fast Layer-by-Layer Langmuir-Blodgett Process: Application in Lithium-Sulfur Batteries. Journal of Materials Chemistry A, 2016. 4(38): p. 14709-14719.

165. Huang, J.Q., et al., Ionic Shield for Polysulfides towards Highly-Stable Lithium-Sulfur Batteries. Energy Environmental Science, 2014. 7(1): p. 347-353.

166. Wang, L., et al., Long Lifespan Lithium Metal Anodes Enabled by $\mathrm{Al}_{2} \mathrm{O}_{3}$ Sputter Coating. Energy Storage Materials, 2018. 10: p. 16-23.

167. Ye, H., et al., Synergism of Al-Containing Solid Electrolyte Interphase Layer and Al-Based Colloidal Particles for Stable Lithium Anode. Nano Energy, 2017. 36: p. 411-417.

168. Belov, D.G., et al., Lithium Surface Protection by Polyacetylene In Situ Polymerization. Synthetic Metals, 2006. 156(9-10): p. 745-751.

169. Choi, S.M., et al., Cycling Characteristics of Lithium Metal Batteries Assembled with a Surface Modified Lithium Electrode. Journal of Power Sources, 2013. 244: p. 363-368.

170. Gao, Y., et al., Interfacial Chemistry Regulation via a Skin-Grafting Strategy Enables High-Performance Lithium-Metal Batteries. Journal of America Chemistry Society, 2017. 139(43): p. $15288-15291$.

171. Kozen, A.C., et al., Stabilization of Lithium Metal Anodes by Hybrid Artificial Solid Electrolyte Interphase. Chemistry of Materials, 2017. 29(15): p. 6298-6307.

172. Yang, C., et al., Protected Lithium-Metal Anodes in Batteries: From Liquid to Solid. Advanced Materials, 2017. 29(36).

173. Ryou, M.H., et al., Excellent Cycle Life of Lithium-Metal Anodes in Lithium-Ion Batteries with Mussel-Inspired Polydopamine-Coated Separators. Advanced Energy Materials, 2012. 2(6): p. 645-650.

174. Park, K., et al., New Battery Strategies with a Polymer $/ \mathrm{Al}_{2} \mathrm{O}_{3}$ Separator. Journal of Power Sources, 2014. 263: p. 52-58.

175. Ye, Y., et al., A Modularly-Assembled Interlayer to Entrap Polysulfides and Protect Lithium Metal Anode for High Areal Capacity Lithium-Sulfur Batteries. Energy Storage Materials, 2017. 9: p. 126-133.

176. Sacci, R.L., et al., Nanoscale Imaging of Fundamental Li Battery Chemistry: Solid-Electrolyte Interphase Formation and Preferential Growth of Lithium Metal Nanoclusters. Nano Letters, 2015. 15(3): p. 2011-2018. 
177. Zhang, R., et al., Advanced Micro/Nanostructures for Lithium Metal Anodes. Advanced Science (Weinh), 2017. 4(3): p. 1600445.

178. Zhang, R., et al., Lithiophilic Sites in Doped Graphene Guide Uniform Lithium Nucleation for Dendrite-Free Lithium Metal Anodes. Angewandte Chemie International Edition in English, 2017. 56(27): p. 7764-7768.

179. Lu, Z., et al., Improving Li Anode Performance by a Porous 3D Carbon Paper Host with Plasma Assisted Sponge Carbon Coating. Energy Storage Materials, 2017. 11: p. 47-56.

180. Lang, J., et al., Surface Graphited Carbon Scaffold Enables Simple and Scalable Fabrication of 3D Composite Lithium Metal Anode. Journal of Materials Chemistry A, 2017. 5(36): p. 19168-19174.

181. Lin, D.C., et al., Layered Reduced Graphene Oxide with Nanoscale Interlayer Gaps as a Stable Host for Lithium Metal Anodes. Nature Nanotechnology, 2016. 11(7): p. 626-633.

182. Wang, A., et al., Bending-Tolerant Anodes for Lithium-Metal Batteries. Advanced Materials, 2017. 30(1): p. 1703891.

183. Liu, S., et al., Crumpled Graphene Balls Stabilized Dendrite-free Lithium Metal Anodes. Joule, 2017. 1(17): p. 184-193.

184. Yan, K., et al., Three-Dimensional Pie-like Current Collectors for Dendrite-Free Lithium Metal Anodes. Energy Storage Materials, 2018. 11: p. 127-133.

185. Liang, Z., et al., Composite Lithium Metal Anode by Melt Infusion of Lithium into a 3D Conducting Scaffold with Lithiophilic Coating. Proceedings of the National Academy of Sciences of the United States of America, 2016. 113(11): p. 2862-2867.

186. Wang, S.H., et al., Stable Li Metal Anodes via Regulating Lithium Plating/Stripping in Vertically Aligned Microchannels. Advanced Materials, 2017. 29(40): p. 1703729.

187. Lin, C.F., et al., ALD Protection of Li-Metal Anode Surfaces-Quantifying and Preventing Chemical and Electrochemical Corrosion in Organic Solvent. Advanced Materials Interfaces, 2016. 3(21): p. 1600426.

188. Li, Q., S. Zhu, and Y. Lu, 3D Porous Cu Current Collector/Li-Metal Composite Anode for Stable Lithium-Metal Batteries. Advanced Functional Materials, 2017. 27(18): p. 1606422.

189. Xu, Y., et al., Honeycomb-like Porous 3D Nickel Electrodeposition for Stable Li and Na Metal Anodes. Energy Storage Materials, 2018. 12: p. 69-78.

190. Zhang, Y., et al., High-Capacity, Low-Tortuosity, and Channel-Guided Lithium Metal Anode. Proceedings of the National Academy of Sciences of the United States of America, 2017. 114: p. 3584-3589.

191. Lu, L.L., et al., Lithiophilic Cu-Ni Core-Shell Nanowire Network as a Stable Host for Improving Lithium Anode Performance. Energy Storage Materials, 2017. 9: p. 31-38.

192. Lin, D., et al., Three-Dimensional Stable Lithium Metal Anode with Nanoscale Lithium Islands Embedded in Ionically Conductive Solid Matrix. Proceedings of the National Academy of Sciences of the United States of America, 2017. 114(18): p. 4613-4618.

193. Wang, H., et al., Ultrahigh-Current Density Anodes with Interconnected Li Metal Reservoir through Overlithiation of Mesoporous AlF 3 Framework. Science Advances, 2017. 3: p. e1701301.

194. Wang, L., et al., ZnO/Carbon Framework Derived from Metal-Organic Frameworks as a Stable Host for Lithium Metal Anodes. Energy Storage Materials, 2018. 11: p. 191-196.

195. Chi, S.S., et al., Prestoring Lithium into Stable 3D Nickel Foam Host as Dendrite-Free Lithium Metal Anode. Advanced Functional Materials, 2017. 27(24): p. 1700348.

196. Yan, K., et al., Selective Deposition and Stable Encapsulation of Lithium through 
Heterogeneous Seeded Growth. Nature Energy, 2016. 1.

197. Yang, C., et al., Ultrafine Silver Nanoparticles for Seeded Lithium Deposition toward Stable Lithium Metal Anode. Advanced Materials, 2017. 29(38): p. 1702714.

198. Cheng, X.B., et al., Dendrite-Free Lithium Deposition Induced by Uniformly Distributed Lithium Ions for Efficient Lithium Metal Batteries. Advanced Materials, 2016. 28(15): p. 2888-2895.

199. Lee, Y.G., et al., Dendrite-Free Lithium Deposition for Lithium Metal Anodes with Interconnected Microsphere Protection. Chemistry of Materials, 2017. 29(14): p. 5906-5914.

200. Ramesh, S., et al., FTIR Studies of PVC/PMMA Blend Based Polymer Electrolytes. Spectrochimica Acta Part a-Molecular and Biomolecular Spectroscopy, 2007. 66(4-5): p. 1237-1242.

201. Liang, Z., et al., Polymer Nanofiber-Guided Uniform Lithium Deposition for Battery Electrodes. Nano Letters, 2015. 15(5): p. 2910-2916.

202. Matsuda, S., et al., Insulative Microfiber 3D Matrix as a Host Material Minimizing Volume Change of the Anode of Li Metal Batteries. ACS Energy Letters, 2017. 2(4): p. 924-929.

203. Lang, J.L., et al., Uniform Lithium Deposition Induced by Polyacrylonitrile Submicron Fiber Array for Stable Lithium Metal Anode. Acs Applied Materials \& Interfaces, 2017. 9(12): p. 10360-10365. 